STRESS, COGNITION AND FUNCTIONAL CONNECTIVITY IN THE HEALTHY HUMAN BRAIN

A Dissertation
presented to
the Faculty of the Graduate School
at the University of Missouri-Columbia
In Partial Fulfillment
of the Requirements for the Degree
Doctor of Philosophy
Dr. David Q. Beversdorf, Dissertation Supervisor
NEETU NAIR

DECEMBER 2018 
The undersigned, appointed by the dean of the Graduate School, have examined the dissertation entitled

STRESS, COGNITION AND FUNCTIONAL CONNECTIVITY IN THE HEALTHY HUMAN BRAIN

presented by Neetu Nair, a candidate for the degree of Doctor of Philosophy in neuroscience, and hereby certify that, in their opinion, it is worthy of acceptance.

David Q. Beversdorf, MD

Carmen Cirstea, MD, $\mathrm{PhD}$

Jeffrey D. Johnson, PhD

Joel Shenker, MD, $\mathrm{PhD}$ 
To my husband, whose love and support has propelled me through the toughest phases of this journey and life in general.

To my daughter, for being the most perfect child any mother could ever get. I love you.

To my mother, for who I am and who I will become.

To my family, for their unrelenting faith in me always.

To all the participants, without whose patience and cooperation, these studies would not have been possible. 


\section{ACKNOWLEDGEMENTS}

I would like to sincerely thank Dr. David Beversdorf for his support and guidance throughout my time in the lab. I would also like to express my heartfelt gratitude to all my committee members - Dr. Jeffrey Johnson, Dr. Carmen Cirstea and Dr. Joel Shenker and past mentors - Dr. Stanislow Majewski, Dr. Julie Brefcynzki-Lewis, Dr. Manish Monga and Dr. Marc Haut for their valuable guidance. I would also like to express my gratitude to Dr. John Lauriello and the Department of Psychiatry at the University of Missouri Hospital for the kind support during my early days of graduate school. I am extremely thankful to Dr. Huiling Peng and other faculty and staff at the Brain Imaging Center for being so kind, patient and instrumental in my learning process. Throughout my time in grad school, I have been truly blessed to have some amazing lab mates and coworkers who have constantly encouraged, guided and supported me through all the ups and downs, especially Allison Halt, Brad Ferguson, Briana Michelle, Hannah Rogers, John Hegarty, Rachel Zamzow, and Taeseon Woo. I would like to thank each of them from the bottom of my heart. I could not have accomplished this without each of you. I would like to acknowledge and appreciate all my coworkers at the Thompson Center for Autism and Neurodevelopmental Disorders. Everyone, from the physicians to the office staff, has been absolutely wonderful and I really enjoyed my time working with them. A special thank you to Bridget Lolli, RN for being the amazing person that she is and an absolute pleasure to work with. I also owe gratitude to the wonderful undergraduate research assistants who have worked with me over the years. Working with them has been an absolutely enriching experience for me as I hope it has been for them as well. Last but not the least, a special thank you to my wonderful 
family and friends who have constantly rooted for me and been my biggest support system throughout this journey. 


\section{TABLE OF CONTENTS}

ACKNOWLEDGEMENTS ................................................ ii

LIST OF FIGURES............................................... vi

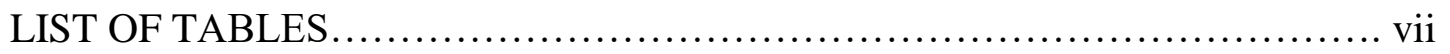

LIST OF ABBREVIATIONS.......................................... viii

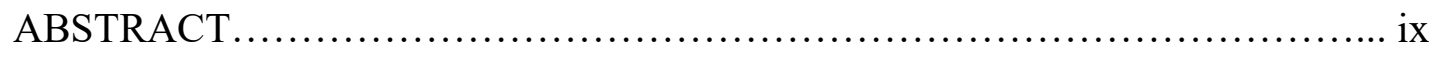

Chapter

I. INTRODUCTION

Stress - General Considerations........................... 1

Stress, brain and cognition.................................. 3

II. EFFECTS OF STRESS ON FUNCTIONAL CONNECTIVITY DURING

VERBAL PROCESSING

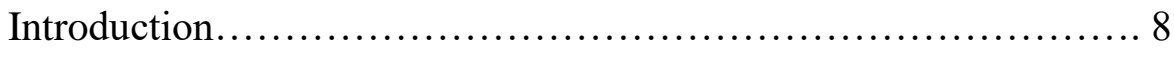

Materials and Methods........................................ 14

Results.............................................. 21

Discussion................................................... 30

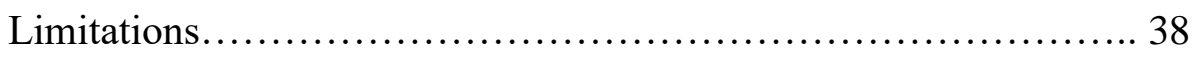

Summary.............................................. 39

III. EXPLORING FUNCTIONAL CONNECTIVITY CORRELATES OF VERBAL PROBLEM SOLVING UNDER STRESS

Introduction............................................... 40

Materials and Methods......................................45

Results................................................. 52 
Discussion............................................ 60

Limitations............................................... 64

Summary ............................................ 65

IV. AMYGDALA-FUSIFORM FUNCTIONAL CONNECTIVITY RESPONSE TO EMOTIONAL FACES UNDER STRESS

Introduction........................................... 66

Materials and Methods......................................... 69

Results.............................................. 75

Discussion............................................. 80

Limitations............................................ 82

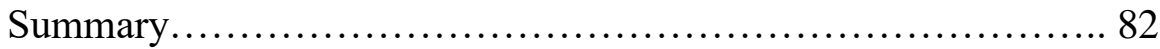

V. CONCLUSIONS AND FUTURE DIRECTIONS .................84

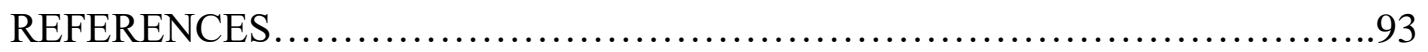

VITA............................................................... 125 


\section{LIST OF FIGURES}

Figure

Page

2.1 Graphic showing stimulus protocol design for letter and category

fluency tasks................................................. 18

Regions of interest............................................. 20

2.3 Group activation maps.......................................... 23

2.4 Functional connectivity strength during letters fluency task under stress vs. no stress.................................................. 26

2.5 Functional connectivity strength during category fluency task under stress vs. no stress............................................... 28

3.1 Stimulus protocol for creativity tasks................................ 48

Regions of interest............................................... 50

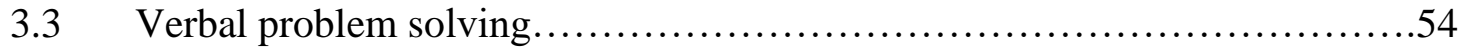

$3.4 \quad$ Group activation maps........................................... 54

3.5 Regional activation differences in the right superior temporal gyrus during verbal problem solving under stress vs. no stress...................56

3.6 Functional connectivity changes between the left inferior frontal gyrus and the left middle temporal gyrus during verbal problem solving under stress vs. no stress ............................................58

4.1 Sample image for faces task .................................... 71

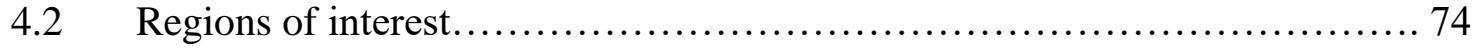

$4.3 \quad$ Group activation maps.............................................. 76

4.4 Differences in mean activation in the left amygdala while viewing emotional faces under stress and no stress. 


\section{LIST OF TABLES}

Table

Page

2.1 Participant demographics for verbal fluency study............... 22

3.1 Participant demographics for verbal problem solving study..........52

4.1 Participant demographics for emotional face viewing study..........75 


\section{LIST OF ABBREVIATIONS}

4D

AC-PC

BET

BOLD

COMT

CRA

DNA

EEG

ELS

EPI

FC

FEAT

FLIRT

$f$ MRI

FOV

FSL

FWHM

HPA

IAPS

IQ

MANCOVA

MANOVA

MCFLIRT

MIST

MPRAGE

MRI

NAART

ROI

SD

SLC6A4

SNP

STAI

TE

TR

TSST
Four dimensional

Anterior commissure - Posterior commissure

Brain extraction Tool

Blood Oxygenation Level Dependent

Catechol-O-methyltransferase

Compound Remote Associates

Deoxyribonucleic acid

Electroencephalography

Early Life Stress

Echo Planar Imaging

Functional Connectivity

fMRI Expert Analysis Tool

FMRIB's Linear Registration Tool

Functional Magnetic Resonance Imaging

Field of view

FMRIB Software Library

Full-width Half Maximum

Hypothalamic - pituitary - adrenal

International Affective Picture System

Intelligence Quotient

Multivariate Analysis of Covariance

Multivariate Analysis of Variance

Motion Correction with FMRIB's Linear Registration Tool

Montreal Imaging Stress Test

Magnetization Prepared Rapid Acquisition Gradient Echo

Magnetic Resonance Imaging

North American Adult Reading Test

Region of Interest

Standard deviation

Sodium dependent serotonin transporter and Solute carrier family 6 member 4

Single nucleotide polymorphisms

State-Trait Anxiety Inventory

Time to echo

Time to repeat

Trier Social Stress Test 


\title{
STRESS, COGNITION AND FUNCTIONAL CONNECTIVITY IN THE
}

\section{HEALTHY HUMAN BRAIN}

\author{
Neetu Nair \\ Dr. David Q. Beversdorf, Dissertation Supervisor
}

\begin{abstract}
Stress is known to negatively impact physiological and psychological processes in humans. While chronic exposure to stress has been implicated in the pathology of numerous physiological and neuropsychological disorders, acute stress exposure has also been shown to impair cognitive task performance. Since the brain is the primary organ involved in detecting and responding to stressors, neuroimaging techniques have been used to explore the structural and functional neural correlates of stress. The studies presented here attempted to examine how acute stress might affect the temporally correlated activation of multiple brain regions, also known as functional connectivity, while performing cognitive tasks. Of additional interest was the role of gender and presence of the short allele polymorphism on the promoter region of the serotonin transporter gene, both factors known to influence stress susceptibility, in determining effects of stress on functional connectivity during cognitive task performance. Forty-five participants with no history of neurological or psychiatric disorders were recruited to participate in the study and underwent two sessions of functional magnetic resonance imaging - one session involved exposure to the Montreal Imaging Stress Test (MIST) to induce stress and the other session had control tasks that did not induce stress. The participants were blinded to the stress induction. Following exposure to the stress or no stress control task, the participants performed verbal fluency tasks, verbal problem-solving tasks and the emotional faces task. A priori regions of interest were defined for each of the tasks and functional connectivity differences between the a
\end{abstract}


priori regions of interest under stress and no stress were examined. Additionally, the influence of gender and genotype were also examined. Results reveal specific gender and genotype-based differences in regional functional connectivity under stress and no stress during the cognitive tasks even in the absence of differences in overall task performance. The results begin to elucidate the specific neural underpinnings of stress susceptibility in healthy individuals. The implications of these results in better understanding the neural correlates of stress related cognitive impairments, and may ultimately be helpful for neuropsychiatric disorders such as anxiety, depression and posttraumatic stress disorders and may help us move towards developing targeted neuropharmacological therapeutic interventions are discussed. 


\section{CHAPTER ONE:}

\section{INTRODUCTION}

Stress has invariably become a part of day-to-day life in the modern world and has led to the increased incidence of stress related disorders. Consequently, the economic burden associated with stress related disabilities is on the rise as well (Kalia, 2002). Not surprisingly, increased levels of stress have been implicated in the development of hypertension, cardiovascular disease, anxiety, depression and progression of conditions such as HIV/AIDS and cancer as well (Cohen, Janicki-deverts, \& Miller, 2007). While the perception of a situation to be stressful and the resilience in adapting to a stressful situation varies on an individual basis, research has consistently shown that exposure to even short duration stressors produces a myriad of psychological and physiological changes in even healthy individuals. Understanding how stress affects the human body has, therefore, gained a lot of attention over the last few decades.

\section{Stress - General Considerations}

For the purpose of the discussions here, stress will been defined as a condition wherein external factors disrupt the dynamic equilibrium that an organism maintains (Chrousos, 2009), such as situations where the task demands are perceived to be in excess of ability (Cohen et al., 2007). Consequently, the condition gives rise to a perception of threat towards the organism's well-being and/or safety, initiating a fight-or-flight response essential towards ensuring survival at the level of an individual organism as well as the 
species as a whole. The stress response is evolutionarily meant to promote survival in unfavorable conditions and exerts an Inverted-U shaped effect, such that there is a range of stress under which optimal performance is elicited. Hypo reactivity and hyper reactivity to stress, which fall on either ends of the Inverted-U, both elicit unfavorable responses (Chrousos, 2009). Hypo reactivity to stress would result in lack of inhibition while approaching a potential dangerous situation or threat while hyper reactivity would result in the inability to overcome fear, avoidance of even non-harmful situations and consequently inability to form meaningful social relationships essential to survival.

Ensuring that the stress response remains in the optimal functioning range requires the synchronous activity of numerous systems within the body. The locus coeruleusnorepinephrine system, for example, produces immediate but transient response to stressors by releasing epinephrine and norepinephrine to regulate sympathetic activity (Lucassen et al., 2014). Dysregulated activity in the locus coeruleus has been implicated in various stress related disorders such as phobias, panic disorder and generalized anxiety disorder (Bremner, Krystal, Southwick, \& Charney, 2006). Within minutes of exposure to an acute stressor, hypothalamic - pituitary - adrenal (HPA) axis activity leads to production of cortisol (Lucassen et al., 2014). Since cortisol receptors are present throughout the body, the release of cortisol is accompanied by widespread effects on the brain, heart, immune system and even metabolic system. Evidence from studies performing pharmacological modulation of the locus coeruleus-norepinephrine system by using beta adrenergic antagonists have shown corresponding changes in HPA axis 
activity providing evidence for their combined activity during stress response (Andrews \& Pruessner, 2013).

\section{Stress, brain and cognition}

Activation of the stress response systems as mentioned above, results in increased cardiovascular tone, a shifting of resources from tasks that are non-essential to immediate survival, such as reproduction, digestion and tissue repair, to more essential domains such as muscle contractility, hypervigilance and narrowed, focused attention on the immediate threat. From an evolutionary standpoint, this system is well equipped to handle possible threats such as the presence of predators (Nesse, Bhatnagar, \& Ellis, 2016). However, in the present day, triggers for the stress response are much more diversified. For example, test taking and public speaking are common stressful situations in the modern world. Neither of these pose an immediate threat to survival but do place considerable demands on the cognitive resources of an individual, including his/her ability to retrieve, combine and assimilate information and present it in a useable context. Stress induced hypervigilance and narrowed, focused attention, therefore, may actually be detrimental towards performance on such cognitive tasks requiring flexible thinking.

The brain is the obligate primary organ involved in the detection of and response to stressors (Mcewen, 2009). As such, not only does the brain initiate production of stress hormones, but structure and function of regions within the brain are affected by stress hormones, including norepinephrine and cortisol. The amygdala, hippocampus and 
prefrontal cortex in humans are known to undergo specific structural alterations such as reduction in volume associated with increases in HPA axis activity under stress (McEwen, Nasca, \& Gray, 2016). Further, extensive deactivation of the limbic system in response to high levels of cortisol following stress exposure have also been noted (J. C. Pruessner et al., 2008). Liston, Mcewen, \& Casey, 2009 have reported on how acute stress exposure transiently altered activity within the prefrontal regions of the brain and Hermans, Marle, et al., 2011 showed that noradrenergic activity associated with stress exposure resulted in increased coupling between brain regions involved in "autonomicneuroendocrine control and vigilant attentional reorienting." In general, the consensus is that under stress, the brain shifts from a state of voluntary higher cognitive control to involuntary, survival instinct mode. Thus, stress is associated with hypervigilance and narrowed attention. As a result, under conditions of stress, performance on cognitive tasks that require flexible access to multiple, remote networks may be impaired. Research evidence in support of this argument comes from studies showing that under stress, performance on cognitive flexibility tasks such as anagrams and the Compound Remote Associates Task are impaired and this effect is rescued by administration of a betaadrenergic antagonist such as propranolol (J. K. Alexander, Hillier, Smith, Tivarus, \& Beversdorf, 2007). Additionally, the beneficial of beta adrenergic antagonists were observed only in conditions where either the problems presented were difficult to begin with (Campbell, Tivarus, Hillier, \& Beversdorf, 2008), there was increased noradrenergic tone associated with conditions such as acute cocaine withdrawal (Kelley, Yeager, Pepper, Bornstein, \& Beversdorf, 2007) or when flexible access to remote networks was impaired due to anatomical constraints (Beversdorf et al., 2007). 
Apart from anatomical and functional activation differences in brain regions following exposure to stress, neuroimaging studies have looked at the temporally correlated activity between brain regions as well, a phenomenon known as functional connectivity. Studies have so far looked at changes in resting state functional connectivity in the brain following exposure to stress (Hermans, Henckens, Joëls, \& Fernández, 2014; Soares et al., 2013; Vaisvaser et al., 2013; H. J F van Marle, Hermans, Qin, \& Fernández, 2010; Veer et al., 2011) and at functional connectivity changes under stress during cognitive tasks that do not necessarily require use flexible access to remote resources (Ossewaarde et al., 2011; Shaozheng Qin, Hermans, van Marle, Luo, \& Fernández, 2009). As mentioned before, cognitive tasks require flexible access to multiple, possibly remote functional connections, an ability specifically compromised under conditions of stress. Therefore, using neuroimaging to understand the mechanism by which cognitive flexibility is impaired under stress is of interest. Functional connectivity changes may then be explored as potential biomarkers for stress response in future studies looking at interventions to minimize the negative effects of stress, especially in the cognitive flexibility domain.

The first two studies presented here explored the effects of stress on functional connectivity between brain regions while performing cognitive tasks requiring flexible access to multiple brain networks. The third study, in an attempt to better understand the neural underpinnings of the stress response, explored whether stress upregulated the 
functional connectivity between the amygdala and fusiform gyrus while viewing emotional faces.

Additionally, all the studies described here examined specific factors that might influence stress susceptibility in healthy individuals, such as gender and genotype. Stress is known to differentially influence women and men at the behavioral level (Lighthall et al., 2012; Matud, 2004), hormonal level (Kirschbaum, Wust, \& Hellhammer, 1992) as well as neural level (Wang et al., 2007). Sex hormones, such as estrogen and progesterone, are known to affect emotions and cognition, contributing to sex differences in behavior. Females are also known to respond differently to stress than males in relationship to the phase of the menstrual cycle (Kajantie \& Phillips, 2006). Animal studies also provide an extensive repertoire of information on gender differences in stress response, especially in terms of HPA axis activity. Animal studies have reported differences in stress hormone receptor densities within brain regions, especially the hippocampus, between genders (Kitraki, Kremmyda, Youlatos, Alexis, \& Kittas, 2004). Higher amounts of corticosteroid binding globulins have been reported in females compared to males as well. These corticosteroid binding globulins are known to be positively regulated by estrogen and stress (N C Vamvakopoulos \& Chrousos, 1993; Nicholas C Vamvakopoulos \& Chrousos, 1994). As a result, possible gender effects on the neural correlates of stress response during cognition were of interest in the current studies. Furthermore, research has indicated that the presence of the short allele variant (S-allele) on the promoter region of serotonin transporter gene (SLC6A4) polymorphism (5HTTLPR) confers increased stress susceptibility (Hariri \& Holmes, 2006; Karg, Burmeister, Shedden, \& Sen, 2011; Mueller 
et al., 2011). Research also shows increased methylation for this gene in new born females compared to males, pointing towards a possible underlying mechanism for gender related differential stress susceptibility (Dukal et al., 2015). Therefore, the studies described here explored the functional connectivity changes in brain regions during cognitive task performance following stress exposure while taking into consideration common stress susceptibility factors like gender and genotype. 


\section{CHAPTER TWO:}

\section{EFFECTS OF STRESS ON FUNCTIONAL CONNECTIVITY DURING VERBAL PROCESSING}

\section{INTRODUCTION}

The stress response is the body's normal physiological reaction to a situation that is perceived as threatening or overwhelming (Lucassen et al., 2014). While the stress response serves to promote survival in challenging situations, prolonged exposure is known to have a major impact on an individual's physiological and psychological wellbeing. For example, chronic stress has been linked to cardiovascular diseases, hypertension, anxiety and depression, among many other conditions (Cohen et al., 2007; Dimsdale, 2008; Epel et al., 2004; Kemeny, 2003; McEwen, 2007), and short term exposure to stress, or acute stress, is known to have negative effects on cognitive functioning, working memory and other executive functions (McEwen \& Saplosky, 1995; Sandi, 2013). Since the brain is the first organ that perceives stress and initiates the stress response, a greater understanding of how stress affects brain activity is important in order to understand the mechanisms by which certain individuals may be more susceptible to the deleterious effects of stress. This would, in turn, help in the development of more effective, individualized treatment approaches to address the issue of stress susceptibility.

The effects of stress are primarily modulated through two systems - the hypothalamic pituitary - adrenal (HPA) axis and the locus coeruleus-norepinephrine system. The 
norepinephrine system activates the sympathetic nervous system and releases

norepinephrine and epinephrine within seconds in response to a stressor. The HPA axis, on the other hand, responds to short-duration acute stressors by stimulating the release of cortisol, a response that takes minutes to occur (Kemeny, 2003). While the HPA axis response can be triggered even in anticipation of stressful events and is responsible for chronic buildup of negative responses as mentioned earlier, the rapid response to immediate stressors that the locus coeruleus-norepinephrine system produces is transient (Andrews, Ali, \& Pruessner, 2013). Previous research has also demonstrated a significant interaction between the locus coeruleus-norepinephrine system and the HPA axis during stress exposure, where administration of a beta-adrenergic antagonist, thereby suppressing locus-coeruleus-norepinephrine activity, also increased HPA activity (Andrews \& Pruessner, 2013). These mechanisms contribute to the detrimental effects of stress observed in cognitive tasks in healthy individuals with no known anxiety disorder.

Several studies have explored the effects of stress on neural activation in the brain. Pruessner et al. (2008) showed that an acute stressor had effects on resting state brain activation and was associated with extensive deactivation of the limbic system. This observation was found to be directly correlated with cortisol levels in the participants. Previous research has also examined effects of psychological stress on hippocampal activation during memory tasks (Khalili-Mahani, Dedovic, Engert, Pruessner, \& Pruessner, 2010; S. Qin, Hermans, van Marle, \& Fernandez, 2012), and reduction in reward-related prefrontal function during a monetary incentive delay task (Ossewaarde et al., 2011). Studies looking at functional connectivity changes within the brain associated 
with stress exposure have reported increased coupling of networks integrating autonomicneuroendocrine control and vigilant attentional reorienting in the presence of stressrelated noradrenergic activity (Hermans et al., 2011). Acute psychological stressors were also reported to enhance resting state connectivity of the amygdala with the dorsal anterior cingulate cortex, anterior insula and with the anatomical region corresponding to the locus coeruleus (H. J F van Marle et al., 2010). However, the effects of acute stress on neural activation and functional connectivity during cognitive tasks that require rapid access to multiple networks, such as language processing tasks accessing semantics and lexical networks, have not been explored.

The specific effects of stress on cognitive tasks that require searching through multiple networks are of interest because increased stress leads to a state of heightened arousal and consequently, narrowed, focused attention (Easterbrook, 1959; Koob, Cole, Swerdlow, Le Moal, \& Britton, 1990). As a result, the ability to access multiple, remote networks to come up with more creative solutions to difficult problems is hampered (Heilman, Nadeau, \& Beversdorf, 2003; Hillier, Alexander, \& Beversdorf, 2006; Kelley, Yeager, Pepper, \& Beversdorf, 2005; Narayanan et al., 2012; Renner \& Beversdorf, 2010). A primary example of this can be seen in the effects of stress on language tasks, such as public speaking and test taking. The noradrenergic system appears to be critical for this effect, as evidenced by the therapeutic effects of beta-adrenergic antagonists for individuals with stress-induced dysfunction with these tasks (Faigel, 1987, 1991; Laverdure \& Boulenger, 1991). In the current study, we specifically looked at verbal fluency tasks because successful completion of these tasks requires rapid searching 
through lexical and semantic networks to determine appropriate responses to specific cues (Weiss et al., 2004). This process would be directly affected by stress (J. K. Alexander et al., 2007), and may show corresponding effects on brain activity and function. For instance, manipulation of the locus coeruleus-norepinephrine system through administration of a beta-adrenergic antagonist has been shown to effectively reverse the negative effects of stress on cognitive functioning during verbal problem solving (J. K. Alexander et al., 2007; Faigel, 1991) as well as other tasks requiring greater cognitive flexibility (Beversdorf, White, Chever, Hughes, \& Bornstein, 2002; Hecht, Will, Schachtman, Welby, \& Beversdorf, 2014; Heilman et al., 2003; Kelley et al., 2007). Previous work from our lab indicates that these performance benefits may be associated with improved functional connectivity between brain regions during cognitive processing (Narayanan et al., 2010), suggesting altered information processing. The results of such a study, looking at neural correlates for the effect of stress on language networks (specifically tasks requiring extensive searches through multiple networks) in healthy individuals may, therefore, provide evidence for targeted interventions to counter the negative effects of stress during such tasks. This would then have specific implications for understanding and addressing conditions such as public speaking anxiety and test taking anxiety (Faigel, 1987, 1991; Laverdure \& Boulenger, 1991) along with broader implications towards understanding effects of stress on the brain.

Furthermore, factors that increase an individual's susceptibility to stress, such as genetic predisposition, may modulate the aforementioned changes in information processing in the brain. For example, polymorphisms on the serotonin transporter gene, SLC6A4, have 
been shown to contribute to atypical stress responses (Caspi, Hariri, Holmes, Uher, \& Moffitt, 2010). The SLC6A4 gene has been shown to be highly polymorphic. The most extensively studied polymorphism is a 43 base-pair insertion/deletion polymorphism located in the promoter region of the gene (5-HTTLPR). The 43 base pair deletion, commonly referred to as the short allele (S-allele), results in a reduction in transcriptional efficiency and thus decreases expression of the gene by roughly $50 \%$ when compared to the 43 base pair insertion, referred to as the long allele (L-allele) (Bengel et al., 1998; Kenna et al., 2012; Lesch et al., 1996). Research has shown that individuals with the Sallele may be at higher risk of developing depression and anxiety related disorders when exposed to stress compared to those with the L-allele (Drabant et al., 2012; Duman \& Canli, 2015; Franklin, Saab, \& Mansuy, 2012; Mcguffin, Alsabban, \& Uher, 2011), and exhibit altered functional connectivity patterns following exposure to stress (van der Meer et al., 2016). Though a later study reported no association between the S-allele, stress exposure, and elevated risk for depression (Risch et al., 2009), a subsequent larger meta-analysis provided further evidence in support of the role for S-allele in increased risk for depression (Karg, Burmeister, Shedden, \& Sen, 2011). Furthermore, a study by Hariri et al. (2002) showed that participants with the S-allele had 5 times greater activation in the amygdala in response to fearful faces compared to participants with the L-allele, even when there was no difference in baseline anxiety- or fear-related traits between the groups. 5-HTTLPR polymorphisms have also been shown to impact prefrontal control of the amygdala (Volman et al., 2013), suggesting altered network dynamics in the brain. In an exploratory pilot project, we found that subjects with the Sallele performed worse on verbal fluency tasks under stress (Beversdorf et al., 2018). 
Therefore, examining how stress and genetics interact to affect imaging correlates during an established language task is of interest, which may lead to individualized treatment strategies in the future.

Gender is another important factor that, along with genotype, may modulate effects of stress on the brain. Previous studies have reported relevant differences in neural activation in males and females during cognitive tasks. For example, greater neural activation in relevant brain regions was reported in males than in females during a working memory task (Bell, Willson, Wilman, Dave, \& Silverstone, 2006) and a covert phonological verbal fluency task (Gauthier, Duyme, Zanca, \& Capron, 2009). It is possible that these differences in neural activation during task performance may be interrelated with genetic predisposition. For instance, Dukal et al. (2015) showed that new born females have higher methylation of SLC6A4 compared to newborn males, indicating potentially reduced SLC6A4 activity. This effect is proposed to be one of the driving forces contributing to increased incidence of depression in females compared to males (Bebbington, 1998; Bromet et al., 2011; Kessler, 2003; Kessler, McGonagle, Swartz, Blazer, \& Nelson, 1993; Weissman \& Klerman, 1977). Correspondingly, gender differences have also been reported in the neural correlates of stress response. Kogler et al., (2017) found that under stress, males exhibited greater activation in regions associated with "emotion and stress regulation, self-referential processing and cognitive control" compared to females, suggesting gender-specific effects. Thus, stress effects on information processing in the brain, especially for tasks such as verbal fluency that require dynamic access to multiple brain networks, may be modulated by additional 
factors that mediate stress susceptibility, such as gender and genetic predisposition, which warrants further investigation.

In the current study, we examined how stress affects neural activation and functional connectivity (FC) in the brain using functional magnetic resonance imaging (fMRI) during verbal fluency tasks. We also explored whether these measures differed in males and females with and without genetic polymorphisms associated with an atypical stress response. Based on the results from our previous work (J. K. Alexander et al., 2007), we predicted that performance on the verbal fluency task would be significantly impaired under stress, and performance deficits would be associated with decreased neural activation and FC in areas critical for language processing such as the left and right inferior frontal gyrus, left middle temporal gyrus, left parietal lobe and the left fusiform gyrus. We also predicted that females and individuals with at least one copy of the short allele of the serotonin transporter gene would exhibit a larger stress associated decrease in performance and associated changes in brain activation and FC compared to males and individuals lacking the short allele, respectively.

\section{MATERIALS AND METHODS}

\section{Participants}

Forty-five participants (Mean age $=19.5 \pm 1.6$ years) were recruited through an on-line subject pool at the University of Missouri. Participants without any previous medical or psychiatric history (including no history of anxiety-related disorders) were included in 
the study. All participants signed informed consent to participate and protocols were approved by the University of Missouri Health Sciences Institutional Review Board. Participants were administered the North American Adult Reading Test (NAART) (Uttl, 2002) to estimate full-scale intelligence quotient (IQ).

\section{Genetic Screening and Genotyping}

At the first session, participants were asked to provide a cheek swab to perform genetic testing. As with our previous work, genomic DNA was extracted using standard methods (Flexigene kit; Qiagen) (Hecht et al., 2016). Participants with the s/s and s/l genotype were categorized into the $S$-allele group and participants with the $1 / 1$ genotype were in the L-allele group for analysis. Apart from genotyping for s/s, s/1 or 1/1, we also looked for the single-base substitution, rs25531, A/G, in the 1/1 genotype. This single base substitution is shown to behave similarly to the short allele (Kenna et al., 2012). As such, one participant with the $1 / 1$ genotype and the rs $25531 \mathrm{~A} / \mathrm{G}$ substitution was grouped with the s-allele participants. Additionally, we also checked for the T/G single base substitution on rs3813034 (Gyawali et al., 2010) and presence of STin2.10 in place of STin2.12 in Intron 2 VNTR in 1/1 genotype group since these variations are known to show less efficient activity, similar to the short allele group (Murphy \& Moya, 2011). However, none of the participants were regrouped based on these specific variants.

\section{MRI Acquisition and Verbal Fluency Assessment}

Following genotyping, the participants were invited to take part in the fMRI portion of the study. To allow for within-subject comparison, the participants attended two separate 
imaging sessions in a blinded, counterbalanced manner. One-half of the participants attended the no stress control session on day one and the stress inducing session (stress session) on day two. The other half had the stress inducing session on their first day and the no stress control session on the second day. For each of the participants, the imaging sessions were separated by at least 24 hours. Participants were asked to refrain from alcohol, caffeine and nicotine for at least 12 hours prior to the imaging session.

Participants were also unaware that the tasks in one of the sessions was meant to induce stress. As outlined below, they were simply told they could take as much time as they needed for the tasks on one day, without any feedback regarding their performance, and that on the other day they would be timed and provided performance feedback. All participants were debriefed about the stress induction at the end of the study. Participants completed the State-Trait Anxiety Inventory (STAI) as a measure of level of anxiety or stress they were experiencing pre and post each imaging session.

Images were collected on a 3T Siemens Trio Scanner at the University of Missouri Department of Psychological Sciences Brain Imaging Center. Structural T1-weighted images were acquired for anatomical localization (MPRAGE, TR=1920 ms, TE=2.9 ms, Angle $=9$ degrees, FOV/matrix size $=256 \times 256,176$ sagittal slices at $1 \mathrm{~mm}^{3}$ resolution) . Functional $\mathrm{T} 2 *$-weighted images were acquired to measure the blood oxygenation level dependent $(\mathrm{BOLD})$ response $\left(\mathrm{EPI}, \mathrm{TR}=2000 \mathrm{~ms}, \mathrm{TE}=30 \mathrm{~ms}\right.$, Flip angle $=90^{\circ}, \mathrm{FOV}=256 \mathrm{x}$ 256, matrix size $=64 \times 64,32 \mathrm{AC}-\mathrm{PC}$ aligned slices at $4 \mathrm{~mm}^{3}$ resolution). 
During the stress session, participants were exposed to an fMRI-compatible stressor, the Montreal Imaging Stress Test (MIST) (Dedovic et al., 2005) to induce stress while in the scanner. The MIST, based on the Trier Social Stress Test (TSST) (Kirschbaum, Pirke, \& Hellhammer, 1993), involves a number of timed mental arithmetic tasks with a social evaluative threat component (Dedovic et al., 2005). The difficulty of the mental arithmetic tasks was set for each individual based on their practice runs to give a success rate of approximately 50\%. Participants were given performance feedback in the form of a performance indicator on their screen along with negative feedback from the experimenter after each run. During the no stress control session, easier arithmetic problems were presented without any time limit and no feedback or social evaluative prompts were provided. The MIST stressor or control task were interspersed with 30second TASK blocks. During the TASK block, the participants were asked to perform categories fluency task in which they were asked to name as many words as they could think of that belong to a certain category (e.g.: animals, vegetables) (Strauss et al., 1998). The prompt appeared on the screen for the entire duration of each block. Subject responses were recorded offline and the total number of words generated during each block were noted. The task block was followed by a 30 second REST block where the participants were asked to fixate on cross hairs displayed on the screen and no other stimulus or task demands were provided. Following the 30 second REST block, the MIST-TASK-REST block pattern was repeated two more times for each run (Figure 2.1). Following the categories fluency task, the participants performed letter fluency task, where the task block involved naming as many things as they could think of that started with a target letter (e.g., F, A, S) (Strauss et al., 1998). Participants underwent two 
iterations each of the letters task and the categories task in each of the two sessions (Figure 2.1), and different target letters or categories were presented in each block. Participants were given practice sessions to familiarize them with the tasks prior to the start of the imaging session.

Figure 2.1 Stimulus protocol design for the letter and category fluency tasks, initially interleaving MIST and REST blocks with category fluency blocks (animals, things to wear, food), then interleaving MIST and REST blocks with letter fluency blocks (F, A, S), then repeating the sequence with a second set of category and letter fluency blocks.
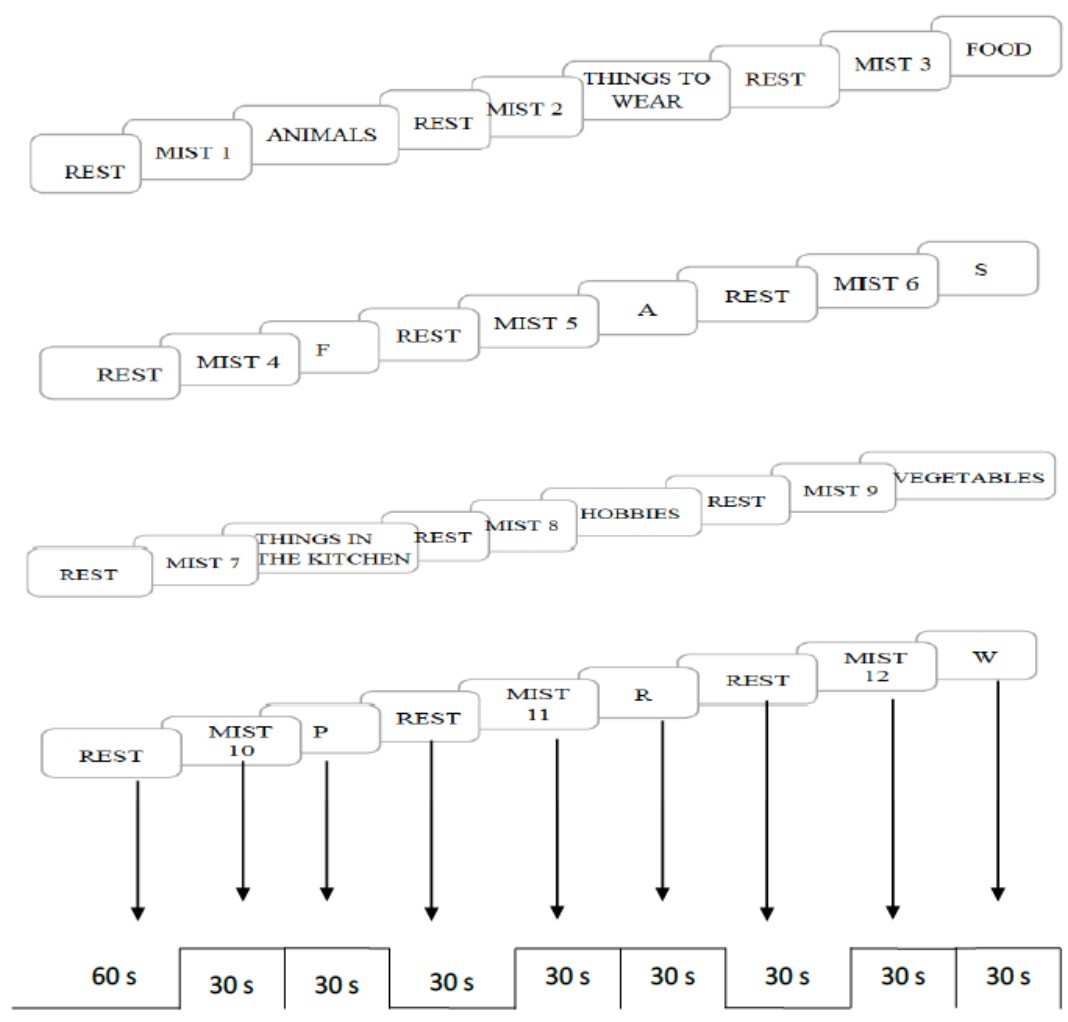

MRI Analysis

Functional magnetic resonance imaging data were analyzed using FEAT (FMRI Expert Analysis Tool) Version 6.00, part of FSL (FMRIB Software Library, 
www.fmrib.ox.ac.uk/fsl). Registration to each participant's high resolution structural image and standardized MNI space was carried out using FLIRT (FMRIB's Linear Image Registration Tool) (Jenkinson, Bannister, Brady, \& Smith, 2002; Jenkinson \& Smith, 2001). Preprocessing consisted of brain extraction using BET (Brain Extraction Tool) ( (Smith, 2002), slice timing correction (interleaved) using Fourier-space time-series phase-shifting, motion correction using MCFLIRT (Motion Correction using FLIRT) (Jenkinson et al., 2002), Gaussian spatial smoothing (FWHM of $5 \mathrm{~mm}$ ), high pass temporal filtering (Gaussian-weighted least-squares straight line fitting, cutoff $100 \mathrm{~s}$ ) and grand-mean intensity normalization of the entire 4D dataset. Participants who had relative motion greater than $2 \mathrm{~mm}$ along either of the $\mathrm{x}-, \mathrm{y}$ - or $\mathrm{z}$ - axis or the rotational axes (roll, pitch and yaw) were excluded from further analysis. Z (Gaussianised T) statistic images were thresholded using clusters determined by $\mathrm{Z}>2.3$ and a (corrected) cluster significance threshold of $\mathrm{P}=0.05$ (Worsley, 2001).

Masks centered on the a priori regions of interest (ROIs) (left and right inferior frontal gyri, the left middle temporal gyrus, the left parietal lobe and the left fusiform gyrus, see Figure 2.2) were created using the Harvard-Oxford Cortical Structural Atlas tool in FSL. First, to see if there were effects of stress on individual ROIs, which might interact with FC, we examined ROI activation across stress conditions. Percentage change in BOLD signal within the ROI during task blocks in relation to rest was extracted using the FEATQUERY tool of FSL for each individual subject. In order to analyze functional connectivity, the time series from the voxel of maximum activation within the ROI during task blocks was extracted using FEATQUERY for each individual subject, as 
described in Tivarus, Hillier, Schmalbrock, \& Beversdorf (2008). The analyses were conducted on time series extracted from within task blocks to account for the effect of activation magnitude differences between stress conditions on FC. Following extraction of time points within the task block in the analysis, we ran bivariate correlations between the time series for each of the ROI pairs, and the correlation coefficients were converted using Fisher's r-to-z-transformation to examine functional connectivity strength. For overall functional connectivity, average z-score across all ROI pairs was computed for each individual subject separately for the stress and no stress conditions.

Figure 2.2 Regions of interest created using the Harvard-Oxford Cortical Structural Atlas tool in FSL. Red: Left inferior frontal gyrus. Green: Left middle temporal gyrus. Purple:

Left parietal lobe Blue: Left fusiform gyrus

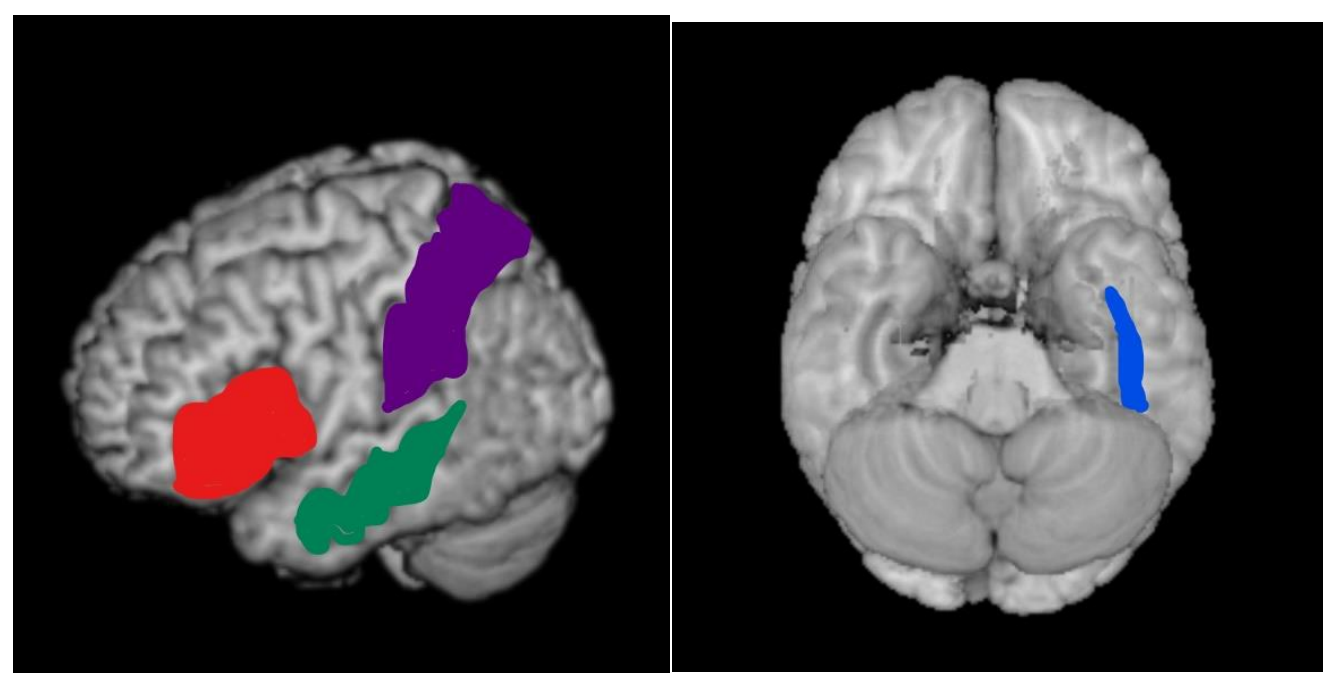

Statistical Analysis 
A series of repeated measures multivariate analysis of variance (MANOVA) tests (SPSS ver 24, IBM Corp.) was used to examine differences in (1) activation in the regions of interest (2(stress, no stress)*5 (ROIs)), (2) average functional connectivity across ROIs (2-way MANOVA, (stress, no stress)*overall functional connectivity) and (3) functional connectivity strength between individual ROI pairs (2 (stress, no stress)*10 (ROI pairs)) between the stress and no stress conditions. Both gender and allele type were included as between subject factors in all the aforementioned MANOVAs. Because previous research has reported on the effects of intelligence and age on neural correlates of cognitive processing and stress reactivity, respectively (Aldwin, C.; Sutton, K.; Chiara, G. \& Avron, 1996; Neubauer \& Fink, 2009; Rodríguez-Aranda \& Martinussen, 2006; Rudolph \& Hammen, 1999; Sapolsky, Krey, \& McEwen, 1986), these analyses were repeated, as MANCOVAs, including age and IQ as covariates. Correlations between performance and activation magnitude and functional connectivity strengths were also examined. All results were Bonferroni corrected for multiple comparisons.

\section{RESULTS}

\section{Demographics}

For the letters task, fMRI data from 36 participants were analyzed. 9 participants were dropped from the analysis due to either excessive motion in the scanner $(n=2)$ or because they did not attend the second session $(n=7)$. Among the participants whose data was analyzed for the letters tasks, there were 12 males (7 S-allele (S/S or S/L genotype), 5 Lallele (L/L genotype)) and 24 females (12 S-allele, 12 L-allele). There was no difference 
in age or IQ between the S-allele and L-allele groups or between males and females, $\mathrm{p}>$ 0.05 in all instances (Table 2.1).

For the categories task portion of this study, fMRI data from 37 participants were analyzed. 8 participants were dropped from the analysis due to excessive motion in the scanner $(n=3)$ or because they did not attend the second session $(n=5)$. Among the participants whose data was analyzed, there were 13 males and 24 females. Furthermore, 16 participants had the S-allele of the 5-HTTLPR (11 females and 5 males).

Table 2.1 Demographics of participants

\begin{tabular}{|c|c|c|c|c|c|c|c|c|c|}
\hline & $\begin{array}{c}\text { Total } \\
(\mathbf{n}=36)\end{array}$ & Male & Female & $t(d f)$ & $\mathrm{p}$ & $\begin{array}{c}\text { S-allele } \\
(\mathrm{n}=17)\end{array}$ & $\begin{array}{c}\text { L-allele } \\
(\mathrm{n}=19)\end{array}$ & $t(d f)$ & $\mathrm{P}$ \\
\hline $\begin{array}{c}\text { AGE } \\
\text { (YEARS) }\end{array}$ & $\begin{array}{l}19.7 \\
(1.7)\end{array}$ & $\begin{array}{l}19.5 \\
(1.9)\end{array}$ & $\begin{array}{l}19.8 \\
(1.5)\end{array}$ & $-0.278(34)$ & 0.783 & $\begin{array}{l}19.7 \\
(1.2)\end{array}$ & $\begin{array}{l}19.7 \\
(1.9)\end{array}$ & $-0.438(34)$ & 0.664 \\
\hline $\begin{array}{c}\text { GENDER } \\
\text { (M/F) }\end{array}$ & $12 / 24$ & - & - & - & - & $5 / 12$ & $7 / 12$ & $0.223^{* *}$ & 0.637 \\
\hline $\begin{array}{c}\text { IQ } \\
\text { (NAART) }\end{array}$ & $\begin{array}{l}107.6 \\
(4.9)\end{array}$ & $\begin{array}{l}108.9 \\
(4.9)\end{array}$ & $\begin{array}{l}106.9 \\
(4.7)\end{array}$ & $1.432(34)$ & 0.161 & $\begin{array}{l}109.2 \\
(5.3)\end{array}$ & $\begin{array}{l}106.4 \\
(4.3)\end{array}$ & $1.273(34)$ & 0.212 \\
\hline \multicolumn{10}{|c|}{ ** CHI-SQUARE STATISTIC } \\
\hline \multicolumn{10}{|c|}{$\begin{array}{l}\text { GROUP COMPARISONS WERE PERFORMED USING INDEPENDENT SAMPLES T-TEST. ALL VALUES } \\
\text { ARE SHOWN AS MEAN(SD) UNLESS OTHERWISE INDICATED. }\end{array}$} \\
\hline
\end{tabular}

Paired sample t-tests did not show any significant differences on the State $(t=0.411$, $\mathrm{p}=0.684)$, Trait $(\mathrm{t}=-1.577, \mathrm{p}=0.124)$ or difference between State and Trait $(\mathrm{t}=1.257$, $\mathrm{p}=0.217$ ) anxiety scores pre and post imaging session. Within the no stress condition, trait anxiety was found to be significantly greater compared to state anxiety $(\mathrm{t}=-2.611$, $p=0.013)$. 


\section{Number of words generated}

Contrary to our expectations, there was no difference between the number of words generated under stress and no stress by the participants performing the letters task (stress: $\operatorname{Mean}(\mathrm{SD})=45.69(14.3)$, no stress: $\operatorname{Mean}(\mathrm{SD})=44.50(10.9) ; t=-0.663, p=0.512)($ responses were not available for 4 participants) or categories task (stress: Mean(SD)=57.39(12.3), no stress: $\operatorname{Mean}(\mathrm{SD})=56.97(12.9) ; t=-0.157, p=0.876)$ (responses were not available for 5 participants).

\section{ROI Activation}

Group activation maps while performing the letters fluency tasks under (a) stress and (b) no stress are shown in Figure 2.3.

Figure 2.3 Group activation maps obtained from participants while performing the letters fluency task under (a) stress and (b) no stress

(a)
Thresholded activation images
2.3
5.9

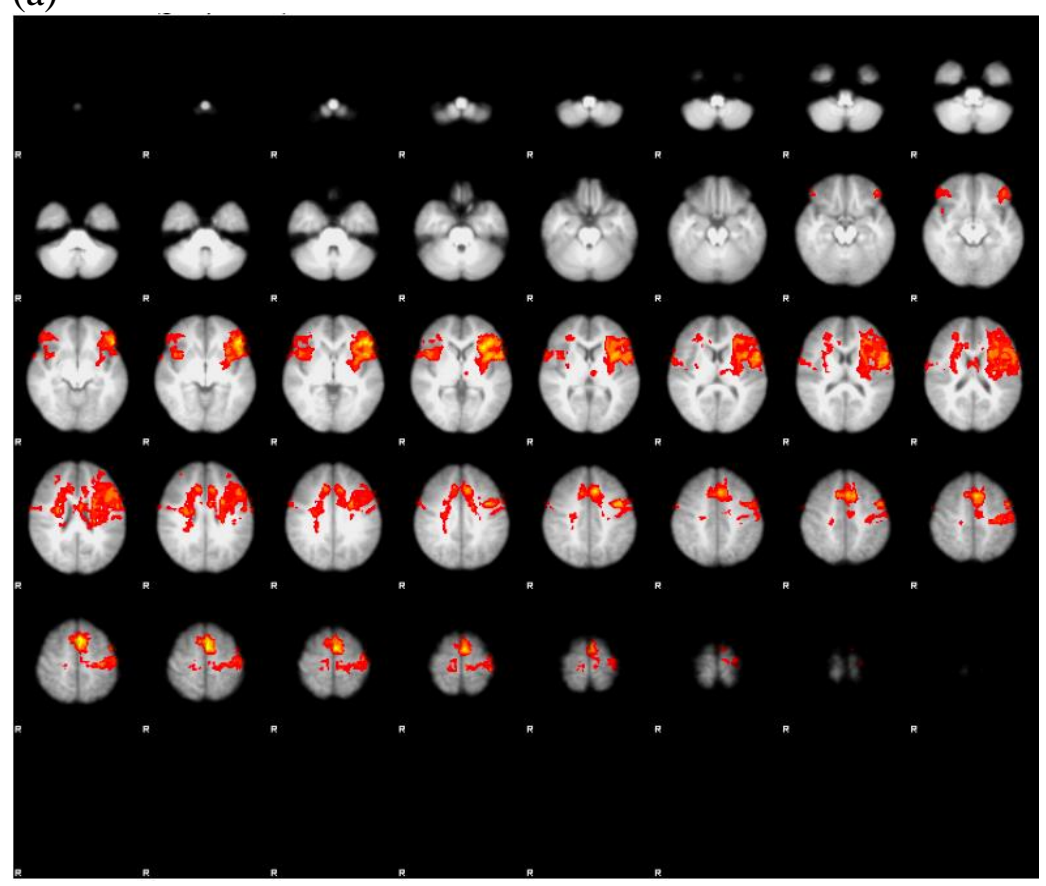




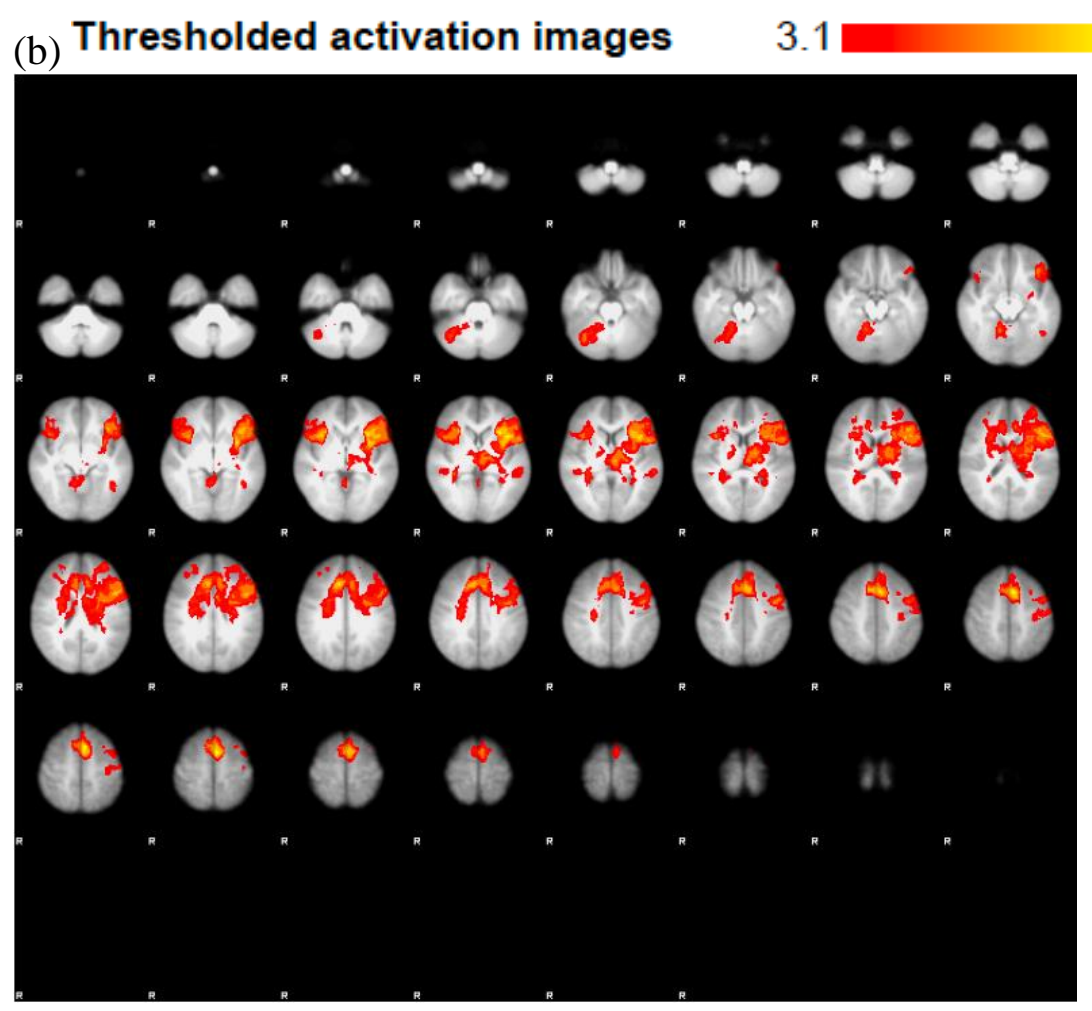

For the letters task, a repeated measures MANCOVA did not reveal any main effect of stress across all ROIs $(F(9,21)=1.506, p=0.210)$, nor were there any overall interactions with gender $(F(9,21)=1.475, p=0.221)$ or genotype $(F(9,21)=0.381, p=0.381)$. However, to determine whether there were effects on individual ROIs, we examined each ROI separately, corrected for multiple comparisons. Stress was found to increase percentage signal change in the left inferior frontal gyrus $(F(1,29)=5.157, p=0.031)$ and decrease percentage signal change in the left parietal lobe $(F(1,29)=8.389, p=0.007)$, when age and IQ were included as covariates in the analysis. Males showed greater activation performing the task under stress compared to females $(F(1,29)=4.581, p=0.024)$ in the left fusiform gyrus. A similar effect was observed in the left inferior frontal gyrus when age and IQ were included as covariates in the analysis $(F(1,29)=6.168, p=0.019)$. In the 
left middle temporal gyrus, males with the S-allele had greater activation under stress compared to females with the $S$-allele $(F(1,29)=5.707, p=0.024)$.

For the categories task, a repeated measures MANCOVA did not reveal any main effect of stress across all ROIs $(F(9,21)=2.097, p=0.078)$, nor were there any overall interactions with gender $(F(9,21)=1.084, p=0.414)$ or genotype $(F(9,21)=0.807$, $p=0.615)$. The effect of stress on individual ROIs was then examined, corrected for multiple comparisons. There was a significant decrease in activation in the left inferior frontal gyrus when performing the task under stress compared to no stress control session $(F(1,28)=6.607, p=0.016)$. When age and IQ were included as covariates in the analysis, males showed increased activation in this region compared to females while performing the task under stress $(F(1,28)=4.510, p=0.043)$. We did not observe any effects in the other regions that were assessed.

\section{Functional Connectivity}

For the letters task, a repeated measures MANOVA revealed a trend towards an increase in overall $\mathrm{FC}$ strength across our a priori regions of interest $(F(1,31)=4.071, p=0.05)$, when performing letters task under stress compared to no stress (Figure 2.4a), but no significant interactions with gender $(F(1,31)=1.977, p=0.17)$ and allele type $(F(1,31)=0.959, p=0.33)$ were observed. To determine whether region specific effects were driving this overall trend, we next examined functional connectivity strength between each of the ROI pairs while performing the tasks under stress and no stress in a 
manner adjusted for multiple measures. Stress was found to increase the strength of functional connectivity between the left inferior frontal gyrus and the left fusiform gyrus while performing the task under stress compared to no stress session $(F(1,29)=7.499$, $p=0.01$ ) (Figure 2.4b). When age and IQ were included as covariates in the analysis, males were found to have greater strength of connectivity between the left parietal lobe and left fusiform gyrus $(F(1,29)=4.550, p=0.042)$ and the left inferior frontal gyrus and left fusiform gyrus $(F(1,29)=6.834, p=0.014)$ while performing the task under stress compared to females. Post hoc t-tests revealed that the functional connectivity strength increases between the left parietal lobe and left fusiform gyrus observed in males during task under stress was likely driven by age and IQ at the multivariate level.

Figure 2.4 Functional connectivity strength across ROIs during letters fluency task under stress vs. no stress (a) Overall FC strength across all ROIs showed a trend towards significance with increased strength of connectivity under stress compared to no stress. (b) Increased strength of functional connectivity was observed between the left inferior frontal gyrus and the left fusiform gyrus under stress. 


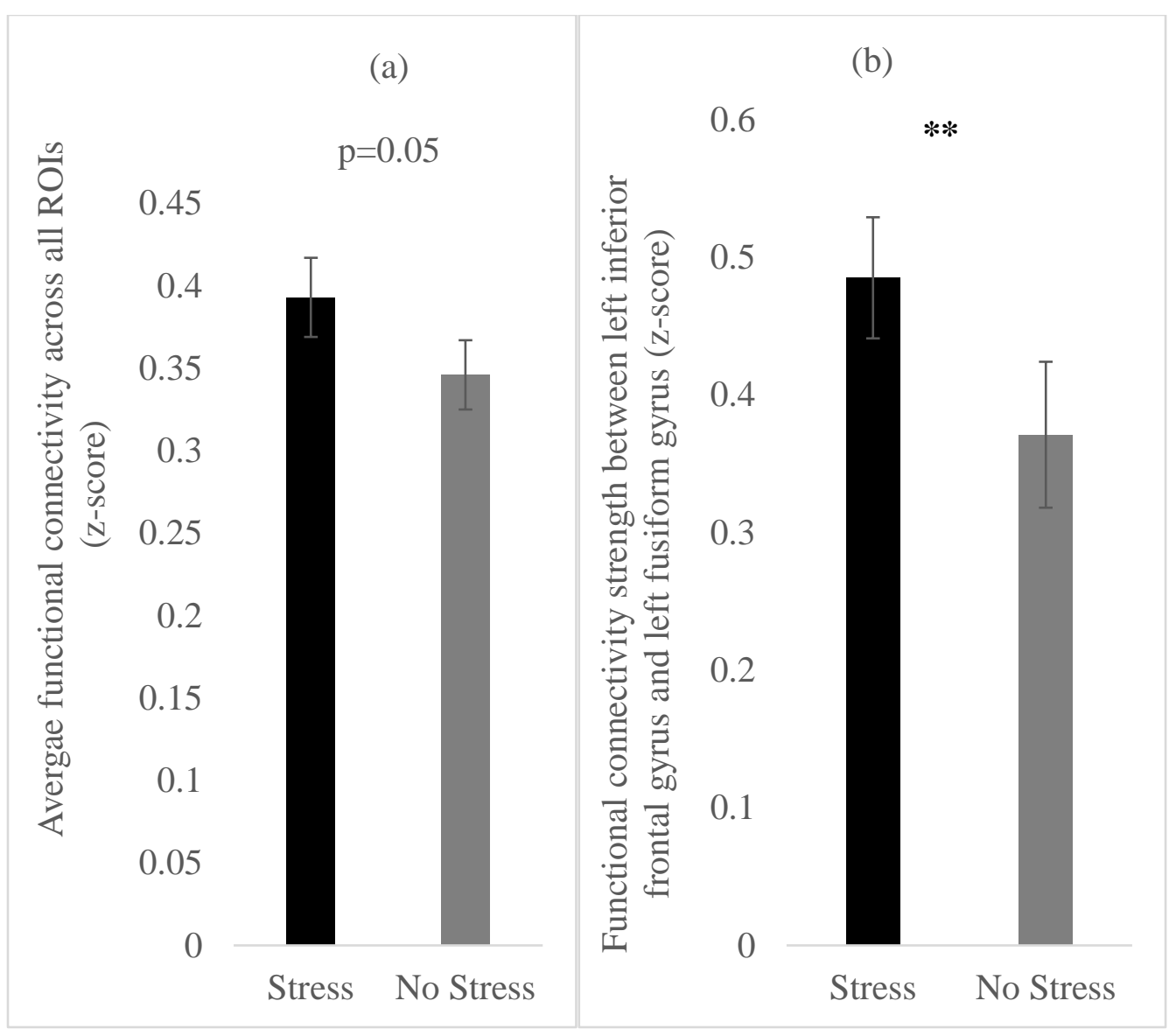

For the categories task, a repeated measures MANOVA showed no difference in overall functional connectivity strength across all ROIs under stress and no stress. Despite the lack of differences in overall functional connectivity strength across all ROIs under stress and no stress, we wanted to explore possible differences that might be present in individual ROI pairs under the two conditions with appropriate corrections for multiple comparisons. While no main effect for stress was observed on functional connectivity strength between our regions of interest, a MANCOVA revealed that participants with the S-allele had lower strength of functional connectivity between the inferior frontal gyrus and the left middle temporal gyrus under stress compared to participants with the 
L-allele performing the task under stress $(F(1,27)=4.238, p=0.049)$ (Figure 2.5). Only Lallele subjects produced a significant increase in functional connectivity between the two regions during task under stress compared to no stress in post-hoc t tests. Additionally, females with S-allele were found to have higher strength of functional connectivity between these regions when performing the task under stress compared to males with the S-allele performing the task under stress $(F(1,27)=5.593, p=0.025)$ at the multivariate level. Post-hoc t tests revealed that only L-allele females had a significant increase in strength of functional connectivity between the left inferior frontal gyrus and left middle temporal gyrus in response to performing the task under stress vs. no stress.

Figure 2.5 Functional connectivity strength across ROIs during category fluency task under stress vs. no stress. S-allele participants had lower strength of functional connectivity between the left inferior frontal gyrus and left middle temporal gyrus under stress compared to L-allele participants. 


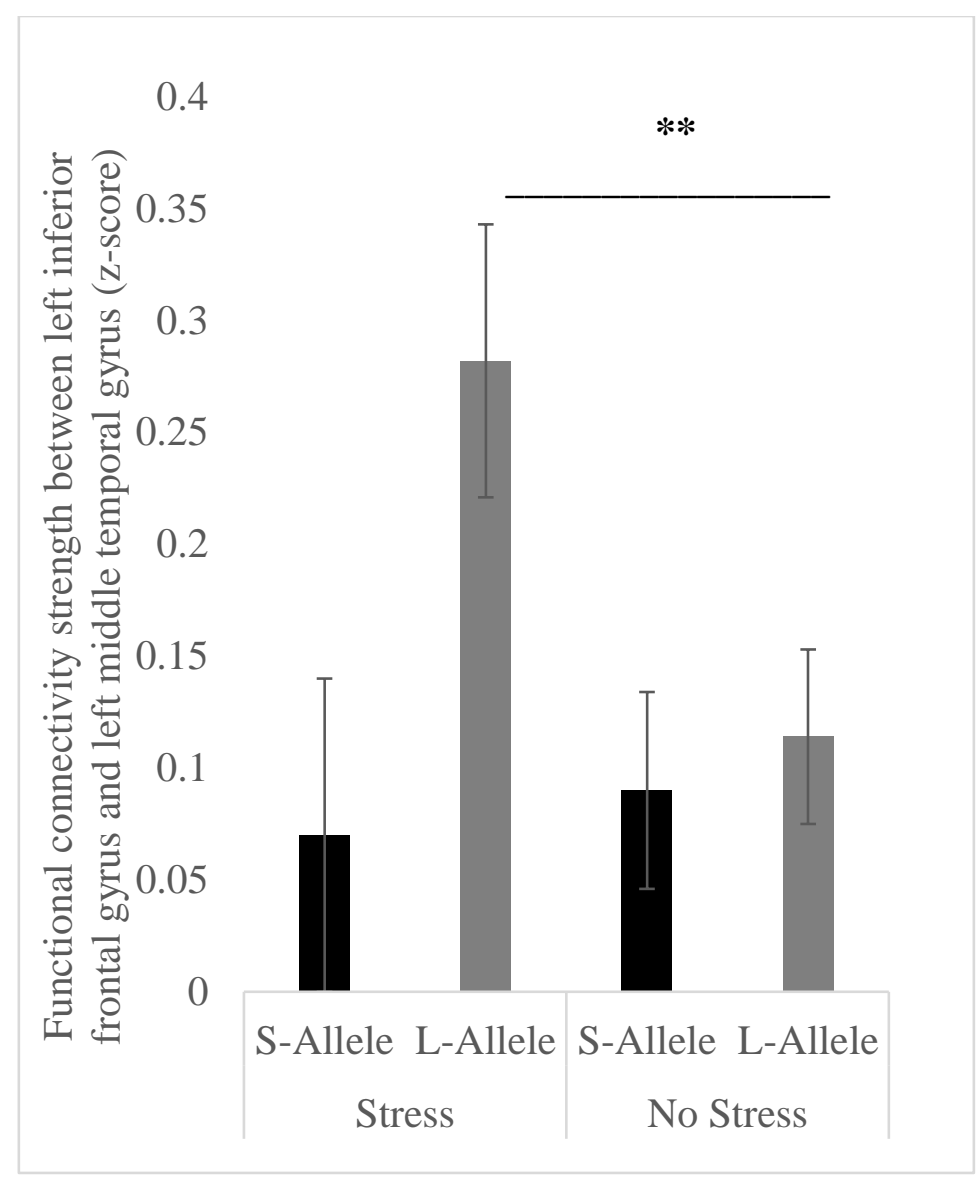

\section{Activation, functional connectivity and task performance}

While there were no effects of stress on performance on verbal fluency tasks, we wanted to examine the possibility of differences in the underlying neural correlates during stress. Therefore, in order to examine how changes in neural activation and functional connectivity under stress related to changes in performance on the tasks, we determined the partial correlations between difference in task scores and differences in functional connectivity strengths under stress and no stress. The difference in scores on the letters task under stress and no stress was found to be significantly correlated with changes in overall functional connectivity across ROIs under stress and no stress ( $\mathrm{r}=0.367, p=0.039)$. 
Difference in scores on the letters task under stress and no stress was not significantly correlated with differences in FC strength between individual ROI pairs under stress and no stress. No significant correlations were observed for difference in scores and difference in overall functional connectivity across all ROIs under stress and no stress for the categories task. However, difference in scores under stress and no stress for the categories task was significantly correlated with changes in FC between the left inferior frontal gyrus and left middle temporal gyrus $(\mathrm{r}=0.374, \mathrm{p}=0.038)$ and between the left middle temporal gyrus and the left fusiform gyrus $(\mathrm{r}=0.366, \mathrm{p}=0.043)$.

\section{DISCUSSION}

Stress, both chronic and acute, is associated with deleterious effects on an individual's physiological and psychological health. While prolonged stress exposure can lead to a myriad of problems such as anxiety and cardiovascular diseases (Cohen et al., 2007; Dimsdale, 2008; Epel et al., 2004; Kemeny, 2003; McEwen, 2007), short term exposure to stress is known to affect cognitive functioning, working memory and other executive functioning (McEwen \& Saplosky, 1995; Sandi, 2013). Stress is also known to affect the ability to flexibly engage brain networks in order to processing information that requires access to multiple remote resources (Alexander, Hillier, Smith, Tivarus, \& Beversdorf, 2007). It has been proposed that under stressful conditions, more dominant resources and pathways are utilized in order to narrow and focus attention (Easterbrook, 1959; Koob et al., 1990), thereby making it difficult to access more remote resources for creative problem solving. While studies have consistently demonstrated effects of acute stress on 
resting state functional connectivity within the brain (Fan et al., 2015; Liston et al., 2009;

J. C. Pruessner et al., 2008), there are no studies, to our knowledge, exploring how stress affects the brain during verbal processing tasks in healthy individuals. The effect of stress on verbal processing is of relevance for conditions such as public speaking and test taking anxiety (Faigel, 1987; Laverdure \& Boulenger, 1991) and has broader implications towards furthering the understanding and addressing of stress effects on the brain. This study aimed to explore the effects of stress on neural activation and functional connectivity in the brain during cognitive processing associated with a verbal processing in healthy individuals. The influences of genotype and gender on this effect were also explored.

The verbal fluency tasks used in this study were the letters task, where participants were asked to produce as many words as they could think of that started with a certain letter, and the categories task, where participants had to produce as many words as they could think of that belonged to a certain category. Successful completion of these tasks required searching through appropriate brain networks to determine what words do and do not meet the specified requirements and involves significant use of the frontal lobes (Weiss et al., 2004). Searching through networks of this type has been shown to be impaired by stress (Alexander, Hillier, Smith, Tivarus, \& Beversdorf, 2007). Our hypothesis was that due to the increased resting state FC with stress (Hermans et al., 2011), the FC induced during this task would be blunted during stress. Therefore, we used verbal fluency tasks to explore the effects of stress on functional connectivity during language processing. 
The current study revealed that acute stress influences both neural activation and functional connectivity during semantic and phonological processing associated with verbal fluency tasks, but the effects were not observed globally across all regions. There was an increase in functional connectivity strength between the left inferior frontal gyrus and the left fusiform gyrus during the letters task under stress. The left inferior frontal gyrus is known to be involved in phonological tasks, processing the sounds of words and letters (Fiez, 1997) and within the left inferior frontal gyrus, the expressive language area of the brain, Broca's area, plays a dynamic role in both language production and comprehension (Flinker, Korzeniewska, Shestyuk, Franaszczuk, \& Dronkers, 2015; Hagoort, 2014). As a result, previous studies have reported increased activation in this region during the letters tasks (Costafreda et al., 2006; Hirshorn \& Thompson-schill, 2006). Furthermore, the results from this study indicate that stress results in a greater increase in activation in the region. The left fusiform gyrus, on the other hand, is thought to be involved in recognizing words and letters from shapes and forms (Devlin, Jamison, Gonnerman, \& Matthews, 2006). An increase in functional connectivity strength between these two regions when performing the letters tasks under stress could therefore, be reflective of how stress places additional load on long distance functional connections in the brain during cognitive tasks. Further support for this argument comes from resting state studies looking at language networks indicating a predominance of short-range functional connectivity, suggesting that typically short distance connections are favored for routine language processing (Tomasi \& Volkow, 2012). Additionally, the increase in functional connectivity strength between the left inferior frontal gyrus and the left fusiform gyrus was found to be greater for males than females. Interestingly, males also 
showed an increase in strength of functional connectivity between the left parietal lobe and left fusiform gyrus under stress compared to females, driven by age and IQ, pointing towards a possible gender specific effect involving short distance connections. An overall decrease in activation, irrespective of gender, was observed in the parietal lobe, during letters task under stress. This could be reflective of reduced attentional processing in the region following the stress paradigm as has been observed previously (Hayashi, MizunoMatsumoto, Okamoto, Kato, \& Murata, 2012). The increased functional connectivity between the left parietal lobe and left fusiform gyrus in males can then be viewed as a possible compensatory mechanism to overcome this reduction in attentional processing via greater domain specific processing of semantic association information. Putting together this information with previous research reporting greater stress susceptibility for females (Klein \& Corwin, 2002; Matud, 2004), the results of the current study may begin to reveal specific neural foundations for greater stress susceptibility during cognitive tasks for females.

We noted a decrease in percentage signal change in the left parietal lobe during task under the no stress condition, with a greater decrease in activation corresponding to task performance under stress, irrespective of gender. The region of the parietal cortex that we focused on in this study comprised of Brodmann areas 7 (BA7) \& 40 (BA40) (Figure 2). These regions, apart from their role in attentional processing, are also known to be involved in phonologically encoded working memory. For this particular task it is likely that the participants employed a more semantically encoded form of working memory 
retrieval as opposed to phonologically encoded resulting in a relative "deactivation" in the area, as has been observed previously (Jonides et al., 1998).

For the categories task, regionally specific complex interactions were revealed for stress, genetics, and gender on functional connectivity strength. S-allele participants were shown to have decreased strength of functional connectivity between the left inferior frontal gyrus and the left middle temporal gyrus in response to performing the task under stress. The left inferior frontal gyrus, as mentioned previously, is involved in processing and generation of the sounds of words and letters (Fiez, 1997; Flinker, Korzeniewska, Shestyuk, Franaszczuk, \& Dronkers, 2015; Hagoort, 2014) and the left middle temporal gyrus is involved in multimodal integration of information (Bartha et al., 2003). Therefore, a decrease in connectivity strength in this short distance connection under stress for S-allele participants could indicate that stress affects the neural processes involved in interpretation and integration of information during the categories tasks to a greater degree in these participants. Post hoc t-tests revealed that only L-allele females produced a significant increase in functional connectivity strength between the left inferior frontal gyrus and the left middle temporal gyrus while performing the categories task under stress. However, again, this must be interpreted with caution given the small samples for the three-way interactions.

Although there were no differences observed in task performance measures for either the letters or the categories tasks under stress and no stress conditions, the differences in neural activation and functional connectivity indicate that there are specific neural 
mechanisms involved in verbal processing that are affected by stress. Additionally, the significant correlations between scores on the tasks and overall (letters task) and specific regional (categories task) functional connectivity strengths further support the effect of stress on neural correlates of verbal processing. Significant differences in scores on the STAI pre and post imaging session were not observed possibly due to the small sample size.

While previous research has shown effects of stress on resting state brain activation and connectivity (Hermans et al., 2011), effects of stress on other cognitive domains (KhaliliMahani, Dedovic, Engert, Pruessner, \& Pruessner, 2010; S. Qin, Hermans, van Marle, \& Fernandez, 2012; Ossewaarde et al., 2011), our findings here demonstrate the effects of stress and stress susceptibility factors, such as gender and allele-type, on brain activation and functional connectivity during established language tasks. This begins to reveal the basis for differences in stress susceptibility and tolerance in individuals and its impact on cognitive functioning. In general, long distance functional connections appeared to be strengthened to compensate for the additional load of the stressor, and males were found to exhibit stronger short distance functional connectivity between specific regions with stress. Understanding the neural basis of stress on cognitive functioning can facilitate our ability to help address the associated negative effects, notably in test and public speaking anxiety that require access to lexical and semantic networks under stressful conditions (Faigel, 1987; Laverdure \& Boulenger, 1991). Previous research from our lab has shown that norepinephrine antagonists such as propranolol, can help improve cognitive performance under stress (J. K. Alexander et al., 2007) and in tasks requiring high level 
of cognitive flexibility (Campbell et al., 2008). However, the response to these treatments still has a considerable amount of variability (Chamberlain \& Robbins, 2013). Based on the results of the current study, it would be important to see if stress susceptibility factors, such as gender and allele type, and the underlying neural correlates of increased stress susceptibility might influence the effectiveness of a pharmacological or nonpharmacological approach, a question also raised by the influence of genotype on the effects of stress on cognitive task performance (Beversdorf et al., 2018). This could then pave way for the development of individualized pharmacogenomics profiles that may help personalize treatments for individuals with increased susceptibility to stress. However, to better understand the implications of these findings, and the salience of the regional specificity, larger studies with tasks more sensitive to effects of stress will be needed to understand the relationship to performance. Additionally, other genetic markers will be important in subsequent studies, such as the COMT Val ${ }^{158}$ Met genotype, shown to alter the stress response in response to psychosocial stressors via dopamine processing (Hernaus et al., 2013), and also affect the impact of stress on working memory-related activity (Shaozheng Qin et al., 2012). Another factor to be considered in future studies would be the influence of early life stress (ELS) enhancing the effects of acute stress. Studies have shown an influence of ELS on resting state functional connectivity in the brain (Fan et al., 2014, 2015). The influence of ELS on functional connectivity was also found to have implications for therapeutic interventions such as determining the efficacy of using oxytocin to relieve stress in participants with and without ELS (Grimm et al., 2014). Furthermore, studies looking at neural underpinnings for maladaptive responses to stress exposure have noted volumetric and functional 
connectivity changes in the brain with implications for targeted therapeutic approaches (Admon et al., 2013).

In conclusion, this study is the first to explore stress effects on verbal processing with imaging markers and the influence of gender and genotype on this effect, which is of interest due to effects of stress on other types of language tasks such public speaking anxiety and test taking anxiety. Based on the results from studies looking at resting state and memory and reward-based tasks, we hypothesized that stress would be related to a decrease in functional activation in and connectivity between language regions of the brain during verbal fluency tasks. We also hypothesized that the stress related deficits would be greater in females and in participants with the S-allele. However, our results reveal more complex effects of stress, gender and genotype on functional connectivity between and neural activation within language regions of the brain while performing verbal fluency tasks. Long distance functional connections appeared to be strengthened to compensate for the additional load of the stressor, and males were found to exhibit stronger short distance functional connectivity between specific regions with stress. Overall, S-allele individuals and females were found to have greater stress associated decreases in mean amplitude of neural activation and strength of functional connectivity which is consistent with literature indicating that females and S-allele individuals are likely more vulnerable to the effects of stress, but the interpretation of this aspect is limited by the sample size for the 3-way interaction. Future studies will need to explore these complex interactions in detail in larger samples to draw more definitive conclusions. Also of interest would be to see how these results differ in individuals with 
known anxiety disorders. Understanding the neural underpinnings of effects of stress on cognitive processes, such as language processing discussed in this paper, combined with the potential for developing more effective therapeutic interventions can, in the future, significantly impact our understanding and treatment of disorders of increased stress susceptibility, such as anxiety disorders.

\section{LIMITATIONS}

The results of the study should be interpreted with caution, considering the following limitations of the study. The sample size was modest within some of the subgroups. Despite a moderate sample size, we were still able to observe significant effects of stress, gender and allele on functional connectivity during task performance. However, the small sample sizes in each group particularly limit interpretation of the three-way (stress $\mathrm{x}$ gene $\mathrm{x}$ gender) interactions. The MIST is known to induce stress in participants, as observed by previous reports of increased cortisol levels following MIST administration (Dedovic, Aguiar, \& Pruessner, 2009; J. C. Pruessner et al., 2008); however, reliable cortisol levels were not available in the current study, so obtaining this information will be important for future studies. Additionally, we focused on a priori regions of interest known to be involved in language processing in this preliminary study, but stress may also affect processing in other regions and networks, which warrants further investigation. It also needs to be noted that we did not observe significant differences in task performance for either of the fluency tasks under stress as compared to no stress. However, despite the lack of performance effects, the presence of specific neural differences in verbal processing associated with the 
presence of stress contribute towards our understanding of neural mechanisms of stress susceptibility.

\section{SUMMARY}

The current study explored functional connectivity and neural activation in language regions of the brain while performing verbal fluency tasks under stress. The influence of gender and genotype were also examined. The results of this study indicate that stress affects both neural activation and functional connectivity during language tasks. The study revealed that long distance functional connections in the brain may be strengthened to compensate for the additional load of the stressor. Males were also found to exhibit stronger short distance functional connectivity between specific regions with stress, in a possible gender specific compensatory effect. The study begins to demonstrate specific neural underpinnings for gender and genotype specific stress susceptibility. Larger studies looking at this information in more detail will hopefully pave the way for better methods to understand and address the mechanism and negative effects of stress susceptibility. 


\section{CHAPTER THREE:}

\section{EXPLORING FUNCTIONAL CONNECTIVITY CORRELATES OF VERBAL PROBLEM SOLVING UNDER STRESS}

\section{INTRODUCTION}

Decades of research have gone into understanding creativity, what mechanisms underlie the process of creative thinking, what makes certain individuals more creative than others and if there might be a potential link between creativity and psychopathology. In its simplest terms, creativity refers to the ability to flexibly access remote, scattered resources to come up with an innovative solution to a problem (Abraham, 2016). This process requires suppression of what might be the immediate, dominant response and searching for associations that might not be as obvious. Behavioral aspects such as REM sleep (Cai, Mednick, Harrison, Kanady, \& Mednick, 2009), posture (Lipnicki \& Byrne, 2005) and rate of blinking (Chermahini \& Hommel, 2010) are known to influence performance on tasks related to creativity in humans wherein the decreased noradrenergic tone associated with more relaxed states proves to be distinctly beneficial for creativityrelated tasks (Beversdorf, 2018). Pharmacological manipulations targeting betaadrenergic receptors in the noradrenergic system specifically modulated performance on convergent tasks involving the ability to search through widely distributed networks to come up with solutions on 'unconstrained' cognitive flexibility tasks (J. K. Alexander et al., 2007; Beversdorf, Hughes, Steinberg, Lewis, \& Heilman, 1999; Beversdorf et al., 2002). 
Stress is associated with increased noradrenergic tone, hypervigilance and narrowed, focused attention, because of which, creative thinking is impaired under stressful conditions (Martindale \& Greenough, 1973). Numerous studies have reported on the negative behavioral effects of stress on tasks requiring flexible thinking (McEwen \& Saplosky, 1995; Sandi, 2013; Shields, Sazma, \& Yonelinas, 2016). Correspondingly, manipulation of the noradrenergic system, using for example, the beta-adrenergic antagonist, propranolol, has been shown to rescue the negative effects of stress on cognition (J. K. Alexander et al., 2007; Faigel, 1991; Laverdure \& Boulenger, 1991). Propranolol has shown beneficial effects in creativity tasks that were difficult (Campbell et al., 2008), in easy tasks under conditions of likely upregulated noradrenergic activity such as cocaine withdrawal (Kelley et al., 2007), autism spectrum disorder where flexible access to networks maybe anatomically restricted (Beversdorf, Carpenter, Miller, Cios, \& Hillier, 2008) and Broca's aphasia (Beversdorf et al., 2007). The positive effects of such pharmacological interventions have been observed even in healthy individuals without any history of stress induced cognitive impairment or anxiety, indicating that these are fundamental processes of cognition not limited to patient populations.

Imaging techniques as well as focal cortical stimulation techniques have helped to better understand the neural basis of creative thinking as well as potential factors that influence an individual's ability to engage in creative thinking (Beaty et al., 2014; Gold, Faust, \& Ben-Artzi, 2012; Green et al., 2017; Green, Kraemer, Fugelsang, Gray, \& Dunbar, 2012; Jung-Beeman et al., 2004; Jung, 2013; Jung, Grazioplene, Caprihan, Chavez, \& Haier, 
2010; Kenett et al., 2018; Kühn et al., 2014; Zhao, Zhou, Xu, Fan, \& Han, 2014). A meta-analytic study by Mihov, Denzler, \& Förster, (2010), put forth evidence indicating the right hemisphere of the brain is more dominant in tasks of creativity. The results suggested that while the left hemisphere of the brain was involved in interpreting and understanding the information presented, the right hemisphere is more likely involved in associative processes necessary to come up with creative solutions. High levels of creativity have also been shown to be associated with increased functional connectivity between frontal regions engaged in cognitive control and the default mode network that is typically active during wakeful rest (Beaty et al., 2014). Further, Limb \& Braun, 2008 showed that spontaneous improvisation during a jazz piano performance was accompanied by decrease in activity in frontal regions involved in conscious control. Knowing the neural correlates of creative thinking then facilitates development of techniques to promote creative thinking in situations that may not be favorable for creativity, such as under situations of stress.

Numerous neuroimaging studies have explored the effects of acute stress on the brain both in its resting state as well as during cognitive task performance. For example, acute stress is associated with extensive deactivation of the limbic system during resting state (J. C. Pruessner et al., 2008). Hippocampal activation during memory tasks (Qin, Hermans, van Marle, \& Fernandez, 2012; Henckens, Hermans, Pu, Joels, \& Fernandez, 2009; M. Pruessner, Pruessner, Hellhammer, Bruce Pike, \& Lupien, 2007) and prefrontal activity in a delayed incentive task (Ossewaarde et al., 2011) were also found to be influenced by the presence of an acute stressor. Apart from activation in specific brain 
regions, the temporal correlations of activations between regions, also known as functional connectivity, is also affected by acute psychological stress. Increased coupling of networks integrating autonomic-neuroendocrine control and vigilant attentional reorienting (Hermans et al., 2011) and increased resting state connectivity of the amygdala with dorsal anterior cingulate cortex, anterior insula and locus coeruleus $(\mathrm{H}$. J F van Marle et al., 2010) have been reported under acute stress. However, how stress affects the functional connectivity in creative thinking has not been explored.

Some of the variability associated with creative abilities has been attributed to genetic components. For example, in a large study that looked at insight based creative problem solving, they found that single nucleotide polymorphisms (SNPs), specifically the A allele of rs4680 and $\mathrm{T}$ allele of rs4633, in the catechol-O-methyltransferase (COMT) gene was linked to greater scores on the task (Jiang, Shang, \& Su, 2015). The COMT enzyme encoded by this gene is involved in breaking down catecholamines such as dopamine and norepinephrine and was therefore, was of specific interest. The study also found that the rs5993883 polymorphism of the COMT gene was associated with better creative problem-solving skills only in males and not females, indicating an interaction of gender and genotype. In this study, therefore, we looked at a polymorphism in the serotonin transporter gene commonly implicated in heightened stress response, (a 43-base pair deletion in the promoter region of the serotonin transporter gene, SLC6A4; also known as S-allele) and wished to examine if it influenced functional connectivity during creativity under stress. Multiple studies have reported that participants with the S-allele experienced greater negative responses (behavioral, neural and maternal genotype effects on 
offspring) to stress exposure compared to participants without the 43 base pair deletion, or the L-allele (Caspi, A. et al., 2003; Caspi, Hariri, Holmes, Uher, \& Moffitt, 2010; Hariri et al., 2002; Hecht et al., 2016; Kenna et al., 2012; van der Meer et al., 2014; Pezawas et al., 2005; van der Meer et al., 2015; Hariri et al., 2002). With the increased response to stress, there is some preliminary evidence suggesting that in individuals with at least one copy of the S-allele, performance on tasks requiring unconstrained flexibility was significantly impaired under stress (Beversdorf, 2018). It is, therefore, interesting to examine how the presence of this genotype might alter functional connectivity in the brain when performing creativity tasks under stress.

Gender is another factor known to influence stress susceptibility. Studies have reported gender related differences in cortisol secretion levels (Kirschbaum et al., 1992), neural activity (Wang et al., 2007) and cognitive functioning (Lighthall et al., 2012) under stress. A genetic basis has also been proposed for the differential stress susceptibility observed between genders (Dukal et al., 2015). The literature on gender differences in creative tasks is heterogenous with the overall consensus being that while men and women may not differ in terms of their creative ability, the cognitive strategies, and their neural mechanisms that they apply during creative thinking may be distinctly different (Abraham, 2016; Abraham, Thybusch, Pieritz, \& Hermann, 2014). Therefore, gender was an additional factor that we considered in our analysis of how stress affects functional connectivity during creative thinking. 
We hypothesized that stress would negatively impact performance on the CRA task in terms of both number of problems solved and the average time required to solve the problems. We also hypothesized that functional connectivity under stress between the regions of the brain associated with compound remote associates task (such as the left inferior frontal gyrus, left medial frontal gyrus, left middle temporal gyrus, and right superior temporal gyrus (Jung-Beeman, 2005; Jung-Beeman et al., 2004)) would be lower than that under no stress and it would be lower in 1) individuals with at least one copy of the S-allele than individuals homozygous for the L-allele and 2) females compared to males during verbal problem-solving.

\section{MATERIALS AND METHODS}

\section{Participants}

As described previously, a total of forty-five participants (Mean age: $19.5 \pm 1.6$ years) without any significant medical or psychiatric history, including anxiety disorders, were recruited through online advertisements in the University newsletter. All protocols and procedures were approved by the University of Missouri Health Sciences Institutional Review Board. All participants provided written consent to participate in the study.

Genetic Screening and Genotyping 
Participants provided a cheek swab to genotype for the presence of the short (S-allele) or long (L-allele) polymorphism of the serotonin transporter promoter gene. Genotyping was performed using standard methods (Flexigene kit; Qiagen) as detailed elsewhere (Hecht et al., 2016). Individuals with at least one copy of the S-allele were categorized in the S-allele group and those with 1/1 genotype were included in the L-allele group. The presence of the A/G single base substitution on rs25531 (Kenna et al., 2012), T/G single base substitution on rs3813034 (Gyawali et al., 2010) or STin2.10 in Intron 2 VNTR in the 1/1 genotype (Murphy \& Moya, 2011) is known to behave in a less efficient manner like the S-allele. Hence, we ran additional genotyping assessments to look for these substitutions. One participant was regrouped from the L-allele group to the S-allele group based on the presence of the A/G single base substitution on rs25531.

\section{MRI Acquisition and Verbal Problem Solving}

Each participant attended fMRI sessions on two separate days, separated by at least 24 hours. For one half of the participants, the first session was designed to induce stress while the second session was a no stress control session and vice-versa for the other half of the participants. The participants were unaware that one of the sessions had tasks meant to induce stress. They were told that on one day their responses would be timed and performance feedback would be provided while on the other day they did not have any time limit and would not receive any performance feedback. As described previously, participants were asked to refrain from alcohol, nicotine and caffeine for 24 hours prior to the imaging session. Participants also completed the State-Trait Anxiety Inventory 
(STAI) in order to assess baseline anxiety prior to the imaging session and again post imaging session to assess level of anxiety experienced.

During the stress inducing session, the participants were presented with two runs of three iterations of $30 \mathrm{~s}$ blocks of REST-MIST-TASK (Figure 1). During the REST block, the participants were presented a white screen with cross hairs in the center and no other tasks or stimuli were presented. The Montreal Imaging Stress Test (MIST), based off the Trier Social Stress Test (Kirschbaum et al., 1993), consists of several timed mental arithmetic tasks with performance feedback (Dedovic et al., 2005). Based on the practice runs of the subjects, the difficulty of the arithmetic tests was set to give a success rate of $50 \%$. Along with a performance indicator that appeared on their screen, the participants were also provided negative feedback by the experimenter as a social evaluative threat component. When the participants came in for their no stress control session, the MIST block was replaced by simple arithmetic tasks with no time limit or performance feedback. Each TASK block consisted of four Compound Remote Associates (CRA) tasks (Bowden \& Jung-Beeman, 2003) presented for 7 seconds each. The CRA task presented the participants with three prompt words and they had to think of a word that would form a compound word with all three given prompts. (For example: APPLE, CONE, WOOD. Solution: PINE) (Figure 1). Participants practiced the tasks before the start of the imaging session. Number of correct responses and solution latency (subjects were asked to indicate with a button click as soon as they thought of a solution to the problem presented) were recorded offline. 
Figure 3.1 Stimulus protocol. Stimuli were presented in a block design with repeating units of REST-MIST-TASK. The TASK block consisted of prompts for the Compound

Remote Associates (CRA) Task. For example, one of the four prompts for CRA 1 in session 1 were: fish, mine, rush (solution = gold); in session 2 the one of the four prompts for CRA 1 were: illness, bus, computer (solution = terminal). Each block (except for the

REST block at the beginning of the run that was $60 \mathrm{~s}$ long) was $30 \mathrm{~s}$ in duration.

Participants performed two runs of the task within a session and attended two fMRI sessions (separated by at least 24 hours).

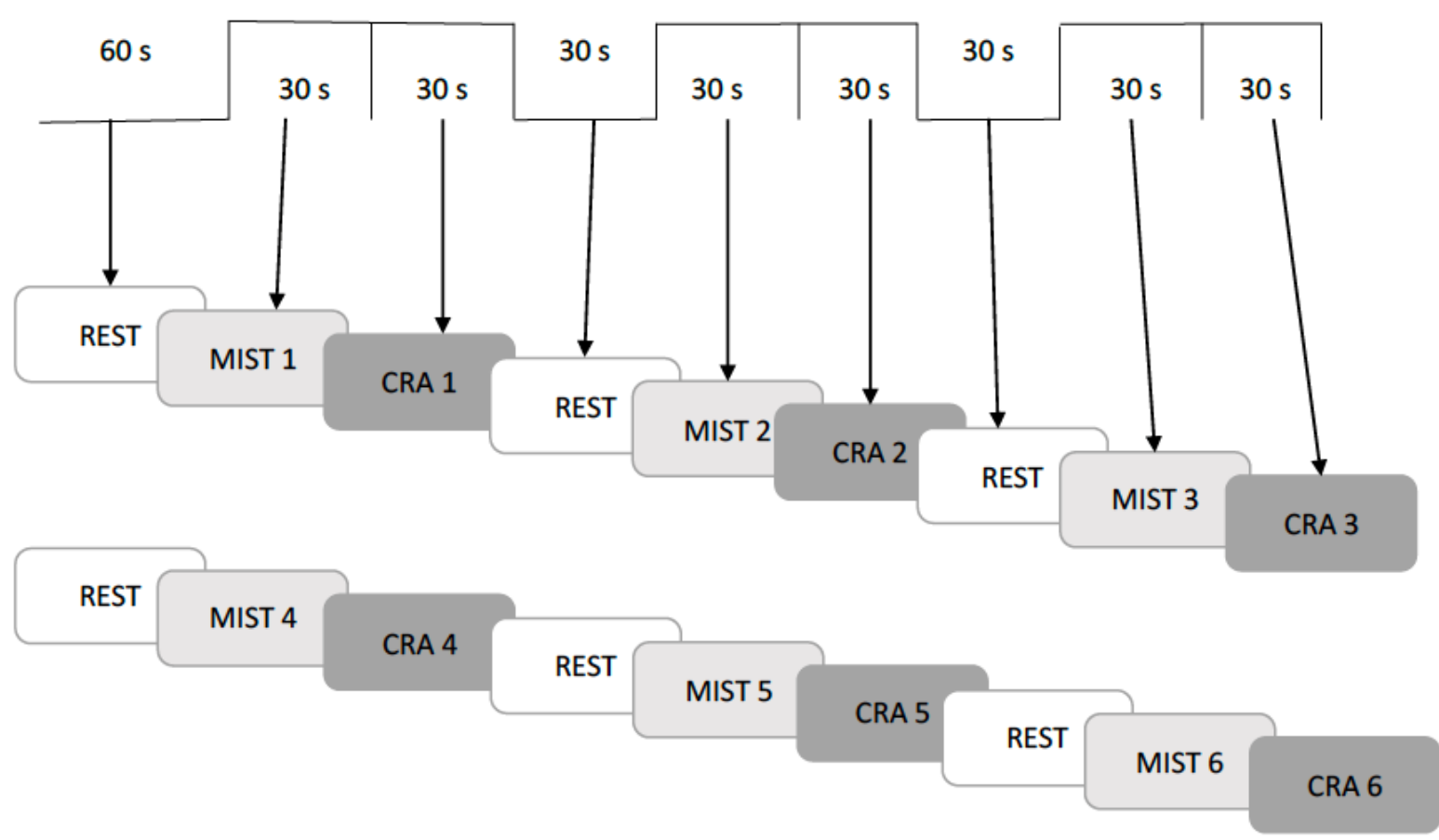

Images were collected on a 3T Siemens Trio Scanner at the University of Missouri Department of Psychological Sciences Brain Imaging Center. Structural T1-weighted images were acquired for anatomical localization (MPRAGE, TR=1920 ms, TE=2.9 ms, Angle $=9$ degrees, FOV/matrix size $=256 \times 256,176$ sagittal slices at $1 \mathrm{~mm}^{3}$ resolution) . 
Functional $\mathrm{T} 2 *$-weighted images were acquired to measure the blood oxygenation level dependent $(\mathrm{BOLD})$ response $\left(\mathrm{EPI}, \mathrm{TR}=2000 \mathrm{~ms}, \mathrm{TE}=30 \mathrm{~ms}\right.$, Flip angle $=90^{\circ}, \mathrm{FOV}=256 \mathrm{x}$ 256, matrix size $=64 \times 64,32$ AC-PC aligned slices at $4 \mathrm{~mm}^{3}$ resolution).

MRI Analysis

FSL (FMRIB Software Library, www.fmrib.ox.ac.uk/fsl) was used to analyze the 4D functional magnetic resonance imaging datasets. The FEAT (FMRI Expert Analysis Tool) Version 6.00 was used to perform preprocessing steps including brain extraction using BET (Brain Extraction Tool) (Smith, 2002), slicing timing correction (interleaved) using Fourier-space time-series phase-shifting, motion correction using MCFLIRT (Motion Correction FLIRT) (Jenkinson et al., 2002), Gaussian spatial smoothing (FWHM of $5 \mathrm{~mm}$ ), high pass temporal filtering (Gaussian-weighted least-squares straight line fitting, cutoff $100 \mathrm{~s}$ ), grand-mean intensity normalization of the entire 4D dataset and registration of each participant's functional images to the respective high resolution structural image and standardized MNI space (using FLIRT (FMRIB's Linear Image Registration Tool)). Relative motion greater than $2 \mathrm{~mm}$ along either of the $\mathrm{x}-$, $\mathrm{y}-\mathrm{or} \mathrm{z}-$ axis or any of the rotational axes led to exclusion from further analysis. Gaussianised T or $\mathrm{Z}$ static images were thresholded using clusters determined by $\mathrm{Z}>2.3$ and a (corrected) cluster significance threshold of $p=0.05$.

The Harvard - Oxford Cortical Atlas in FSL was used to create individualized masks for the regions of interest for each subject. The regions of interest explored were the left 
inferior frontal gyrus, left middle frontal gyrus, left middle temporal gyrus, left parietal lobe, left fusiform gyrus, left posterior cingulate gyrus and the right superior temporal gyrus due to their known involvement while performing CRA tasks (Jung-Beeman, 2005; Jung-Beeman et al., 2004) (Figure 2). The FEATQUERY tool of FSL was used to extract a) percentage signal change in BOLD signal (with respect to rest) and b) time series of voxel of maximum activation within each ROI during the task block for each subject. The percentage signal change in BOLD signal was used to examine effects of stress on activation within each ROI that might potentially influence functional connectivity. For functional connectivity analyses, time series extracted from within task blocks were used to account for the effect of activation magnitude differences between stress conditions on FC. Bivariate correlations were performed between the time series for each of the ROI pairs. The resulting correlation coefficients were converted using Fisher's r-to-ztransformation providing a z-score measure of functional connectivity strength. In order to determine changes in overall functional connectivity strength across all ROIs under the influence of stress, z-scores of correlation coefficients across all ROIs were computed for each individual subject under stress and no stress.

Figure 3.2 Regions of interest. The a priori regions of interest explored were (a) left inferior frontal gyrus (red), left middle frontal gyrus (yellow), left middle temporal gyrus (green), left parietal lobe (purple), (b) right superior temporal gyrus (orange), (c) left posterior cingulate gyrus (pink) and (d) left fusiform gyrus (blue). Masks centered on the a priori regions of interest were created using the Harvard - Oxford cortical atlas in FSL and used to analyze differences in activation and functional connectivity while performing creative tasks under stress and no stress. 

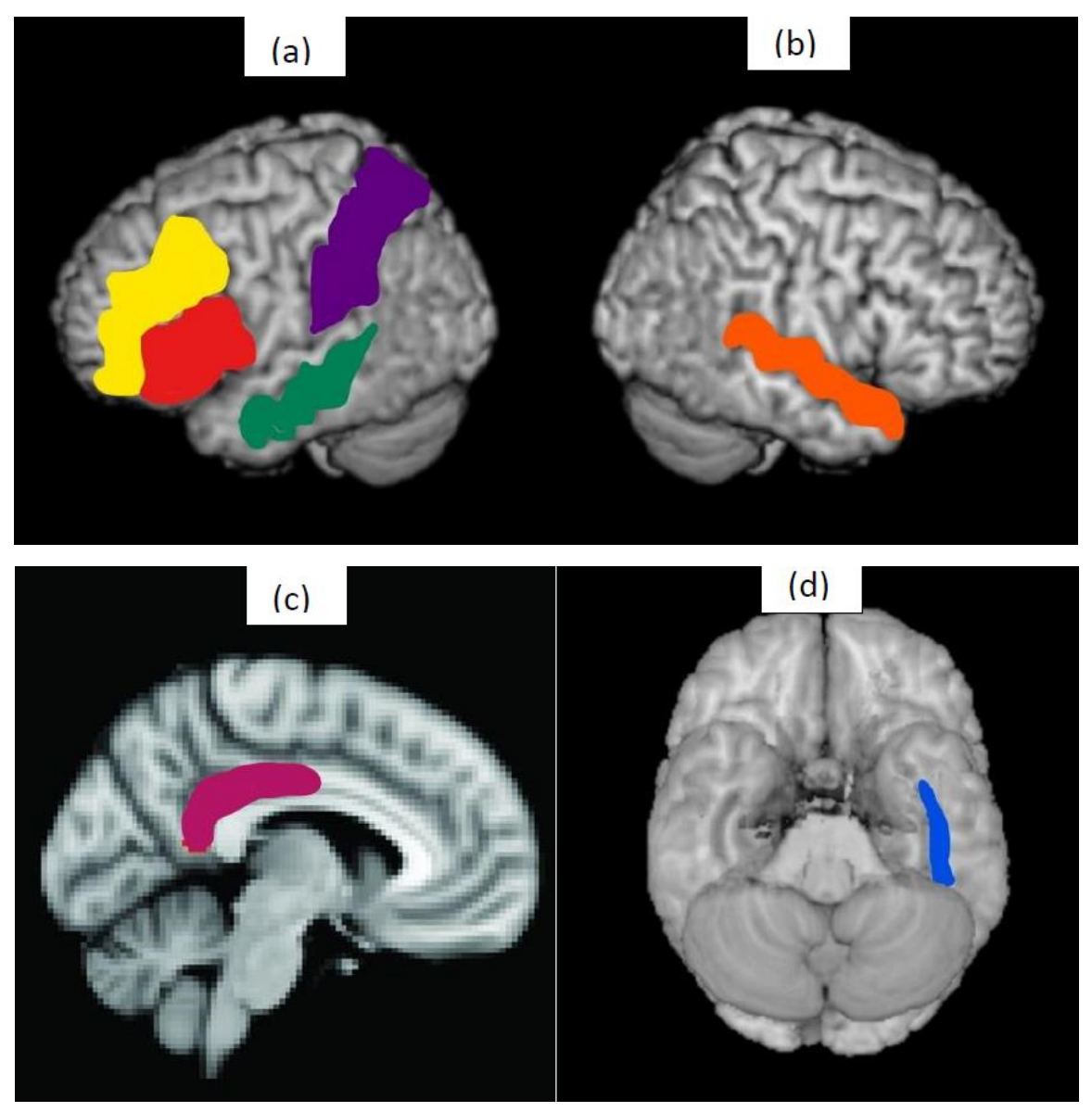

Statistical Analysis

A series of repeated measures multivariate analysis of variance (MANOVA) tests (SPSS ver 24, IBM Corp.) were carried out to examine: (1) performance on tasks: (2(stress, no stress)*2(score stress, score no stress)), (2(stress, no stress)*2(solution latency stress, solution latency no stress), (2) activation in the regions of interest: (2(stress, no stress)*7 (ROIs)), (3) average functional connectivity across ROIs: (2-way MANOVA, (stress, no stress)*overall functional connectivity) and (4) functional connectivity strength between individual ROI pairs: (2 (stress, no stress)*21 (ROI pairs)). The analyses were performed 
again including gender and genotype as between subject factors. Bivariate correlations were performed between task performance measures and functional connectivity strengths between the regions under stress and no stress in order to understand the effects of functional connectivity strength on task performance. Bonferroni corrections for multiple comparisons were applied to all analyses and the results reported here survived correction.

\section{RESULTS}

Demographics

Functional magnetic resonance imaging data from 32 participants was analyzed (5 participants were dropped from the study because they did not attend the second session and data from 8 participants was not included in further analysis due to excessive motion (exceeding $2 \mathrm{~mm}$ along either the linear or rotational axes as mentioned above) within the scanner). Of the 32 participants included in the final analysis, there were 12 male participants and 15 participants had the S-allele. (Table 3.1).

\section{Table 3.1 Demographics of participants}




\begin{tabular}{|c|c|c|c|c|c|c|c|c|c|}
\hline & $\begin{array}{c}\text { Total } \\
(\mathrm{n}=32)\end{array}$ & Male & Female & $\mathrm{X}^{2}$ & $\mathrm{p}$ & $\begin{array}{l}\text { S-allele } \\
(n=15)\end{array}$ & $\begin{array}{c}\text { L-allele } \\
(\mathrm{n}=17)\end{array}$ & $\mathrm{X}^{2}$ & $\mathrm{P}$ \\
\hline $\begin{array}{c}\text { AGE } \\
\text { (YEARS) }\end{array}$ & $\begin{array}{l}19.9 \\
(1.6)\end{array}$ & $\begin{array}{l}19.7 \\
(2.0)\end{array}$ & $\begin{array}{l}20.1 \\
(1.5)\end{array}$ & 10.72 & 0.097 & $\begin{array}{l}19.9 \\
(1.2)\end{array}$ & $\begin{array}{l}20.1 \\
(2.0)\end{array}$ & 8.44 & 0.208 \\
\hline $\begin{array}{c}\text { GENDER } \\
(\mathrm{M} / \mathrm{F})\end{array}$ & $12 / 24$ & - & - & - & - & $5 / 10$ & $6 / 11$ & - & - \\
\hline
\end{tabular}

GROUP COMPARISONS WERE PERFORMED USING CHI-SQUARE TEST. ALL VALUES ARE SHOWN AS MEAN(SD).

M=MALE, F=FEMALE

STAI scores pre and post the imaging session were not significantly different as reported earlier (Chapter 2, Results: Demographics).

\section{Verbal Problem Solving}

A paired samples t-test revealed that there was no significant change in number of problems solved under stress $(\operatorname{Mean}(\mathrm{SD})=9.0(3.7))$ and no stress $($ Mean $(\mathrm{SD})=8.9(3.8))$ $(\mathrm{t}=0.05, p=0.9)$. The effect of stress on solution latency was also evaluated. When response times for only correct responses (excluding response times for button clicks with incorrect responses and button clicks where the participants later indicated that they did not know the solution to the problem) were analyzed, a stress $\mathrm{x}$ genotype interaction effect trending towards significance was observed $(F(1,23)=7.260, p=0.06)$. While no significant differences between stress and no stress were observed in either genotype in post hoc t-tests, the trend appeared to be driven by nonsignificant effects in opposite directions across genotypes. (Figure 3.3). 
Figure 3.3 Verbal problem solving. A stress x genotype interaction effect trending towards significance was observed $(F(1,23)=7.260, p=0.06)$ for solution latency on only problems where correct responses were recorded. Post hoc t-tests did not reveal significant differences between individual groups but $\mathrm{S}$-allele subjects appear to display greater latency of response under stress compared to no stress.

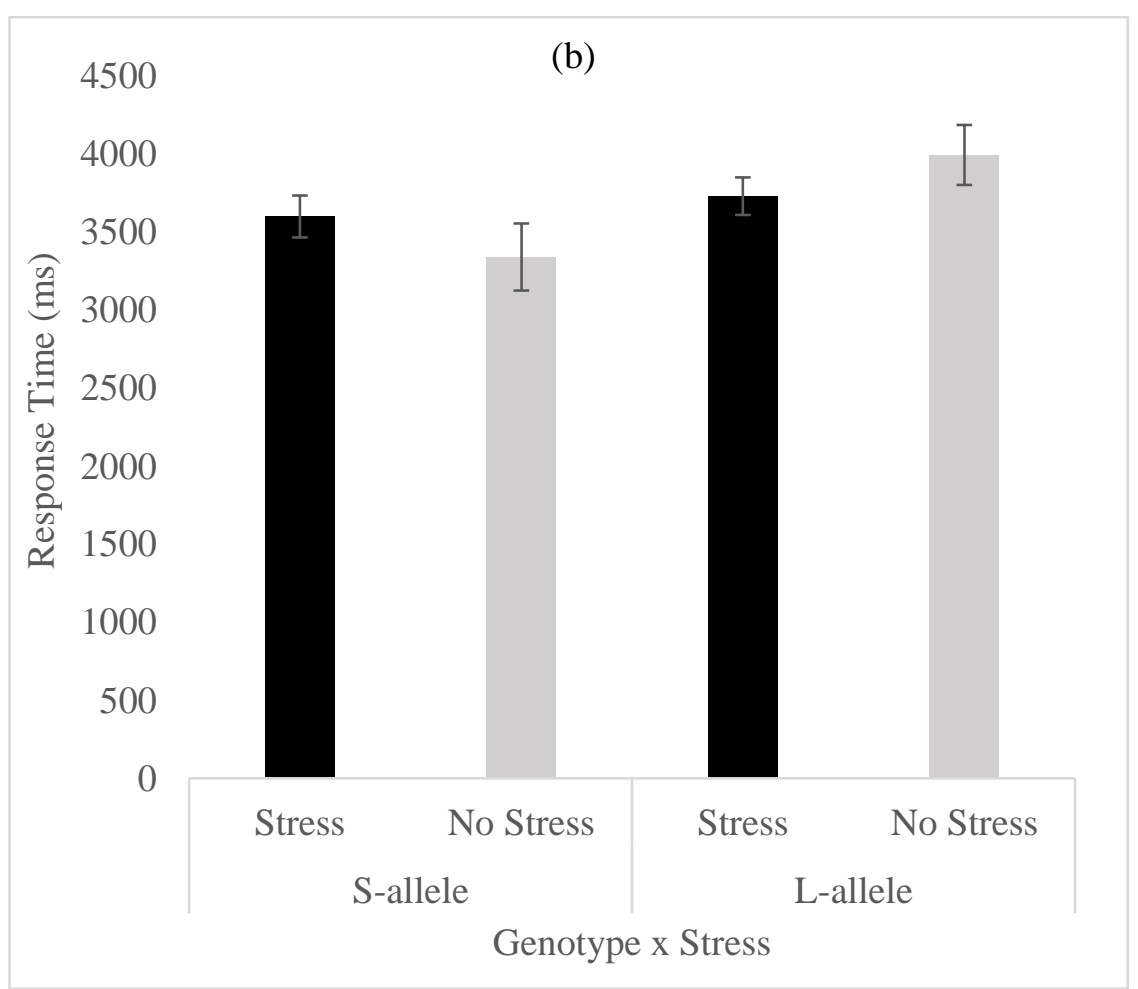

ROI activation and Functional Connectivity

Figure 3.4 displays the group activation maps for participants $(n=32)$ while completing the Compound Remote Associates task under (a) stress and (b) no stress.

Figure 3.4 Group activation maps. Group activation maps for participants $(n=32)$ performing the Compound Remote Associates task under (a) stress and (b) no stress. 
(a) Thresholded activation images

2.3

5.4

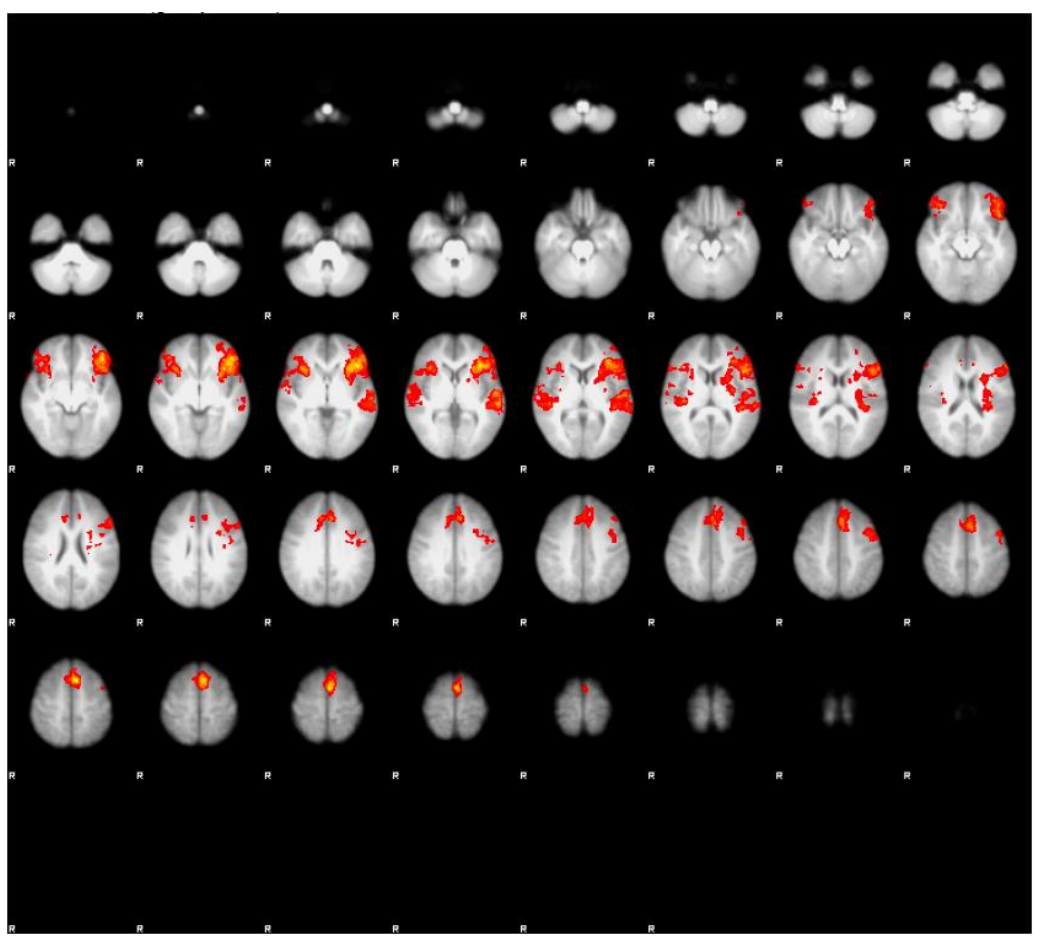

(b) Thresholded activation images

2.3

5.6

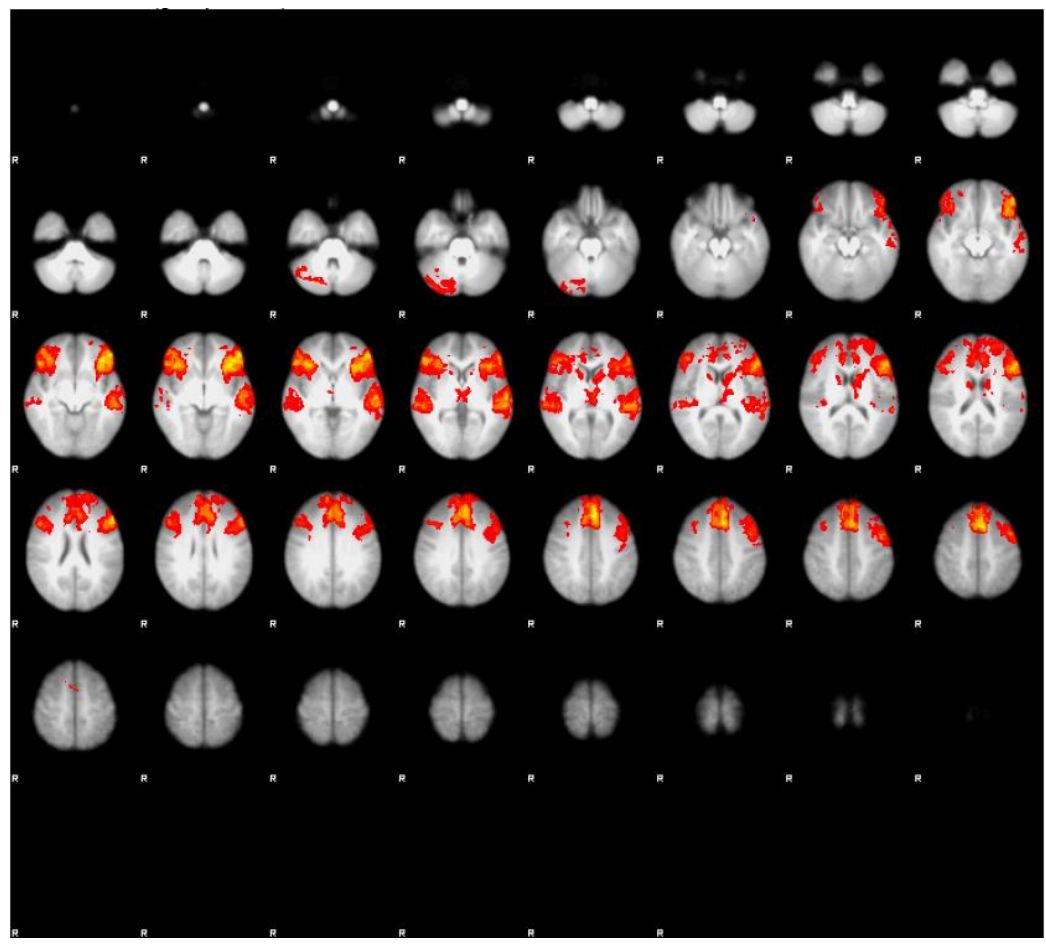


A repeated measures MANOVA did not reveal a significant effect of stress $(F(1,28)=0.01, p=0.91)$, stress $\mathrm{x}$ gender $(F(1,28)=1.49, p=0.23)$ or stress $\mathrm{x}$ genotype $(F(1,28)=0.11, p=0.74)$ on activation across the ROIs. On Bonferroni corrected assessment of individual ROIs, in case of focal effects, the only finding was a significant stress $\mathrm{x}$ gender interaction effect observed on percentage signal change in the right superior temporal gyrus $(F(1,28)=4.49, p=0.04)$ (Figure 3.5). Post hoc t-tests revealed that males showed a significant increase in percentage signal change in the region while performing the CRA task under stress compared to females. We did not observe any other significant changes in percentage signal change under stress and no stress in any of the other regions of interest we looked at.

Figure 3.5 ROI activation. A significant stress $x$ gender interaction effect was observed on activation in the right superior temporal gyrus $(F(1,28)=4.49, p=0.04)$. Post hoc t-tests showed that males had increased activation in the region while attempting creative tasks than females $(t=2.22, p=0.033)$. 


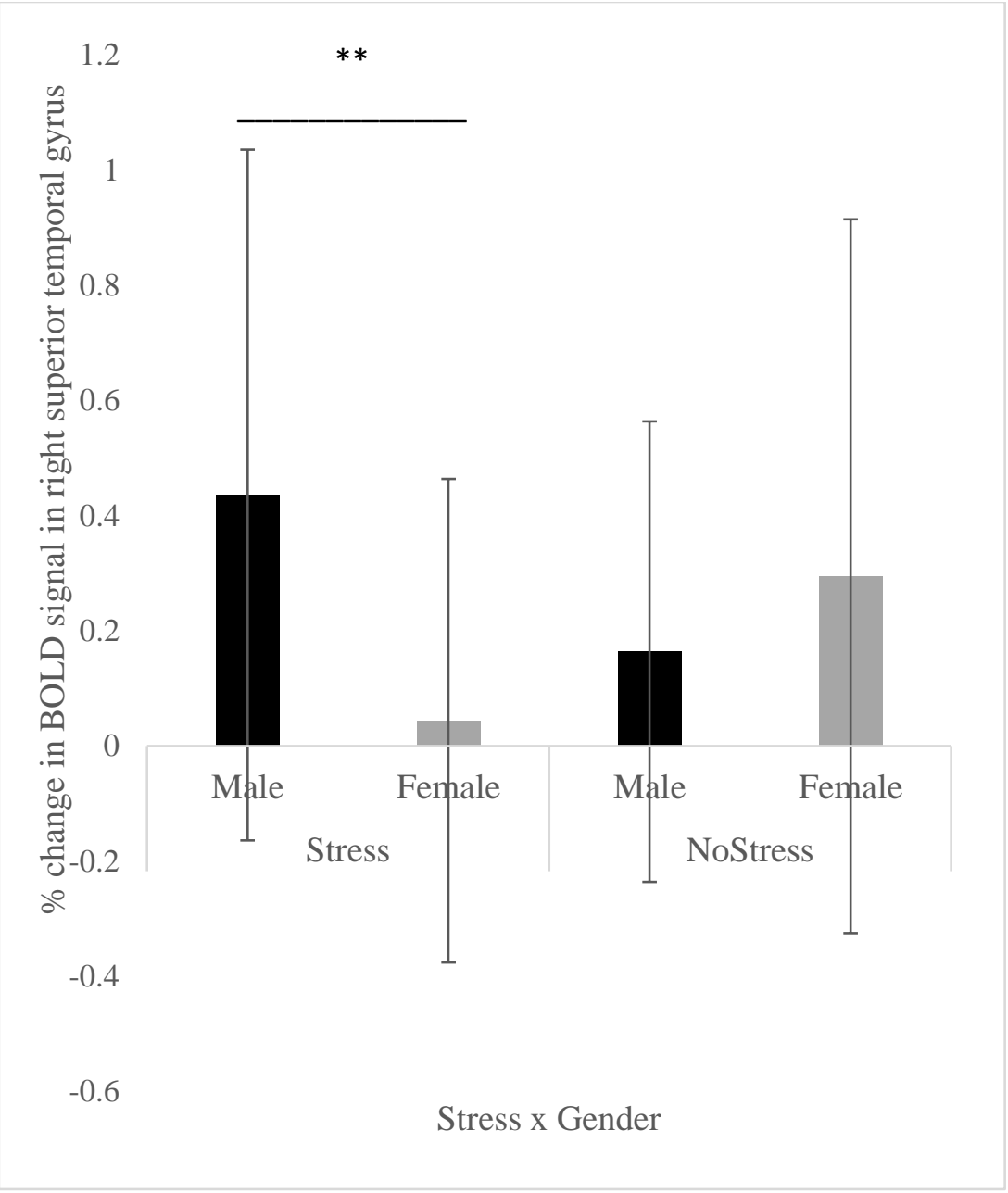

There were no significant effects of stress $(F(1,28)=0.43, p=0.51)$ or stress $\mathrm{x}$ gender $(F(1,28)=0.41, p=0.53)$ and stress $\mathrm{x}$ genotype $(F(1,28)=0.007, p=0.93)$ interactions on overall functional connectivity across all ROIs. The possible effects on individual ROIs pairs was of interest and therefore, we ran repeated measures MANOVAs for each individual pair, with Bonferroni corrections for multiple comparisons.

A three-way interaction effect for stress $x$ gender $x$ genotype was observed on the functional connectivity strength between the left inferior frontal gyrus and the left middle temporal gyrus $(F(1,28)=5.26, p=0.03)$. Post hoc $\mathrm{t}$-tests revealed that males with the $\mathrm{S}$ - 
allele displayed significantly lower strength of functional connectivity between the regions when attempting verbal problem solving under stress compared to both S-allele females $(\mathrm{t}=-2.418, p=0.03)$ and L-allele males $(t=-3.44, p=0.007)$. L-allele males were found to have significantly greater strength of functional connectivity between the regions than L-allele females when verbal problem solving under stress $(t=2.209, p=0.04)$ (Figure 3.6). No significant differences were observed between the individual groups for the stress vs. no stress comparison.

Figure 3.6 Functional connectivity results. A three way interaction effect for stress $x$ gender $\mathrm{x}$ allele was observed on functional connectivity between the left inferior frontal gyrus and the left middle temporal gyrus $(F(1,28)=5.26, p=0.03)$. Post hoc t-tests revealed that $S$-allele males had reduced strength of functional connectivity compared to S-allele females $(t=-2.418, p=0.03)$ and L-allele males $(t=-3.44, p=0.007)$ during creativity task under stress. L-allele males were found to have significantly greater strength of functional connectivity between the regions than L-allele females when verbal problem solving under stress $(t=2.209, p=0.04)$. 


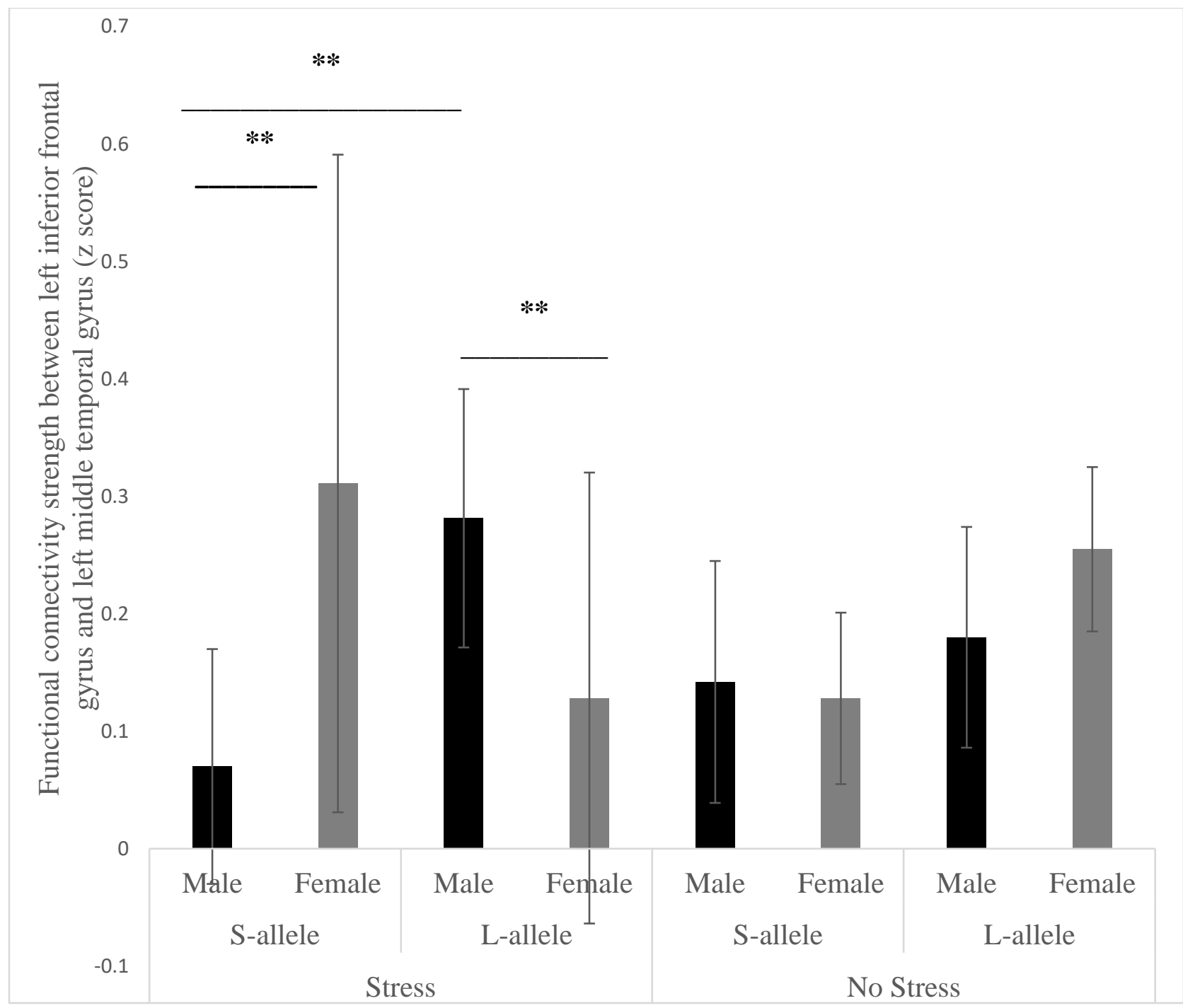

Activation, functional connectivity and task performance

In order to examine the effect of neural activation and functional connectivity strength changes associated with stress on verbal problem solving, we ran bivariate correlations of neural activation and functional connectivity strength with scores and solution latency on the compound remote associate task. Though a stress $\mathrm{x}$ gender interaction effect was observed on activation in the right superior temporal gyrus, no significant correlations 
with solution latency under stress and no stress were observed in this region. However, number of problems solved under no stress was found to be significantly correlated with activation in the right superior temporal gyrus under no stress $(r=0.405, p=0.02)$. In order to determine if change in performance induced by stress in solution latency was related to changes in functional connectivity markers, we ran bivariate correlations between difference in solution latency under stress and no stress and a) overall difference in functional connectivity under stress and no stress and b) differences in functional connectivity in individual ROI pairs under stress and no stress. Differences in overall functional connectivity were not found to be associated with changes in solution latency under stress and no stress. However, for S-allele participants, we observed that differences in solution latency under stress and no stress were strongly correlated to change in functional connectivity strength between the left middle temporal gyrus and the left posterior cingulate gyrus under stress and no stress $(r=0.653, p=0.029)$. For L-allele participants, changes in solution latency showed a strong negative correlation with differences in functional connectivity strength between the left inferior frontal gyrus and left middle temporal gyrus under stress and no stress $(r=-0.565, p=0.035)$.

\section{DISCUSSION}

This study intended to look at the neural correlates of the effects of stress on convergent verbal problem-solving tasks related to creativity. The compound remote associates test was used a measure of cognitive flexibility and the Montreal Imaging Stress Test was used to induce stress in subjects while undergoing fMRI imaging. 
Stress is known to impair performance on tasks requiring flexible access to remote, distributed networks due to heightened arousal and narrowed focus (Martindale \& Greenough, 1973). As a result, stress has a direct effect on the neural substrates of tasks that require coming up with creative solutions overriding possibly more dominant solution pathways.

Regarding functional activation differences, there was no main effect of stress and the only isolated finding observed across ROIs was increased mean activation in the right superior temporal gyrus in males compared to females in response to verbal problem solving under stress. Previous studies have already reported on the role of the right hemisphere in insight based problem solving, shedding light on its role in maintaining solution related activation to problems that are yet to be solved (Bowden \& Jung-beeman, 2003; Zhao et al., 2014). It is possible that the heightened response in the region observed under stress in males here is reflective of either the additional load from the stressor immediately preceding the task or an increased tendency to linger on unsolved problems in males with the right superior temporal gyrus continually engaged in figuring out solutions to previously presented problems.

The results from our functional connectivity analyses reveal that males and females differ in how genotype affects the imaging response to stress during cognitive flexibility tasks. Notably, while performing a task requiring cognitive flexibility under stress, males with the S-allele displayed decreased strength of functional connectivity between the left 
inferior frontal gyrus and the left middle temporal gyrus compared to both S-allele females and L-allele males under stress. L-males however, displayed greater strength of functional connectivity between the regions under stress compared to L-allele females. While part of this result is consistent with our hypothesis that participants with the Sallele would have decreased strength of functional connectivity during creative problem solving under stress, it also furthers our understanding by pointing out that this decrease was gender specific. Interestingly, the functional connection between the left inferior frontal gyrus and the left middle temporal gyrus displayed a decrease in strength of connectivity under stress for S-allele males compared to both S-allele females and Lallele males. A potential explanation for this finding could be that while creative thinking does require access to remote, non-dominant pathways in the brain, there may be variability in some form of top-down attentional control that is exerted to ensure that the solutions thought of are consistent with the problem presented and the mind is not just "wandering" (Beaty et al., 2014). In S-allele males, stress potentially leads to a greater reduction in this frontal control compared to S-allele females or L-allele males.

We did not observe differences in number of CRA tasks solved by the participants under stress and no stress. Solution latency could potentially be more sensitive and hence, was examined. The time taken to record only correct responses under stress and no stress, with Bonferroni correction for multiple comparisons, showed a trend towards significance in a genotype specific manner, possibly limited by the modest set of problems used in the MRI. The interaction trend observed, nevertheless, was in the same direction as has been noted in a previous study (Beversdorf et al., 2017). Additionally, 
genotype was also found to influence the association between changes in solution latency and changes in functional connectivity strength between specific brain regions under stress and no stress. This further supports the complex interaction of genotype and imaging markers in determining stress susceptibility in tasks requiring cognitive flexibility.

These results show that stress affects activation and functional connectivity during creative problem solving in a gender and genotype specific manner. This begins to elucidate the finer neural underpinnings of creativity and the interplay of factors that influence creativity. This has implications in not only understanding creativity in the general population but also understanding a possible link between creativity and psychopathology (Heilman et al., 2003). Highly creative people have been documented to suffer from depression, bipolar disorder and/or addiction problems. People with schizophrenia are known to perform well on insight tasks of creativity (Karimi, Windmann, Güntürkün, \& Abraham, 2007). These observations have been tied to alterations in catecholamines, such as a reduction in dopamine. It is also possible that stress, and its effect on the neural correlates of cognitive flexibility mediated via catecholamines, might have a role to play in shifting from a normal creative thought process to the development of the pathology. This may offer the opportunity for neuropharmacological modulation of these conditions. Future studies will want to explore interventions targeting cognitive flexibility under unfavorable conditions, such as stress, while paying attention to potentially influential factors such as genetics and gender. Additionally, a number of other factors, such as the influence of the dopamine system and 
its associated genes (Reuter, Roth, Holve, \& Hennig, 2006), norepinephrine system and its modulation using pharmacological intervention (J. K. Alexander et al., 2007), along with different types of creativity other than convergent tasks (Beversdorf, 2018) need to be assessed in future analysis to get a more complete understanding of how stress modulates functional connectivity in the brain associated with cognitive flexibility in healthy as well as clinical populations.

\section{LIMITATIONS}

There are some limitations that must be taken into consideration while interpreting the results of this study. For one, the number of male participants was small. The three-way interaction results must, therefore, be interpreted with caution due to the small sample size. While this study focused on regions of the brain known to be involved in CRA tasks, it is possible that stress may have altered the functional activation in and connectivity between other regions of the brain that might have a regulatory role. Future studies will need to look at other potential regions as well. Genotype specific trends in differences in solution latency under stress and no stress were observed, driven possibly by nonsignificant effects in opposite directions across genotypes, limited by the smaller number of tasks administered in the imaging environment. We also observed differences in the neural underpinnings of cognitive flexibility. The MIST paradigm has been previously shown to successful induce stress, as reflected through changes in cortisol levels (Dedovic, Aguiar, et al., 2009; J. C. Pruessner et al., 2008), however, reliable cortisol levels were not available in this study to reaffirm the induction of stress. 


\section{SUMMARY}

The current study looked at the influence of stress, gender and genotype on functional connectivity during verbal problem solving as a measure of creative thinking. The functional connection between the left inferior frontal gyrus and the left middle temporal gyrus was the one found to be impaired under stress in S-allele males compared to both S-allele females and S-allele males. This leads to a better understanding of the factors influencing neural processes that are involved in creative thinking, thereby enabling interventions to optimize flexible thinking and performance even in stressful situations. 


\section{CHAPTER FOUR:}

\section{AMYGDALA-FUSIFORM FUNCTIONAL CONNECTIVITY RESPONSE TO EMOTIONAL FACES UNDER STRESS}

\section{INTRODUCTION}

The amygdala is a central component of the fear and emotional neural circuitry in humans and as such plays an important role in triggering the "fight or flight" response basic to survival of the species (LeDoux, 2007). It is, therefore, not surprising at all that amygdala dysfunction is observed in several neuropsychiatric conditions such as phobias (Kim et al., 2011), anxiety (Roy et al., 2010), depression (Pezawas, Meyer-lindenberg, et al., 2005), posttraumatic stress disorder (Rabinak et al., 2011; Rauch et al., 2000; Sripada et al., 2012; Stevens et al., 2013), paranoid schizophrenia (Williams et al., 2004) and autism spectrum disorders (Baron-Cohen et al., 2000; Dziobek, Bahnemann, Convit, \& Heekeren, 2010; Kleinhans, N. M., Richards, T., Weaver, K., Johnson, L. C., Greenson, J. et al., 2010; Kliemann, Dziobek, Hatri, Baudewig, \& Heekeren, 2012; Schultz, 2005; Sweeten, Posey, Shekhar, \& McDougle, 2002). Associated with its role in emotionality, the amygdala is also involved in recognition of facial emotions working in tandem with the fusiform gyrus (the region of the brain specific to identifying faces) (Herrington, Taylor, Grupe, Curby, \& Schultz, 2011). Further evidence for the synergistic activity of the amygdala and fusiform gyrus comes from a study by Vuilleumier, Richardson, Armony, Driver, \& Dolan (2004). This study reported that in patients with amygdala lesions, the fusiform gyrus response to fearful faces was significantly attenuated 
compared to healthy controls and patients with lesions in the hippocampus. Moreover, the degree of amygdala damage was found to be related to the observed activation in the fusiform gyrus. A neuromodulatory role for the amygdala in processing emotional faces has also been proposed according to which the amygdala directs more attention towards fearful faces that indicate the presence of a possible threat but do not give information on the source of the threat (Morris et al., 1998). A dysfunction in amygdala and fusiform functional connectivity could potentially contribute towards the inability to identify emotions as observed in schizophrenia (Feinberg, Rifkin, Schaffer, \& Walker, 1986; Gur et al., 2002) and autism (Dziobek et al., 2010; Hadjikhani et al., 2004; Kliemann et al., 2012) or hyperreactivity to emotional faces characteristic of posttraumatic stress disorder (Rauch et al., 2000).

Stress is a factor known to affect activity in and functional connectivity of the amygdala. Pruessner et al. (2008) showed that acute stress was associated with extensive deactivation of the limbic system structures, including the amygdala. In a different study, amygdala functional connectivity with cortical midline structures was found to be upregulated following exposure to acute psychosocial stress (Veer et al., 2011). Acute stress has also been shown to upregulate activity in both the amygdala and the fusiform gyrus in response to emotional stimuli embedded in stressful videos (Hein J F van Marle, Hermans, Qin, \& Fernández, 2009). Generally, stress is associated with a state of hypervigilance and narrowed attention, driven by increased noradrenergic tone, which in turn also has an effect on amygdala activity (Onur et al., 2009; Van Stegeren et al., 2005). Therefore, it is of interest to explore whether this state of hypervigilance upregulates 
activity in the amygdala and its functional connection to the fusiform gyrus such that there is increased attention towards the emotional face stimulus.

Another important factor that is known to influence not just amygdala activation, but also stress reactivity of the amygdala is the presence of the short allele (S-allele) of the serotonin transporter gene (SLC6A4) polymorphism (N. Alexander et al., 2012; Dannlowski et al., 2010; Drabant et al., 2012; Hariri, Drabant, \& Weinberger, 2006; Kempton et al., 2009; Kobiella et al., 2011; Kruschwitz et al., 2015; Lesch et al., 1996; Pezawas, Meyer-Lindenberg, et al., 2005; Smolka, 2005). The short allele consists of a 43 base pair deletion on the promoter region of the gene (5HTTLPR) (Heils et al., 1996). The resultant transporter protein is about $50 \%$ less efficient than that found in individuals without the 43-base pair deletion - the long allele (L-allele). A study by (Hariri et al., 2002) showed that individuals with the S-allele showed an approximate fivefold increase in activation when viewing emotional faces compared to individuals with the L-allele under stress. Also, neuroticism in individuals with the S-allele genotype has been specifically linked to resting state connectivity between the amygdala and the fusiform gyrus (Kruschwitz et al., 2015). Therefore, we were interested in how this genotype might influence amygdala - fusiform gyrus functional connectivity under stress while viewing emotional faces.

Additionally, the role of gender in this interaction was of interest. Studies have demonstrated that there are differences in stress reactivity (Kirschbaum et al., 1992) and neural response to emotional stimuli (Wrase et al., 2003) between males and females. 
Therefore, we explored a potential role for gender in upregulating the connectivity between the amygdala and fusiform gyrus when viewing emotional faces under stress. There are studies showing that females may be more susceptible to depression than males (Kessler et al., 1993) and that the short allele of the serotonin transporter gene may significantly influence this effect (Dukal et al., 2015). We additionally explored if genotype interacted with gender in influencing the upregulation of amygdala-fusiform connectivity under stress.

Based on previous literature, we hypothesized that stress would result in upregulated functional connectivity between the amygdala and the fusiform gyrus and that this upregulation would be greater in individuals with the S-allele and especially in females with the S-allele.

\section{MATERIALS AND METHODS}

\section{Participants}

A total of forty-five participants (Mean age: $19.5 \pm 1.6$ years) without any significant medical or psychiatric history, including anxiety disorders, were recruited through online advertisements in the University newsletter. All protocols and procedures were approved by the University of Missouri Health Sciences Institutional Review Board. All participants provided written consent to participate in the study. 


\section{Genetic Screening and Genotyping}

Standard methods, as described elsewhere (Hecht et al., 2016), were used to genotype for the presence of the short allele (S-allele) or long allele (L-allele) of the serotonin transporter gene polymorphisms from buccal swabs of each participant. Participants with at least one copy of the short allele (s/s or s/l) were grouped in the $S$-allele category and participants with two copies of the long allele (1/l) were placed in the L-allele category. Additional analyses were performed to detect presence of $A / G$ single base substitution on rs 25531, T/G single base substitution on rs3813034 or STin2.10 in Intron 2 VNTR in the 1/1 genotype. The presence of these substitutions are known to render the serotonin transporter gene less efficient in a manner similar to the functioning of the S-allele. One participant with the $1 / 1$ genotype had the $\mathrm{A} / \mathrm{G}$ single base substitution on rs25531 and was grouped with the S-allele participants for all subsequent analyses.

\section{MRI Acquisition}

Each participant attended fMRI sessions on two separate days, separated by at least 24 hours. For one half of the participants, the first session was designed to induce stress while the second session was a no stress control session and vice-versa for the other half of the participants. The participants were unaware that one of the sessions had tasks meant to induce stress. They were told that on one day their responses would be timed and performance feedback would be provided while on the other day they did not have 
any time limit and would not receive any performance feedback. All participants were debriefed at the end of the study about the stress induction.

During the stress inducing session, the participants were presented with six repetitions of REST-MIST-TASK block paradigm. During the REST block, the participants viewed a white screen with cross hairs in the center and no additional instructions or tasks were provided. During the Montreal Imaging Stress Test (MIST) (Dedovic et al., 2005) block, several timed mental arithmetic tasks (with a success rate $50 \%$ based on practice runs) were presented. These were accompanied by a performance feedback indicator on the screen as well as negative feedback from the experimenter, which served as social evaluative threat components. During the no stress control session, the MIST block was replaced by simpler mental arithmetic tasks with no time limits and no feedback. The REST and MIST blocks were 30 seconds each. The TASK block consisted of viewing a triad of faces, obtained from the International Affective Picture System (IAPS) (Libkuman, Otani, Kern, Viger, \& Novak, 2007), in quick succession of which the image at the top of the triad (target image; either an emotional face or a neutral one) matched with one of the two images at the bottom. In order to ensure that the subjects were paying attention to the images, they were asked to indicate via button press which image (left or right) at the bottom matched with the image at the top (Figure 4.1). Each TASK block was 48 seconds in duration.

Fig 4.1 Sample image used for the Faces task. Participants were required to indicate via button press which of the two pictures at the bottom (left or right) matched with the one 
at the top. All images were acquired from the International Affective Picture System (IAPS).
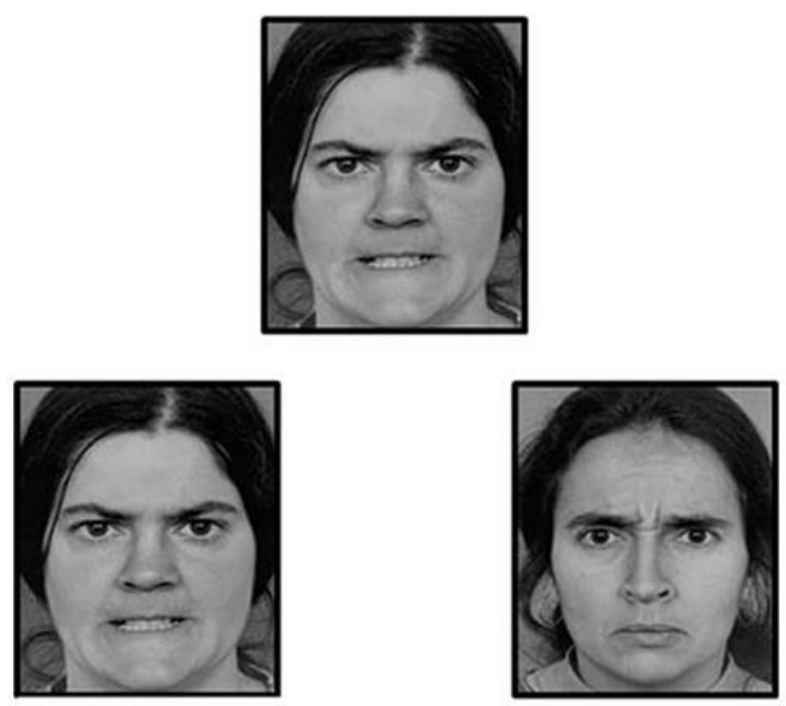

Images were collected on a 3T Siemens Trio Scanner at the University of Missouri Department of Psychological Sciences Brain Imaging Center. Structural T1-weighted images were acquired for anatomical localization (MPRAGE, TR=1920 ms, TE=2.9 ms, Angle $=9$ degrees, FOV/matrix size $=256 \times 256,176$ sagittal slices at $1 \mathrm{~mm}^{3}$ resolution) . Functional $\mathrm{T} 2 *$-weighted images were acquired to measure the blood oxygenation level dependent $(\mathrm{BOLD})$ response $\left(\mathrm{EPI}, \mathrm{TR}=2000 \mathrm{~ms}, \mathrm{TE}=30 \mathrm{~ms}\right.$, Flip angle $=90^{\circ}, \mathrm{FOV}=256 \mathrm{x}$ 256, matrix size $=64 \times 64,32 \mathrm{AC}-\mathrm{PC}$ aligned slices at $4 \mathrm{~mm}^{3}$ resolution).

MRI Analysis 
The 4D functional magnetic resonance images acquired were analyzed using the FMRIB Software Library (FSL, www.fmrib.ox.ac.uk/fsl). Standard preprocessing steps were performed using the FMRI Expert Analysis Tool (FEAT) Version 6.00. The Brain Extraction Tool (BET) was used to extract and separate brain matter from surrounding skull and neck regions. Fourier-space time-series phase-shifting was used to correct for timing in interleaved acquisition. Each participant's functional image was first registered on to the respective high resolution structural image and then onto a standard MNI template using FMRIB's Linear Image Registration Tool (FLIRT). Motion correction was applied using Motion Correction FLIRT (MCFLIRT) and participants who had relative motion more than $2 \mathrm{~mm}$ along any of the linear $(\mathrm{x}, \mathrm{y}, \mathrm{z})$ or rotational axes (roll, pitch, yaw) were excluded from further analyses. Gaussian spatial smoothing with FWHM 5mm, Gaussian-weighted least-squares straight line fitting high pass temporal filtering with cutoff $100 \mathrm{~s}$ and grand mean normalization of the entire data set were also performed as part for preprocessing. The specific time blocks where the participants were viewing emotional faces were used in the general linear model. The clusters in the resulting Gaussianised $\mathrm{T}$ or $\mathrm{Z}$ static images were thresholded at $\mathrm{Z}>2.3$ with corrected cluster significance threshold, $\mathrm{p}=0.05$.

Since the aim of the study was to examine connectivity between the amygdala and fusiform gyrus while viewing emotional faces under conditions of stress and no stress, the Harvard Oxford Subcortical Atlas, part of the FSL software, was used to generate masks for the left amygdala, right amygdala, left fusiform gyrus and right fusiform gyrus (Figure 4.2). Using the FEATQUERY tool of FSL, the percentage change in BOLD 
signal with respect to rest in the regions of interest was extracted. The activations in the regions of interest were examined due to their potential influence on functional connectivity. The time series of the voxel of maximum activation within each ROI was also extracted for each subject to compute functional connectivity. The time series were extracted from within the task blocks to account for the effect of activation magnitude differences between stress conditions on functional connectivity. Fisher's r-to-z transformation was performed on the correlation coefficients obtained by carrying out bivariate correlations on the time series of each of the ROI pairs. The resulting z-score was used as a measure of functional connectivity strength as has been done previously (Tivarus et al., 2008).

Figure 4.2 Regions of interest created using the Harvard-Oxford Cortical Structural Atlas tool in FSL. (a) Red: Left and right amygdala (b) Blue: Left and right fusiform gyri

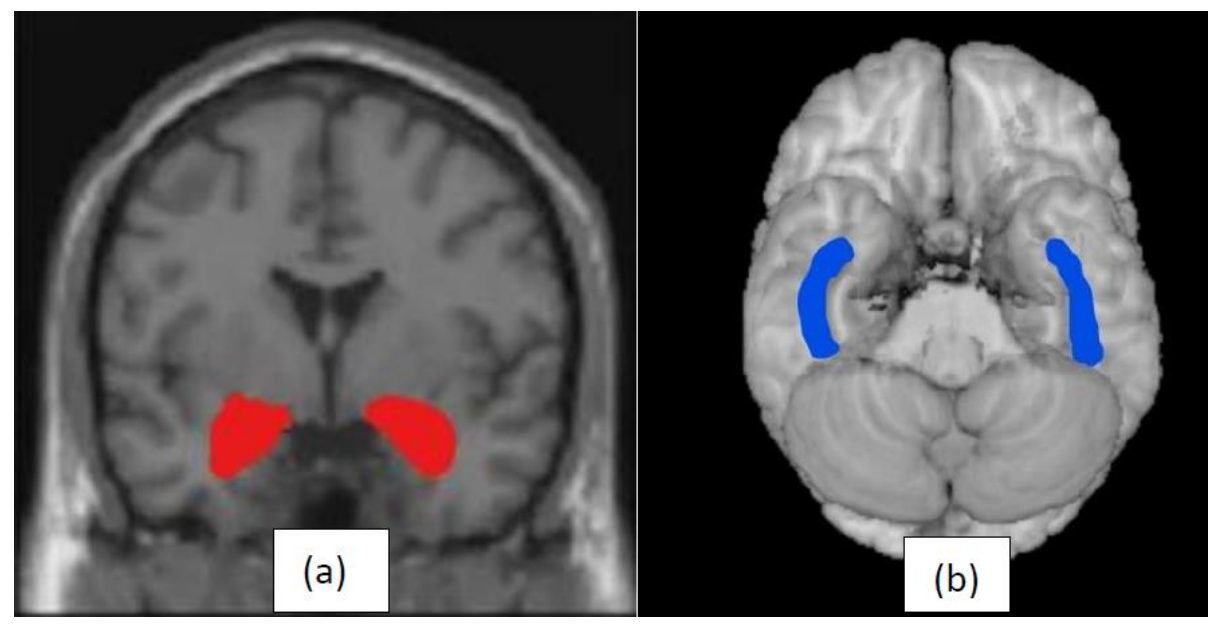

Statistical Analysis

A series of repeated measures multivariate analysis of variance (MANOVA) tests (SPSS ver 24, IBM Corp.) examining: (1) activation in the regions of interest (2(stress, no 
stress)*4 (ROIs)), (2) average functional connectivity across ROIs (2-way MANOVA, (stress, no stress)*overall functional connectivity) and (3) functional connectivity strength between individual ROI pairs (2 (stress, no stress)* 6 (ROI pairs)). The analyses were repeated with age and gender as between subject factors in order to assess the interaction effects of these factors.

\section{RESULTS}

\section{Demographics}

Functional magnetic resonance imaging data from 30 participants was analyzed (5 participants were dropped from the study because they did not attend the second session and data from 10 participants was not included in further analysis due to excessive motion within the scanner, i.e., relative motion more than $2 \mathrm{~mm}$ along any of the linear $(\mathrm{x}, \mathrm{y}, \mathrm{z})$ or rotational axes (roll, pitch, yaw)). Of the 30 participants included in the final analysis, there were 9 male participants and 13 participants had the $S$-allele. (Table 4.1).

Table 4.1 Demographics of participants 


\begin{tabular}{|c|c|c|c|c|c|c|c|c|c|}
\hline & $\begin{array}{c}\text { Total } \\
(\mathrm{n}=\mathbf{3 0})\end{array}$ & Male & Female & $\mathrm{X}^{2}$ & $\mathrm{p}$ & $\begin{array}{l}\text { S-allele } \\
(\mathrm{n}=13)\end{array}$ & $\begin{array}{c}\begin{array}{c}\text { L-allele } \\
(\mathrm{n}=17)\end{array}\end{array}$ & $\mathrm{X}^{2}$ & $\mathrm{P}$ \\
\hline $\begin{array}{c}\text { AGE } \\
\text { (YEARS) }\end{array}$ & $\begin{array}{l}19.8 \\
(1.7)\end{array}$ & $\begin{array}{l}20.0 \\
(2.2)\end{array}$ & $\begin{array}{l}19.8 \\
(1.5)\end{array}$ & 8.715 & 0.121 & $\begin{array}{l}19.5 \\
(1.1)\end{array}$ & $\begin{array}{l}20.0 \\
(2.0)\end{array}$ & 6.729 & 0.242 \\
\hline $\begin{array}{l}\text { GENDER } \\
(\mathbf{M} / \mathbf{F})\end{array}$ & $9 / 21$ & - & - & - & - & $4 / 9$ & $5 / 12$ & - & - \\
\hline \multicolumn{10}{|c|}{$\begin{array}{l}\text { GROUP COMPARISONS WERE PERFORMED USING CHI-SQUARE TEST. ALL VALUES ARE } \\
\text { SHOWN AS MEAN(SD). }\end{array}$} \\
\hline
\end{tabular}

STAI scores pre and post the imaging session were not significantly different as reported earlier (Chapter 2, Results: Demographics).

ROI activation

Figure 4.3 displays the group activation maps for participants $(n=30)$ while viewing emotional faces under (a) stress and (b) no stress.

Figure 4.3 Group activation maps. Group activation maps for participants $(n=30)$ viewing emotional faces under (a) stress and (b) no stress.

(a) ${ }^{\mathrm{T}}$ 


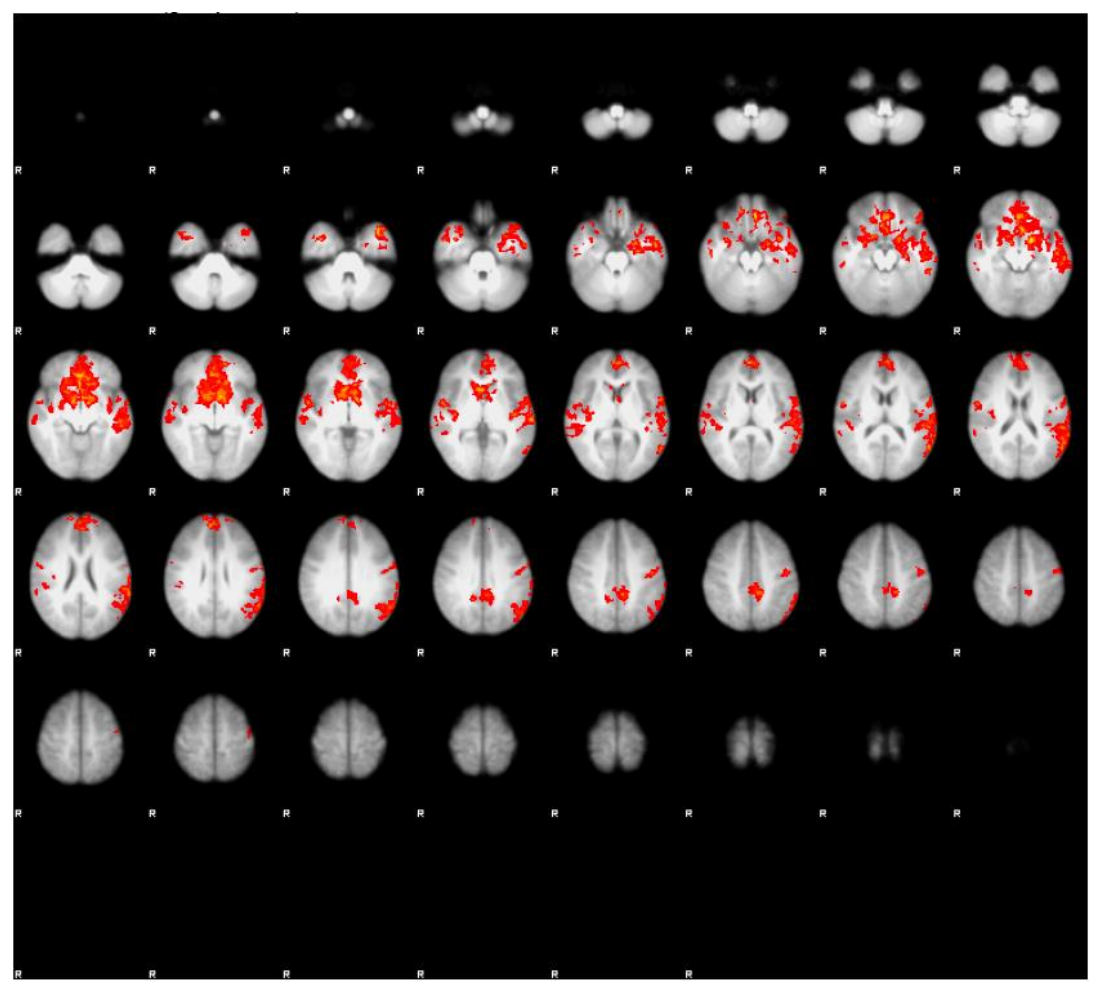

(b) Thresholded activation images

2.3

5.1

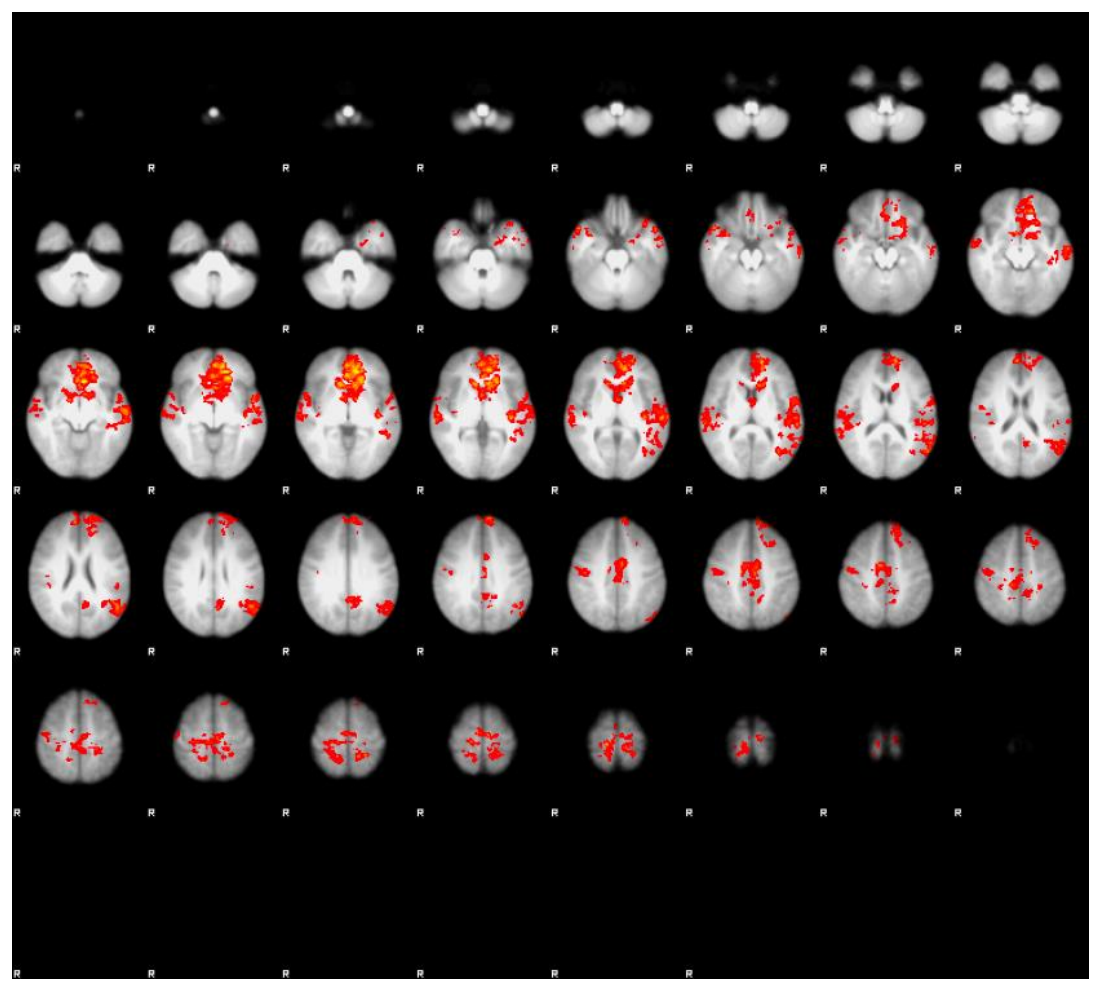


While a main effect of stress on overall activation across the regions was not observed $(F(1,26)=2.298, p=0.142)$, a stress $\mathrm{x}$ gender $\mathrm{x}$ allele interaction effect was observed on activation in the left amygdala $(F(1,26)=4.256, p=0.04)$ (Figure 4.4). Post hoc t-tests revealed that L-allele males had significantly greater mean activation in the left amygdala under stress compared to L-allele females $(\mathrm{t}=2.136, p=0.05)$. No other significant differences were observed between the individual groups under conditions of stress vs. no stress.

Figure 4.4 Differences in mean activation in left amygdala while viewing emotional faces under stress and no stress. A significant stress $x$ gender $x$ allele interaction effect was observed on activation in the left amygdala $(F(1,26)=4.256, p=0.04)$. Post hoc t-tests showed that L-allele males had increased activation in the region while viewing emotional faces than L-allele females $(\mathrm{t}=2.136, p=0.05)$. 


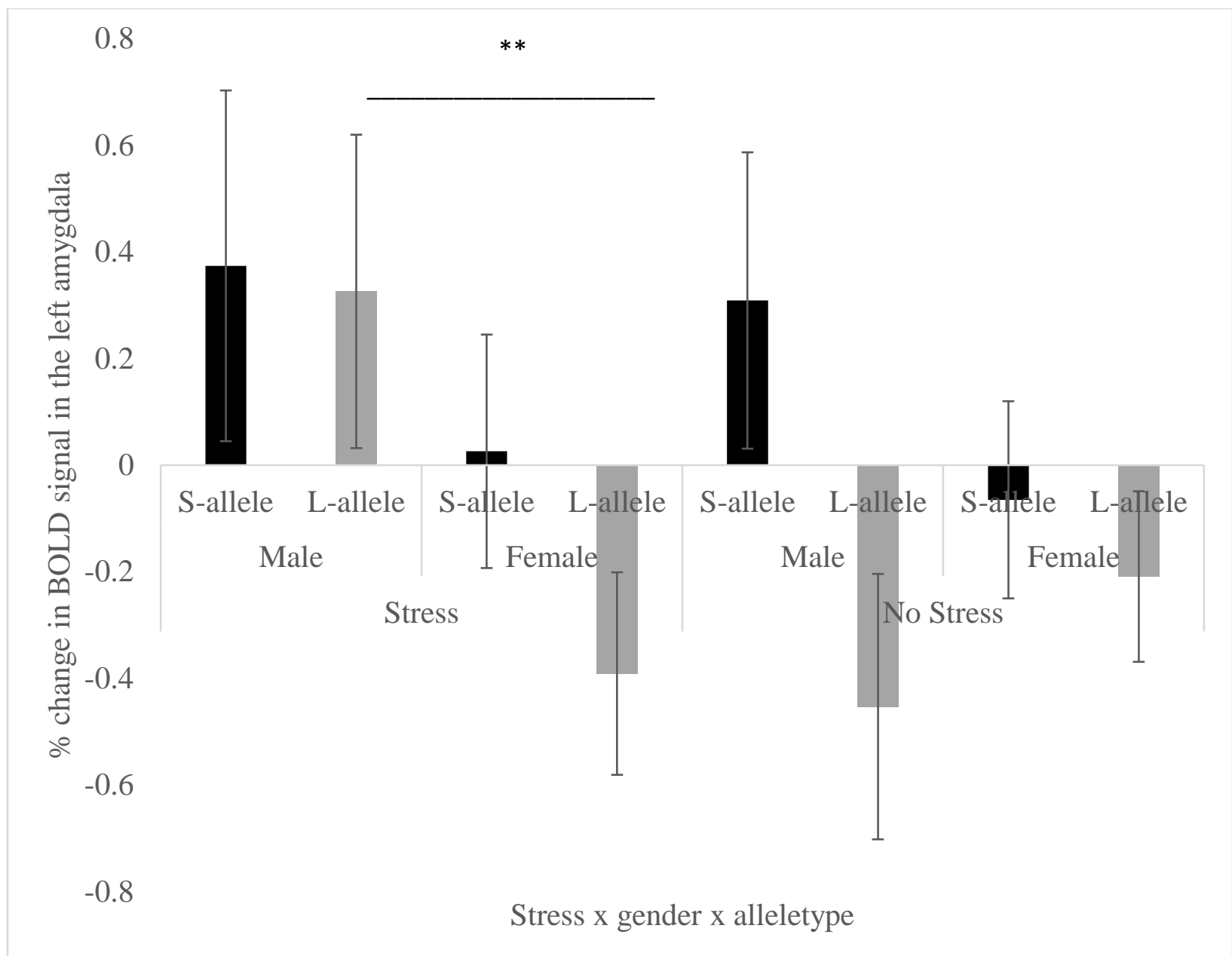

Stress $(F(1,26)=0.197, p=0.661)$, stress $\mathrm{x}$ gender $(F(1,26)=1.279, p=0.268)$ or stress $\mathrm{x}$ allele $(F(1,26)=0.574, p=0.456)$ did not produce significant effects on overall functional connectivity between the right amygdala, left amygdala, right fusiform gyrus and the left fusiform gyrus. Nevertheless, possible variations in functional connectivity between individual pairs was still of interest, specifically those of the left amygdala due to the observed gender and allele type specific changes in mean activation noted in the area. However, the gender and allele type specific difference in mean activation observed in the left amygdala did not result in a significant change in the functional connectivity between the left amygdala and the right amygdala, right fusiform or left fusiform gyri. 
We did not observe any significant differences in functional connectivity between the amygdala and the fusiform gyrus when viewing emotional faces under stress and no stress.

\section{DISCUSSION}

This study aimed to explore the effect of stress on functional connectivity between the amygdala and fusiform gyrus while viewing emotional faces.

Activation in the left amygdala was found to be significantly different between L-allele males and L-allele females under stress with L-allele males displaying much greater amplitudes of activation than L-allele females. Activation in the left amygdala during emotional processing observed here has been noted previously too (Baas, Aleman, \& Kahn, 2004). One other study has reported on the effects of stress on L-allele individuals, in an all-male cohort, where they observed increased stress sensitivity in L-allele subjects (Ming et al., 2015), consistent with what we have observed here, even though the difference in activation under stress and no stress for L-allele males was not found to be statistically significant in our study. Replication of this study in a larger sample will be needed to further analyze the influence of L-allele on the amygdala response to stress in females while viewing emotional faces.

Contrary to our hypothesis, we did not observe an upregulation in connectivity between the regions under stress. It is possible that the lack of influence of stress on amygdala- 
fusiform functional connectivity observed here might have to do with temporally variable effects of stress. Quaedflieg et al., 2015 reported on how resting state amygdala functional connectivity is altered in a time dependent manner following exposure to acute stress. Using an EEG paradigm, Shackman, Maxwell, McMenamin, Greischar and Davidson found that stress caused an initial amplification of N1 signal (184-236 ms) followed by an attenuation of the P3 signal (316-488 ms) during a visual discrimination task with potential threat of electric shock. The authors interpreted this finding as initial hypervigilance towards the stress followed by a disruption of cognitive processing. Since fMRI data while viewing emotional faces was collected after the stress inducing block and the faces task appeared for a longer duration (48s) than the stress block (30s) (a total duration of $78 \mathrm{~s}$ since the initiation of stress), it is possible that the initial potentiating effects of stress on the amygdala-fusiform connection might have shifted towards attenuating effects on visual attention with time, resulting in no change overall across the whole task block. Further supporting this argument, previous studies reporting on stress related enhancement of activation within the fusiform gyrus and/or the amygdala were designed to use shorter durations of stimulus presentation e.g., $133 \mathrm{msec}$ for each of 10 different faces presented (Hein J F van Marle et al., 2009) and 20 faces presented at 1.5 msec per face for a total of $30 \mathrm{msec}$ (Mather, Lighthall, Nga, \& Gorlick, 2010) and presentation of distinct faces every $0.5 \mathrm{~s}$ for a total duration of $25 \mathrm{~s}$ (Henckens, van Wingen, Joels, \& Fernandez, 2010). Future research will need to investigate the specific temporal effects of stress on functional connectivity between the amygdala and the fusiform gyrus in order to draw more definitive conclusions. 
While the preliminary results indicate that stress does not have a role in altering the functional connectivity between the amygdala and the fusiform gyrus, the cohort of participants examined here were all healthy with no previous or current medical or psychiatric history. It would be interesting to explore how stress might affect the functional connectivity between the amygdala and fusiform gyrus in individuals with anxiety, posttraumatic stress disorder and depression. With these explorations, newer insights into the neuropathophysiological mechanisms underlying these conditions could be developed. Combined with the knowledge of potential ways to manipulate these systems, such as regulation of stress response through the noradrenergic system that has direct effects on the amygdala (Onur et al., 2009; Van Stegeren et al., 2005), this opens newer avenues to develop treatments addressing some of the core features of these disorders.

\section{LIMITATIONS}

The results of this studies must be interpreted with caution considering that there are some limitations. The sample size, especially number of males in the S-allele group, was somewhat small. Future studies will want to replicate the methods employed here in a larger sample to draw more definitive conclusions. While the MIST paradigm is known to induce stress with consistent cortisol responses (Dedovic, Rexroth, et al., 2009), for this study, we did not have reliable cortisol levels available for the participants.

\section{SUMMARY}


The current study explored whether stress upregulated the functional connectivity between the amygdala and the fusiform gyrus while viewing emotional faces. The additional influence of gender and genotype were also explored. Preliminary results indicate that stress does not influence the functional connectivity strength between the amygdala and fusiform gyrus. Stress did increase activation in the left amygdala in a gender and genotype specific manner. Future studies will need to explore the effects of stress on functional connectivity between the amygdala and fusiform gyrus during facial emotion recognition in clinical populations. This will subsequently help develop mechanisms to better treat conditions ranging from phobias to depression, anxiety and post-traumatic stress disorder. 


\section{CHAPTER FIVE:}

\section{CONCLUSIONS AND FUTURE DIRECTIONS}

Stress is known to negatively affect physiological and psychological processes in humans and has been implicated in a wide array of disorders, ranging from cardiovascular, immunological to neuropsychiatric such as anxiety, depression and post-traumatic stress disorder (Cohen et al., 2007; Dimsdale, 2008).

Since the brain is the obligate primary organ that perceives and responds to stress, considerable research has gone into understanding the neural underpinnings of stress pathology. Studies have reported on the changes in structural volume (Arnsten, 2009; McEwen et al., 2016) and mean activation in specific regions of the brain following stress exposure (Henckens et al., 2009; Oei et al., 2012; Ossewaarde et al., 2011; Shaozheng Qin et al., 2009; Hein J F van Marle et al., 2009). Resting state functional connectivity changes following stress exposure have also been reported (Golkar et al., 2014;

Quaedflieg et al., 2015; Soares et al., 2013; Vaisvaser et al., 2013; H. J F van Marle et al., 2010; Veer et al., 2011, 2012). In general, stress is associated with hypervigilance and narrowed, focused attention. As a result, conditions of stress can cause impairment in performance on tasks requiring cognitive flexibility, i.e., being able to sift through various presented solutions and form associations that are not readily available in order to arrive at an appropriate solution. The first two studies presented here looked at the effect of stress on functional connectivity during tasks requiring cognitive flexibility. 
In the first study, healthy participants underwent functional magnetic resonance imaging while performing letters and categories fluency tasks immediately following exposure to a stressor block (MIST) on one day and a no stress control block on another day. Though there were no significant differences in the number of words generated in either of the tasks under stress and no stress, we did find specific differences in neural activation and functional connectivity associated with verbal fluency task performance under stress and no stress. Results from the study reveal that during letter fluency task, stress appears to place additional load on long distance functional connections, such as between the left inferior frontal gyrus and the left fusiform gyrus during verbal fluency tasks.

Additionally, males were found to strengthen a shorter distance functional connection between the left inferior frontal gyrus and the left parietal lobe under stress, indicating a possible compensatory effect for the reduced activation observed in the parietal lobe following exposure to stress. This then begins to reveal specific neural underpinnings for differential stress susceptibility among the genders. During category fluency tasks, stress was found to impair connectivity between the left inferior frontal gyrus and the left middle temporal gyrus in a gender and genotype specific manner. These observations raise important questions on how gender and genotype might affect neuroimaging markers of and neural mechanisms involved in the negative effects of stress in situations that require greater levels of verbal fluency such as during public speaking (Faigel, 1987, 1991; Laverdure \& Boulenger, 1991). It also opens up newer avenues towards understanding the mechanism of action of therapeutic interventions, not only in healthy individuals but also in anxiety prone clinical populations. 
The second study examined the neural correlates of the effects of stress on cognitive flexibility. As with the first study, healthy participants were exposed to the Montreal Imaging Stress Test (MIST) to induce stress (and on a separate day, a no stress control task) immediately followed by task blocks consisting of the Compound Remote Associates task. The compound remote associates task requires searching through a wide, distributed network in order to come up with a single solution that forms a compound word with each of the three presented prompts.

Since stress is associated with heightened arousal and narrowed focus (Martindale \& Greenough, 1973), the ability to access such a distributed network in order to solve the CRA tasks is compromised during stress (J. K. Alexander et al., 2007). Therefore, stress would have direct effects on the neural correlates of such verbal problem-solving tasks requiring cognitive flexibility. While the results of the study do not show differences in number of problems solved correctly under stress and no stress, we did observe a trend, driven by nonsignificant effects in opposite directions across genotypes, for genotype specific effects on solution latency for correct solutions under stress and no stress. Results from the study reveal that the effect of stress on the neural correlates of verbal problem solving is influenced by gender and genotype with stress significantly decreasing the strength of functional connectivity in regions involved in frontal attentional control in S-allele males compared to S-allele females and L-allele males. Frontal control exerted during cognitive flexibility tasks that require searching through a wide conceptual space help with staying on task and a decrease in functional connectivity strength in this connection could begin to explain the individual differences in the 
mechanisms associated with the effects of stress on cognition. Once again, while looking at therapeutic interventions to optimize flexible thinking in unfavorable circumstances, the specific influences of gender and genotype need to be taken into consideration due to the differences in neural correlates associated with these factors.

The third study explored how stress affected functional connectivity between the amygdala and fusiform gyrus while viewing emotional faces. Previous studies have reported on enhanced amygdala activation while viewing emotional faces under stress (Cousijn et al., 2010; Hein J F van Marle et al., 2009; Whalen et al., 2001). This study explored if the enhanced amygdala activation translates to upregulated functional connectivity with the fusiform gyrus, and subsequently, increased attention to negative stimuli. While gender and genotype related differences in activation in the left amygdala were noted, functional connectivity upregulation between the amygdala and fusiform gyrus was not observed. A potential explanation for this observation could be the temporal dynamics of effect of stress on amygdala functional connectivity, as has been reported in previous studies. For example, resting state functional connectivity of the amygdala has been reported to be altered by exposure to acute stress in a time dependent manner. Immediate changes in connectivity following to exposure to stress were observed between amygdala and ventrolateral prefrontal cortex, ventral posterior cingulate cortex, cuneus, parahippocampal gyrus and culmen. However, 30 minutes following exposure to the acute stressors, changes in functional connectivity were reported between the amygdala and the dorsolateral prefrontal cortex, anterior cingulate cortex, hippocampal complex, cuneus and presupplementary motor area (Quaedflieg et 
al., 2015). Future studies monitoring amygdala functional connectivity changes over shorter durations of time following stress exposure will be required to address this issue.

While the studies presented here looked at healthy participants, these investigations have similar if not greater significance in clinical populations. Examining the neural correlates of stress during verbal fluency in patient populations with anxiety and depression may help elucidate the neuropathophysiology underlying such conditions and serve as a potential marker for clinical populations. Furthermore, heightened amygdala response to emotional faces is a classic characteristic of a number of neuropsychiatric conditions such as post-traumatic stress disorder (Bryant et al., 2008; Rauch et al., 2000) and generalized anxiety disorder (Monk \& Telzer, 2008). Understanding what drives this hyperresponsiveness (factors such as gender and genotype) as well as the subsequent effects of this hyper reactivity on other brain regions could help clarify the neuropathology behind the development of stress associated disorders such as phobias, anxiety and depression. With the availability of pharmacological interventions that are known to affect amygdala activity (Onur et al., 2009; Van Stegeren et al., 2005), future research will have to look into sorting out the array of potential markers of stress susceptibility. As mentioned previously, though pharmacological interventions such as the use of the beta-adrenergic antagonist, propranolol, are known to rescue the negative effects of stress on cognitive performance, they are found to be effective only in specific conditions such as when the problems presented are at a greater level of difficulty (Campbell et al., 2008) or when there is increased noradrenergic tone from cocaine withdrawal (Kelley et al., 2007). 
Therefore, a more comprehensive study of the specific effects of stress on the neural correlates of cognition is warranted.

Future studies will need to replicate the findings here in bigger sample sizes in order to draw more definitive conclusions and validate the utility of functional connectivity as a biomarker for increased stress susceptibility in certain settings. While results from the studies presented here begin to elucidate the influence of gender and serotonin transporter polymorphisms on neural correlates of stress susceptibility during cognitive tasks, in order to gain a more comprehensive understanding of stress susceptibility, and to further validate functional connectivity as a biomarker of stress susceptibility, a number of other factors will need to be considered. In an animal model, Inglis \& Moghaddam, 1999 showed that acute stress caused significantly greater release of dopamine in the amygdala than other areas of the brain with dopaminergic innervation. Additionally, dopamine, its receptor systems and associated genes are also known to have specific influences on cognitive flexibility tasks (Beversdorf, 2018). Therefore, future studies should consider modulation of dopaminergic systems in order to evaluate its effect on cognitive abilities under stress.

The influence of early life stress as well as prenatal stress on stress susceptibility is an area of extensive research. Early life adversity has been associated with long term changes in stress susceptibility and reactivity, leading to increased risk for psychiatric conditions such as post-traumatic stress disorder and major depression (Pechtel \& Pizzagalli, 2011). J. C. Pruessner et al., 2010 reported that dopamine release in 
mesolimbic regions in response to stress was characteristic of individuals with increased stress sensitivity arising from possible self-reported lower maternal care during early life. Early life stress has been reported to produce robust alterations in functional connectivity and structural volume within the brain (Admon et al., 2013; Fan et al., 2014, 2015) further elucidating the potential use of neuroimaging as a biomarker for stress susceptibility. Additionally, influence of early life stress on neural responses has also been studied as a potential marker for prediction of treatment response with oxytocin (Grimm et al., 2014). This study reported that oxytocin, meant to relieve the negative effects associated with stress, caused an opposite response in patients who had experienced early life stress, such as childhood maltreatment by causing extensive activation of the limbic system. Another factor to consider would be in utero exposure to stress which can also have a major impact of subsequent development of stress related and other neuropsychiatric disorders and therefore, cognitive ability (Bock, Wainstock, Braun, \& Segal, 2015; Fine, Zhang, \& Stevens, 2014). The influence of early life stress on stress sensitivity and susceptibility in later life is therefore, an important factor to consider when studying potential markers for stress susceptibility.

In the studies presented here, information on the phase of menstrual cycle for females was not available. Reliable data on the use of birth control medication was also unavailable. Both these factors are known to impact stress response, cognition and emotion in females (Goldstein, Jerram, Abbs, Whitfield-gabrieli, \& Makris, 2010; ter Horst, de Kloet, Schachinger, \& Oitzl, 2012; Toffoletto, Lanzenberger, Gingnell, Sundström-poromaa, \& Comasco, 2014; N C Vamvakopoulos \& Chrousos, 1993; 
Nicholas C Vamvakopoulos \& Chrousos, 1994; Weis, Hausmann, Stoffers, \& Sturm, 2011) and are therefore, important considerations for future studies looking to examine neural correlates of stress effects on cognition.

There are some preliminary studies examining the effects of stress on neurovascular coupling within the brain (Elbau et al., 2018). A more extensive examination of these possible variations in neurovascular coupling due to stress is warranted in order to further validate the use of neuroimaging as a biomarker for stress susceptibility.

It is also worth noting that apart from the serotonin transporter gene considered here, a number of other genes are involved in regulating stress related neural activity such as the COMT $\mathrm{Val}^{158}$ Met genotype, involved in breaking down of catecholamines such as dopamine and norepinephrine, known to influence resting state connectivity and working memory (Hernaus et al., 2013; Shaozheng Qin et al., 2012) and polymorphisms of the $A D R A 2 B$ gene encoding the alpha2B-adrenergic receptor reported to differentially modulate activity in the amygdala and hippocampus during emotional memory retrieval under stress (Li, Weerda, Milde, Wolf, \& Thiel, 2015). Future studies will need to incorporate more comprehensive genomic profiles to understand how the presence of these genes might affect individual susceptibility to stress and cognitive functioning.

Information about the influence of these potential factors on the neural correlates of stress susceptibility and sensitivity during cognitive functioning will help in the creations of neuropharmacogenomic profiles of stress susceptibility for individuals. These in turn, 
will help identify and target the specific pathways affected in each individual, thereby, paving way towards developing more targeted therapeutic interventions in the treatment of stress related cognitive deficits and disorders. 


\section{REFERENCES}

Abraham, A. (2016). Gender and creativity: an overview of psychological and neuroscientific literature. Brain Imaging and Behavior, 10(2), 609-618. https://doi.org/10.1007/s11682-015-9410-8

Abraham, A., Thybusch, K., Pieritz, K., \& Hermann, C. (2014). Gender differences in creative thinking: Behavioral and fMRI findings. Brain Imaging and Behavior, 8(1), 3951. https://doi.org/10.1007/s11682-013-9241-4

Admon, R., Leykin, D., Lubin, G., Engert, V., Andrews, J., Pruessner, J., \& Hendler, T. (2013). Stress-induced reduction in hippocampal volume and connectivity with the ventromedial prefrontal cortex are related to maladaptive responses to stressful military service. Human Brain Mapping, 34(11), 2808-2816. https://doi.org/10.1002/hbm.22100

Aldwin, C.; Sutton, K.; Chiara, G. \& Avron, S. (1996). Age Differences in Stress, Coping, and Appraisal: Findings From the Normative Aging Study. Journal of Gerontology: PSYCHOLOGICAL SCIENCES, 5(4), 79-188. https://doi.org/10.1093/geronb/51B.4.P179

Alexander, J. K., Hillier, A., Smith, R. M., Tivarus, M. E., \& Beversdorf, D. Q. (2007). Beta-adrenergic Modulation of Cognitive Flexibility during Stress. Journal of Cognitive Neuroscience, 19(3), 468-478. https://doi.org/10.1162/jocn.2007.19.3.468

Alexander, N., Klucken, T., Koppe, G., Osinsky, R., Walter, B., Vaitl, D., ... Hennig, J. (2012). Interaction of the serotonin transporter-linked polymorphic region and environmental adversity: Increased amygdala-hypothalamus connectivity as a potential mechanism linking neural and endocrine hyperreactivity. Biological Psychiatry, 72(1), 49-56. https://doi.org/10.1016/j.biopsych.2012.01.030 
Andrews, J., Ali, N., \& Pruessner, J. C. (2013). Reflections on the interaction of psychogenic stress systems in humans: The stress coherence/compensation model. Psychoneuroendocrinology, 38(7), 947-961. https://doi.org/10.1016/j.psyneuen.2013.02.010

Andrews, J., \& Pruessner, J. C. (2013). The Combined Propranolol/TSST Paradigm - A New Method for Psychoneuroendocrinology. PLoS ONE, 8(2). https://doi.org/10.1371/journal.pone.0057567

Arnsten, A. F. T. (2009). Stress signalling pathways that impair prefrontal cortex structure and function. Nature Reviews Neuroscience, 10(6), 410-422. https://doi.org/10.1038/nrn2648

Baas, D., Aleman, A., \& Kahn, R. S. (2004). Lateralization of amygdala activation: A systematic review of functional neuroimaging studies. Brain Research Reviews, 45(2), 96-103. https://doi.org/10.1016/j.brainresrev.2004.02.004

Baron-Cohen, S., Ring, H. A., Bullmore, E. T., Wheelwright, S., Ashwin, C., \& Williams, S. C. R. (2000). The amygdala theory of autism. Neuroscience and Biobehavioral Reviews, 24(3), 355-364. https://doi.org/10.1016/S0149-7634(00)00011-7

Bartha, L., Brenneis, C., Schocke, M., Trinka, E., Koylu, B., Trieb, T., ... Benke, T. (2003). Medial temporal lobe activation during semantic language processing : fMRI findings in healthy left- and right-handers. Cognitive Brain Research, 17, 339-346.

Beaty, R. E., Benedek, M., Wilkins, R. W., Jauk, E., Fink, A., Silvia, P. J., ... Neubauer, A. C. (2014). Creativity and the default network: A functional connectivity analysis of the creative brain at rest. Neuropsychologia, 64, 92-98. 
https://doi.org/10.1016/j.neuropsychologia.2014.09.019

Bebbington, P. E. (1998). Sex and depression. Psychological Medicine, 28(1), 1-8. https://doi.org/10.1017/S0033291797006065

Bell, E. C., Willson, M. C., Wilman, A. H., Dave, S., \& Silverstone, P. H. (2006). Males and females differ in brain activation during cognitive tasks. NeuroImage, 30, 529-538. https://doi.org/10.1016/j.neuroimage.2005.09.049

Bengel, D., Murphy, D. L., Andrews, A. M., Wichems, C. H., Feltner, D., Heils, A., ... Lesch, K. P. (1998). Altered brain serotonin homeostasis and locomotor insensitivity to 3, 4-methylenedioxymethamphetamine ("Ecstasy") in serotonin transporter-deficient mice. Molecular Pharmacology, 53(4), 649-655. https://doi.org/10.1124/MOL.53.4.649

Beversdorf, D. Q. (2018). Neuropsychopharmacological regulation of performance on creativity-related tasks. Current Opinion in Behavioral Sciences, In press.

Beversdorf, D. Q., Carpenter, A. L., Alexander, J., Jenkins, N., Tilley, M. R., White, C., ... Gu, H. (2017). Exploratory Pilot Study of Influence of Serotonin Transporter Genotype on the Effect of Psychosocial Stress on Cognitive Performance. Cognitive and Behavioral Neurology, In press.

Beversdorf, D. Q., Carpenter, A. L., Miller, R. F., Cios, J. S., \& Hillier, A. (2008). Effect of propranolol on verbal problem solving in autism spectrum disorder. Neurocase, 14(4), 378-383. https://doi.org/10.1080/13554790802368661

Beversdorf, D. Q., Hughes, J. D., Steinberg, B. A., Lewis, L. D., \& Heilman, K. M. (1999). Noradrenergic modulation of cognitive flexibility in problem solving. In Journal of Cognitive Neuroscience (Vol. S, p. 60). 
Beversdorf, D. Q., Saklayen, S., Higgins, K. F., Bodner, K. E., Kanne, S. M., \& Christ, S. E. (2011). Effect of propranolol on word fluency in autism. Cognitive and Behavioral Neurology, 24(1), 11-17. https://doi.org/10.1097/WNN.0b013e318204d20e

Beversdorf, D. Q., Sharma, U. K., Phillips, N. N., Notestine, M. A., Slivka, A. P., Friedman, N. M., ... Hillier, A. (2007). Effect of Propranolol on Naming in Chronic Broca’s Aphasia with Anomia. Neurocase, 13, 256-259. https://doi.org/10.1080/13554790701595471

Beversdorf, D. Q., White, D. M., Chever, D. C., Hughes, J. D., \& Bornstein, R. A. (2002). Central beta-adrenergic modulation of cognitive flexibility. NeuroReport, 13(18), 2505-2507. https://doi.org/10.1097/01.wnr.0000048923.00321.a7

Bock, J., Wainstock, T., Braun, K., \& Segal, M. (2015). Stress In Utero: Prenatal Programming of Brain Plasticity and Cognition. Biological Psychiatry, 78(5), 315-326. https://doi.org/10.1016/j.biopsych.2015.02.036

Bowden, E. M., \& Jung-beeman, M. (2003). Aha! Insight experience correlates with solution activation in the right hemisphere. Psychonomic Bulletin \& Review, 10(3), 730737.

Bowden, E. M., \& Jung-Beeman, M. (2003). Normative data for 144 compound remote associate problems. Behavior Research Methods, Instruments, and Computers, 35(4), 634-639. https://doi.org/10.3758/BF03195543

Bremner, J. D., Krystal, J. H., Southwick, S. M., \& Charney, D. S. (2006). Noradrenergic mechanisms in stress and anxiety .2. Clinical studies. Synapse, 23(1), 39-51. https://doi.org/10.1002/(SICI)1098-2396(199605)23:1<39::AID-SYN5>3.0.CO;2-I 
Bromet, E., Andrade, L. H., Hwang, I., Sampson, N. A., Alonso, J., de Girolamo, G., ... Kessler, R. C. (2011). Cross-national epidemiology of DSM-IV major depressive episode. BMC Medicine, 9(1), 90. https://doi.org/10.1186/1741-7015-9-90

Bryant, R. A., Kemp, A. H., Felmingham, K. L., Liddell, B., Olivieri, G., Peduto, A., ... Williams, L. M. (2008). Enhanced amygdala and medial prefrontal activation during nonconscious processing of fear in posttraumatic stress disorder: An fMRI study. Human Brain Mapping, 29(5), 517-523. https://doi.org/10.1002/hbm.20415

Cai, D. J., Mednick, S. A., Harrison, E. M., Kanady, J. C., \& Mednick, S. C. (2009). REM, not incubation, improves creativity by priming associative networks. Proceedings of the National Academy of Sciences, 106(25), 10130-10134. https://doi.org/10.1073/pnas.0900271106

Campbell, H. L., Tivarus, M. E., Hillier, A., \& Beversdorf, D. Q. (2008). Increased task difficulty results in greater impact of noradrenergic modulation of cognitive flexibility. Pharmacology Biochemistry and Behavior, 88, 222-229. https://doi.org/10.1016/j.pbb.2007.08.003

Caspi, A., Sugden, K., Moffitt, T., Taylor, A., Craig, I., \& Harrington, H. (2003). Influence of life stress on depresson: Moderation by a polymorphism in the 5-HTT gene. Science, 301(July), 386-389.

Caspi, A., Hariri, A. R., Holmes, A., Uher, R., \& Moffitt, T. (2010). Genetic Sensitivity to the Environment: The Case of the Serotonin Transporter Gene and Its Implications for Studying Complex Diseases and Traits. American Journal of Psychiatry, 167, 509-527.

Chamberlain, S. R., \& Robbins, T. W. (2013). Noradrenergic modulation of cognition : 
Therapeutic implications. Journal of Psychopharmacology, 27(8), 694-718. https://doi.org/10.1177/0269881113480988

Chermahini, S. A., \& Hommel, B. (2010). The (b)link between creativity and dopamine: Spontaneous eye blink rates predict and dissociate divergent and convergent thinking. Cognition, 115(3), 458-465. https://doi.org/10.1016/j.cognition.2010.03.007

Chrousos, G. P. (2009). Stress and disorders of the stress system. Nat Rev Endocrinol, 5, 374-381. https://doi.org/10.1038/nrendo.2009.106

Cohen, S., Janicki-deverts, D., \& Miller, G. E. (2007). Psychological Stress and Disease. JAMA, 298(14), 1685-1687.

Costafreda, S. G., Fu, C. H. Y., Lee, L., Everitt, B., Brammer, M. J., \& David, A. S. (2006). A Systematic Review and Quantitative Appraisal of fMRI Studies of Verbal Fluency : Role of the Left Inferior Frontal Gyrus. Human Brain Mapping, 27, 799-810. https://doi.org/10.1002/hbm.20221

Cousijn, H., Rijpkema, M., Qin, S., Marle, H. J. F. Van, Franke, B., Hermans, E. J., ... Fernández, G. (2010). Acute stress modulates genotype effects on amygdala processing in humans. Proceedings of the National Academy of Sciences, 107(21), 9867-9872. https://doi.org/10.1073/pnas.1003514107

Dannlowski, U., Konrad, C., Kugel, H., Zwitserlood, P., Domschke, K., Schöning, S., ... Suslow, T. (2010). Emotion specific modulation of automatic amygdala responses by 5HTTLPR genotype. NeuroImage, 53(3), 893-898. https://doi.org/10.1016/j.neuroimage.2009.11.073

Dedovic, K., Aguiar, C. D., \& Pruessner, J. C. (2009). What Stress Does to Your Brain : 
A Review of Neuroimaging Studies. La Revue Canadienne de Psychiatrie, 54(1), 6-15.

Dedovic, K., Renwick, R., Mahani, N. K., Engert, V., Lupien, S. J., \& Pruessner, J. C. (2005). The Montreal Imaging Stress Task : using functional imaging to investigate the effects of perceiving and processing psychosocial stress in the human brain. Journal of Psychiatry and Neuroscience, 30(5), 319-325.

Dedovic, K., Rexroth, M., Wolff, E., Duchesne, A., Scherling, C., Beaudry, T., ... Pruessner, J. C. (2009). Neural correlates of processing stressful information: An eventrelated fMRI study. Brain Research, 1293, 49-60.

https://doi.org/10.1016/j.brainres.2009.06.044

Devlin, J. T., Jamison, H. L., Gonnerman, L. M., \& Matthews, P. M. (2006). The role of the posterior fusiform gyrus in reading. J Cogn Neurosci, 18(6), 911-922. https://doi.org/10.1162/jocn.2006.18.6.911.

Dimsdale, J. E. (2008). Psychological Stress and Cardiovascular Disease. Journal of the American College of Cardiology, 51(13), 1237-1246.

https://doi.org/10.1016/j.jacc.2007.12.024

Drabant, E. M., Ramel, W., Edge, M. D., Hyde, L. W., Kuo, J. R., Goldin, P. R., ... Gross, J. J. (2012). Neural Mechanisms Underlying 5-HTTLPR-Related Sensitivity to Acute Stress. American Journal of Psychiatry, 169, 397-405.

Dukal, H., Frank, J., Lang, M., Treutlein, J., Gilles, M., Wolf, I. A. C., ... Witt, S. H. (2015). New-born females show higher stress- and genotype-independent methylation of SLC6A4 than males. Borderline Personality Disorder and Emotion Dysregulation, 2(8), 4-11. https://doi.org/10.1186/s40479-015-0029-6 
Duman, E. A., \& Canli, T. (2015). Influence of life stress , 5-HTTLPR genotype , and SLC6A4 methylation on gene expression and stress response in healthy Caucasian males. Biology of Mood and Anxiety Disorders, 5(2), 1-14. https://doi.org/10.1186/s13587-0150017-X

Dziobek, I., Bahnemann, M., Convit, A., \& Heekeren, H. R. (2010). The Role of the Fusiform - Amygdala System in the Pathophysiology of Autism. Arch Gen Psychiatry, 67(4), 397-405. https://doi.org/10.1001/archgenpsychiatry.2010.31

Easterbrook, J. A. (1959). The effect of emotion on cue utilization and the organization of behavior. Psychological Review, 66(3), 183-201. https://doi.org/10.1037/h0047707

Elbau, I. G., Brücklmeier, B., Uhr, M., Arloth, J., Czamara, D., Spoormaker, V. I., ... Samann, P. G. (2018). The brain's hemodynamic response function rapidly changes under acute psychosocial stress in association with genetic and endocrine stress response markers. PNAS, 115(43), E10206-E10215. https://doi.org/10.1073/pnas.1804340115

Epel, E. S., Blackburn, E. H., Lin, J., Dhabhar, F. S., Adler, N. E., Morrow, J. D., \& Cawthon, R. M. (2004). Accelerated telomere shortening in response to life stress. PNAS, 101(49), 17312-17315.

Faigel, H. C. (1987). The effect of beta blockade on scholastic aptitude test scores in adolescents. Journal of Adolescent Health Care, 8(3), 304.

Faigel, H. C. (1991). The effect of beta blockade on stress-induced cognitive dysfunction in adolescents. Clinical Pediatrics, 30(7), 441-445.

Fan, Y., Herrera-Melendez, A. L., Pestke, K., Feeser, M., Aust, S., Otte, C., ... Grimm, S. (2014). Early life stress modulates amygdala-prefrontal functional connectivity: 
Implications for oxytocin effects. Human Brain Mapping, 35(10), 5328-5339.

https://doi.org/10.1002/hbm.22553

Fan, Y., Pestke, K., Feeser, M., Aust, S., Pruessner, J. C., Böker, H., ... Grimm, S.

(2015). Amygdala-hippocampal connectivity changes during acute psychosocial stress: Joint effect of early life stress and oxytocin. Neuropsychopharmacology, 40(12), 27362744. https://doi.org/10.1038/npp.2015.123

Feinberg, T. E., Rifkin, A., Schaffer, C., \& Walker, E. (1986). Facial Discrimination and Emotional Recognition in Schizophrenia and Affective Disorders. Archives of General Psychiatry, 43(3), 276-279. https://doi.org/10.1001/archpsyc.1986.01800030094010

Fiez, J. (1997). Phonology, semantics and the tole of the left inferior prefrontal cortex. Human Brain Mapping, 5(March), 79-83.

Fine, R., Zhang, J., \& Stevens, H. E. (2014). Prenatal stress and inhibitory neuron systems: implications for neuropsychiatric disorders. Molecular Psychiatry, 19(6), 641651. https://doi.org/10.1038/mp.2014.35.

Flinker, A., Korzeniewska, A., Shestyuk, A. Y., Franaszczuk, P. J., \& Dronkers, N. F. (2015). Redefining the role of Broca' s area in speech. PNAS, 112(9), 2871-2875. https://doi.org/10.1073/pnas.1414491112

Franklin, T. B., Saab, B. J., \& Mansuy, I. M. (2012). Neural Mechanisms of Stress Resilience and Vulnerability. Neuron, 75(5), 747-761. https://doi.org/10.1016/j.neuron.2012.08.016

Gauthier, C. T., Duyme, M., Zanca, M., \& Capron, C. (2009). Sex and performance level effects on brain activation during a verbal fluency task : A functional magnetic resonance 
imaging study. CORTEX, 45, 164-176. https://doi.org/10.1016/j.cortex.2007.09.006

Gold, R., Faust, M., \& Ben-Artzi, E. (2012). Metaphors and verbal creativity: The role of the right hemisphere. Laterality, 17(5), 602-614.

https://doi.org/10.1080/1357650X.2011.599936

Goldstein, J. M., Jerram, M., Abbs, B., Whitfield-gabrieli, S., \& Makris, N. (2010). Sex Differences in Stress Response Circuitry Activation Dependent on Female Hormonal Cycle. The Journal of Neuroscience, 30(2), 431-438. https://doi.org/10.1523/JNEUROSCI.3021-09.2010

Golkar, A., Johansson, E., Kasahara, M., Osika, W., Perski, A., \& Savic, I. (2014). The influence of work-related chronic stress on the regulation of emotion and on functional connectivity in the brain. PLoS ONE, 9(9). https://doi.org/10.1371/journal.pone.0104550

Green, A. E., Kraemer, D. J. M., Fugelsang, J. A., Gray, J. R., \& Dunbar, K. N. (2012). Neural correlates of creativity in analogical reasoning. Journal of Experimental Psychology: Learning Memory and Cognition, 38(2), 264-272. https://doi.org/10.1037/a0025764

Green, A. E., Spiegel, K. A., Giangrande, E. J., Weinberger, A. B., Gallagher, N. M., \& Turkeltaub, P. E. (2017). Thinking cap plus thinking zap: Tdcs of frontopolar cortex improves creative analogical reasoning and facilitates conscious augmentation of state creativity in verb generation. Cerebral Cortex, 27(4), 2628-2639. https://doi.org/10.1093/cercor/bhw080

Grimm, S., Pestke, K., Feeser, M., Aust, S., Weigand, A., Wang, J., ... Bajbouj, M. (2014). Early life stress modulates oxytocin effects on limbic system during acute psychosocial stress. Social Cognitive and Affective Neuroscience, 9(11), 1828-1835. 
https://doi.org/10.1093/scan/nsu020

Gur, R. E., McGrath, C., Chan, R. M., Schroeder, L., Turner, T., Turetsky, B. I., ... Gur, R. C. (2002). An fMRI study of facial emotion processing in patients with schizophrenia. The American Journal of Psychiatry, 159(12), 1992-1999.

https://doi.org/10.1176/appi.ajp.159.12.1992

Gyawali, S., Subaran, R., Weissman, M. M., Hershkowitz, D., McKenna, M. C., Talari, A., ... Glatt, C. E. (2010). Association of a polyadenylation polymorphism in the serotonin transporter and panic disorder. Biol Psychiatry, 67(4), 331-338. https://doi.org/10.1016/j.biopsych.2009.10.015.Association

Hadjikhani, N., Joseph, R. M., Snyder, J., Chabris, C. F., Clark, J., Steele, S., ... TagerFlusberg, H. (2004). Activation of the fusiform gyrus when individuals with autism spectrum disorder view faces. NeuroImage, 22(3), 1141-1150. https://doi.org/10.1016/j.neuroimage.2004.03.025

Hagoort, P. (2014). Nodes and networks in the neural architecture for language: Broca' s region and beyond. Current Opinion in Neurobiology, 28, 136-141. https://doi.org/10.1016/j.conb.2014.07.013

Hariri, A. R., Drabant, E. M., \& Weinberger, D. R. (2006). Imaging Genetics : Perspectives from Studies of Genetically Driven Variation in Serotonin Function and Corticolimbic Affective Processing. Biol Psychiatry, 59, 888-897. https://doi.org/10.1016/j.biopsych.2005.11.005

Hariri, A. R., \& Holmes, A. (2006). Genetics of emotional regulation: the role of the serotonin transporter in neural function. Trends in Cognitive Sciences, 10(4), 182-191. https://doi.org/10.1016/j.tics.2006.02.011 
Hariri, A. R., Mattay, V. S., Tessitore, A., Kolachana, B. S., Fera, F., Goldman, D., ... Weinberger, D. R. (2002). Serotonin Transporter Genetic Variation and the Response of the Human Amygdala. Science, 297, 400-404.

Hayashi, T., MIzuno-Matsumoto, Y., Okamoto, E., Kato, M., \& Murata, T. (2012). An fMRI study of brain processing related to stress states. World Automation Congress, 1-6.

Hecht, P. M., Hudson, M., Connors, S. L., Tilley, M. R., Liu, X., \& Beversdorf, D. Q. (2016). Maternal Serotonin Transporter Genotype Affects Risk for ASD With Exposure to Prenatal Stress. Autism Research, 9, 1151-1160. https://doi.org/10.1002/aur.1629

Hecht, P. M., Will, M. J., Schachtman, T. R., Welby, L. M., \& Beversdorf, D. Q. (2014). Beta-adrenergic antagonist effects on a novel cognitive flexibility task in rodents. Behavioural Brain Research, 260, 148-154. https://doi.org/10.1016/j.bbr.2013.11.041

Heilman, K. M., Nadeau, S. E., \& Beversdorf, D. O. (2003). Creative Innovation: Possible Brain Mechanisms. Neurocase, 9(5), 369-379. https://doi.org/10.1076/neur.9.5.369.16553

Heils, A., Teufel, A., Petri, S., Stober, G., Riederer, P., Bengel, D., \& Lesch, K. (1996). Allelic Variation of Human Serotonin Transporter Gene Expression. Journal of Neurochemistry, 66(6), 2621-2624.

Henckens, M. J. A. G., Hermans, E. J., Pu, Z., Joels, M., \& Fernandez, G. (2009). Stressed Memories: How Acute Stress Affects Memory Formation in Humans. Journal of Neuroscience, 29(32), 10111-10119. https://doi.org/10.1523/JNEUROSCI.1184-09.2009 
Henckens, M. J. A. G., van Wingen, G. A., Joels, M., \& Fernandez, G. (2010). TimeDependent Effects of Corticosteroids on Human Amygdala Processing. Journal of Neuroscience, 30(38), 12725-12732. https://doi.org/10.1523/JNEUROSCI.3112-10.2010

Hermans, E. J., Henckens, M. J. A. G., Joëls, M., \& Fernández, G. (2014). Dynamic adaptation of large-scale brain networks in response to acute stressors. Trends in Neurosciences, 37(6), 304-314. https://doi.org/10.1016/j.tins.2014.03.006

Hermans, E. J., Marle, H. J. F. Van, Ossewaarde, L., Henckens, M. J. A. G., Qin, S., Kesteren, M. T. R., ... Fernandez, G. (2011). Stress-related noradrenergic activity prompts large-scale neural network reconfiguration. Science, 334(NOVEMBER), 11511153. https://doi.org/10.1126/science.1209603

Hernaus, D., Collip, D., Lataster, J., Ceccarini, J., Kenis, G., Booij, L., .. MyinGermeys, I. (2013). COMT Val158Met Genotype Selectively Alters Prefrontal [18F]Fallypride Displacement and Subjective Feelings of Stress in Response to a Psychosocial Stress Challenge. PLoS ONE, 8(6). https://doi.org/10.1371/journal.pone.0065662

Herrington, J. D., Taylor, J. M., Grupe, D. W., Curby, K. M., \& Schultz, R. T. (2011). Bidirectional communication between amygdala and fusiform gyrus during facial recognition. NeuroImage, 56(4), 2348-2355. https://doi.org/10.1016/j.neuroimage.2011.03.072

Hillier, A., Alexander, J. K., \& Beversdorf, D. Q. (2006). The Effect of Auditory Stressors on Cognitive Flexibility. Neurocase, 12, 228-231. https://doi.org/10.1080/13554790600878887

Hirshorn, E. A., \& Thompson-schill, S. L. (2006). Role of the left inferior frontal gyrus in 
covert word retrieval : Neural correlates of switching during verbal fluency.

Neuropsychologia, 44, 2547-2557.

https://doi.org/10.1016/j.neuropsychologia.2006.03.035

Inglis, F. M., \& Moghaddam, B. (1999). Dopaminergic innervation of the amygdala is highly responsive to stress. Journal of Neurochemistry, 72(3), 1088-1094.

https://doi.org/10.1046/j.1471-4159.1999.0721088.x

Ishizuka, K., Hillier, A., \& Beversdorf, D. Q. (2007). Effect of the Cold Pressor Test on Memory and Cognitive Flexibility. Neurocase, 13, 154-157.

https://doi.org/10.1080/13554790701441403

Jenkinson, M., Bannister, P., Brady, M., \& Smith, S. (2002). Improved optimization for the robust and accurate linear registration and motion correction of brain images. NeuroImage, 17(2), 825-841. https://doi.org/10.1016/S1053-8119(02)91132-8

Jenkinson, M., \& Smith, S. (2001). A global optimisation method for robust affine registration of brain images. Medical Image Analysis, 5(2), 143-156. https://doi.org/10.1016/S1361-8415(01)00036-6

Jiang, W., Shang, S., \& Su, Y. (2015). Genetic influences on insight problem solving: The role of catechol-O-methyltransferase (COMT) gene polymorphisms. Frontiers in Psychology, 6(OCT), 1-8. https://doi.org/10.3389/fpsyg.2015.01569

Jonides, J., Schumacher, E. H., Smith, E. E., Koeppe, R. A., Awh, E., Reuter-Lorenz, P. A., ... Willis, C. R. (1998). The role of parietal cortex in verbal working memory. Journal of Neuroscience, 18(13), 5026-5034.

Jung-Beeman, M. (2005). Bilateral brain processes for comprehending natural language. 
Trends in Cognitive Sciences, 9(11), 512-518. https://doi.org/10.1016/j.tics.2005.09.009

Jung-Beeman, M., Bowden, E. M., Haberman, J., Frymiare, J. L., Arambel-Liu, S., Greenblatt, R., ... Kounios, J. (2004). Neural activity when people solve verbal problems with insight. PLoS Biology, 2(4), 500-510. https://doi.org/10.1371/journal.pbio.0020097

Jung, R. E. (2013). The structure of creative cognition in the human brain. Frontiers in Human Neuroscience, 7(July), 1-13. https://doi.org/10.3389/fnhum.2013.00330

Jung, R. E., Grazioplene, R., Caprihan, A., Chavez, R. S., \& Haier, R. J. (2010). White matter integrity, creativity, and psychopathology: Disentangling constructs with diffusion tensor imaging. PLoS ONE, 5(3). https://doi.org/10.1371/journal.pone.0009818

Kajantie, E., \& Phillips, D. I. W. (2006). The effects of sex and hormonal status on the physiological response to acute psychosocial stress. Psychoneuroendocrinology, 31, 151178. https://doi.org/10.1016/j.psyneuen.2005.07.002

Kalia, M. (2002). Assessing the economic impact of stress - The modern day hidden epidemic. Metabolism: Clinical and Experimental, 51(6 SUPPL. 1), 49-53. https://doi.org/10.1053/meta.2002.33193

Karg, K., Burmeister, M., Shedden, K., \& Sen, S. (2011). The Serotonin Transporter Promoter Variant (5-HTTLPR), Stress, and Depression Meta-analysis Revisited. Archives of General Psychiatry, 68(5), 444-454. https://doi.org/10.1001/archgenpsychiatry.2010.189

Karimi, Z., Windmann, S., Güntürkün, O., \& Abraham, A. (2007). Insight problem solving in individuals with high versus low schizotypy. Journal of Research in Personality, 41(2), 473-480. https://doi.org/10.1016/j.jrp.2006.03.008 
Kelley, B. J., Yeager, K. R., Pepper, T. H., Bornstein, R. A., \& Beversdorf, D. Q. (2007). The Effect of Propranolol on Cognitive Flexibility and Memory in Acute Cocaine Withdrawal. Neurocase, 13, 320-327. https://doi.org/10.1080/13554790701846148

Kelley, B. J., Yeager, K. R., Pepper, T. H., \& David, Q. (2005). Cognitive Impairment in Acute Cocaine Withdrawal. Cognitive and Behavioral Neurology, 18(2), 108-112.

Kemeny, M. E. (2003). The Psychobiology of Stress. Current Directions in Psychological Science, 12(4), 124-129.

Kempton, M. J., Haldane, M., Jogia, J., Christodoulou, T., Powell, J., Collier, D., ... Frangou, S. (2009). The effects of gender and COMT Val158Met polymorphism on fearful facial affect recognition: A fMRI study. International Journal of Neuropsychopharmacology, 12(3), 371-381. https://doi.org/10.1017/S1461145708009395

Kenett, Y. N., Levy, O., Kenett, D. Y., Stanley, H. E., Faust, M., \& Havlin, S. (2018). Flexibility of thought in high creative individuals represented by percolation analysis. Proceedings of the National Academy of Sciences, 201717362. https://doi.org/10.1073/pnas.1717362115

Kenna, G. A., Roder-hanna, N., Leggio, L., Zywiak, W. H., Clifford, J., Edwards, S., ... Swift, R. M. (2012). Association of the 5-HTT gene-linked promoter region (5-HTTLPR ) polymorphism with psychiatric disorders : review of psychopathology and pharmacotherapy. Pharmacogenomics and Personalized Medicine, 5, 19-35.

Kessler, R. C. (2003). Epidemiology of women and depression. Journal of Affective Disorders, 74(1), 5-13. https://doi.org/10.1016/S0165-0327(02)00426-3 
Kessler, R. C., McGonagle, K. A., Swartz, M., Blazer, D. G., \& Nelson, C. B. (1993). Sex and depression in the National Comorbidity Survey I: Lifetime prevalence, chronicity and recurrence. Journal of Affective Disorders, 29(2-3), 85-96. https://doi.org/10.1016/0165-0327(93)90026-G

Khalili-Mahani, N., Dedovic, K., Engert, V., Pruessner, M., \& Pruessner, J. C. (2010). Hippocampal activation during a cognitive task is associated with subsequent neuroendocrine and cognitive responses to psychological stress. Hippocampus, 20(2), 323-334. https://doi.org/10.1002/hipo.20623

Kim, M. J., Loucks, R. A., Palmer, A. L., Brown, A. C., Solomon, K. M., Marchante, A. N., \& Whalen, P. J. (2011). The structural and functional connectivity of the amygdala: From normal emotion to pathological anxiety. Behavioural Brain Research, 223(2), 403410. https://doi.org/10.1016/j.bbr.2011.04.025

Kirschbaum, C., Pirke, K.-M., \& Hellhammer, D. (1993). The ' Trier Social Stress Test' - A Tool for Investigating Psychobiological Stress Responses in a Laboratory Setting. Neuropsychobiology, 28, 76-81.

Kirschbaum, C., Wust, S., \& Hellhammer, D. (1992). Consistent Sex Differences in Cortisol Responses to Psychological Stress. Psychosomatic Medicine, 54, 648-657.

Kitraki, E., Kremmyda, O., Youlatos, D., Alexis, M. N., \& Kittas, C. (2004). Genderdependent alterations in corticosteroid receptor status and spatial performance following 21 days of restraint stress. Neuroscience, 125, 47-55. https://doi.org/10.1016/j.neuroscience.2003.12.024

Klein, L. C., \& Corwin, E. J. (2002). Seeing the Unexpected : How Sex Differences in Stress Responses May Provide a New Perspective on the Manifestation of Psychiatric 
Disorders. Current Psychiatry Reports, 4, 441-448.

Kleinhans, N. M., Richards, T., Weaver, K., Johnson, L. C., Greenson, J., ... Aylward, E. (2010). Association between amygdala response to emotional faces and social anxiety in autism spectrum disorders. Neuropsychologia, 48(12), 3665-3670.

https://doi.org/10.1016/j.neuropsychologia.2010.07.022

Kliemann, D., Dziobek, I., Hatri, A., Baudewig, J., \& Heekeren, H. R. (2012). The Role of the Amygdala in Atypical Gaze on Emotional Faces in Autism Spectrum Disorders. Journal of Neuroscience, 32(28), 9469-9476. https://doi.org/10.1523/JNEUROSCI.529411.2012

Kobiella, A., Reimold, M., Ulshofer, D., Ikonomidou, V., Vollmert, C., Vollstadt-Klein, S., ... Smolka, M. (2011). How the serotonin transporter 5-HTTLPR polymorphism influences amygdala function : the roles of in vivo serotonin transporter expression and amygdala structure. Translational Psychiatry, e37(March), 1-9.

https://doi.org/10.1038/tp.2011.29

Kogler, L., Seidel, E. M., Metzler, H., Thaler, H., Boubela, R. N., Pruessner, J. C., ... Derntl, B. (2017). Impact of self-esteem and sex on stress reactions. Scientific Reports, 7(1), 1-9. https://doi.org/10.1038/s41598-017-17485-w

Koob, G. F., Cole, B. J., Swerdlow, N. R., Le Moal, M., \& Britton, K. T. (1990). Stress, performance, and arousal: Focus on CRF. NIDA Research Monograph, 97, 163-176.

Kruschwitz, J. D., Walter, M., Varikuti, D., Jensen, J., Plichta, M. M., Haddad, L., ... Walter, H. (2015). 5-HTTLPR/rs25531 polymorphism and neuroticism are linked by resting state functional connectivity of amygdala and fusiform gyrus. Brain Structure and Function, 220(4), 2373-2385. https://doi.org/10.1007/s00429-014-0782-0 
Kühn, S., Ritter, S. M., Müller, B. C. N., van Baaren, R. B., Brass, M., \& Dijksterhuis, A. (2014). The Importance of the Default Mode Network in Creativity-A Structural MRI Study. The Journal of Creative Behavior, 48(2), 152-163. https://doi.org/10.1002/jocb.45

Laverdure, B., \& Boulenger, J.-P. (1991). Medications beta-bloquantes et anxiete Un interest therapeutique certain. L'Encephale, XVII, 481-492.

LeDoux, J. (2007). The amygdala. Current Biology, 17(20), 868-874. https://doi.org/10.1016/j.cub.2007.08.005

Lesch, K.-P., Bengel, D., Heils, A., Sabol, S. Z., Greenberg, B. D., Petri, S., ... Murphy, D. L. (1996). Association of anxiety-related traits with a polymorphism in the serotonin transporter gene regulatory region. Science (New York, N.Y.), 274(5292), 1527-1531. Retrieved from http://www.ncbi.nlm.nih.gov/pubmed/8929413\%0Ahttp://www.jstor.org/stable/289226

Li, S., Weerda, R., Milde, C., Wolf, O. T., \& Thiel, C. M. (2015). ADRA2B genotype differentially modulates stress-induced neural activity in the amygdala and hippocampus during emotional memory retrieval. Psychopharmacology, 232(4), 755-764. https://doi.org/10.1007/s00213-014-3710-3

Libkuman, T. M., Otani, H., Kern, R., Viger, S. G., \& Novak, N. (2007). Multidimensional normative ratings for the International Affective Picture System. Behavior Research Methods, 39(2), 326-334. https://doi.org/10.3758/BF03193164

Lighthall, N. R., Sakaki, M., Vasunilashorn, S., Nga, L., Somayajula, S., Chen, E. Y., ... Mather, M. (2012). Gender differences in reward-related decision processing under stress. Social Cognitive and Affective Neuroscience, 7(4), 476-484. 
https://doi.org/10.1093/scan/nsr026

Limb, C. J., \& Braun, A. R. (2008). Neural substrates of spontaneous musical performance: An fMRI study of jazz improvisation. PLoS ONE, 3(2). https://doi.org/10.1371/journal.pone.0001679

Lipnicki, D. M., \& Byrne, D. G. (2005). Thinking on your back: Solving anagrams faster when supine than when standing. Cognitive Brain Research, 24(3), 719-722. https://doi.org/10.1016/j.cogbrainres.2005.03.003

Liston, C., Mcewen, B. S., \& Casey, B. J. (2009). Psychosocial stress reversibly disrupts prefrontal processing and attentional control. PNAS, 106(3), 912-917. https://doi.org/10.1073/pnas.0807041106

Lucassen, P. J., Pruessner, J., Sousa, N., Almeida, O. F. X., Marie, A., Rajkowska, G., ... Czéh, B. (2014). Neuropathology of stress. Acta Neuropathol, 127, 109-135. https://doi.org/10.1007/s00401-013-1223-5

Martindale, C., \& Greenough, J. (1973). The differential effect of increased arousal on creative and intellectual performance. J Genet Psychol, 123(2nd Ha, 329-335.

Mather, M., Lighthall, N. R., Nga, L., \& Gorlick, M. A. (2010). Sex differences in how stress affects brain activity during face viewing. NeuroReport, 21(14), 933-937. https://doi.org/10.1097/WNR.0b013e32833ddd92.Sex

Matud, M. P. (2004). Gender differences in stress and coping styles. Personality and Individual Differences, 37, 1401-1415. https://doi.org/10.1016/j.paid.2004.01.010 
Mcewen, B. S. (2009). The Brain is the Central Organ of Stress and Adaptation.

Neuroimage, 47(3), 911-913. https://doi.org/10.1016/j.neuroimage.2009.05.071.The

McEwen, B. S. (2007). Physiology and Neurobiology of Stress and Adaptation : Central Role of the Brain. Physiol Rev, 87, 873-904.

https://doi.org/10.1152/physrev.00041.2006.

McEwen, B. S., Nasca, C., \& Gray, J. D. (2016). Stress Effects on Neuronal Structure: Hippocampus, Amygdala, and Prefrontal Cortex. Neuropsychopharmacology, 41(1), 323. https://doi.org/10.1038/npp.2015.171

McEwen, B. S., \& Saplosky, R. M. (1995). Stress and Cognitive Function. Current Opinion in Neurobiology, 5, 205-216.

Mcguffin, P., Alsabban, S., \& Uher, R. (2011). The truth about genetic variation in the serotonin transporter gene and response to stress and medication. The British Journal of Psychiatry, 198, 424-427. https://doi.org/10.1192/bjp.bp.110.085225

Mihov, K. M., Denzler, M., \& Förster, J. (2010). Hemispheric specialization and creative thinking: A meta-analytic review of lateralization of creativity. Brain and Cognition, 72(3), 442-448. https://doi.org/10.1016/j.bandc.2009.12.007

Ming, Q., Zhang, Y., Yi, J., Wang, X., Zhu, X., \& Yao, S. (2015). Serotonin transporter gene polymorphism ( 5-HTTLPR ) L allele interacts with stress to increase anxiety symptoms in Chinese adolescents : a multiwave longitudinal study. BMC Psychiatry, 15, 248-255. https://doi.org/10.1186/s12888-015-0639-y

Monk, C. S., \& Telzer, E. H. (2008). Amygdala and ventrolateral prefrontal cortex activation to masked angry faces in children and adolescents with generalized anxiety 
disorder. Arch. Gen. Psychiatry, 65(5), 568-576.

Morris, J. S., Friston, K. J., Büchel, C., Frith, C. D., Young, A. W., Calder, A. J., \& Dolan, R. J. (1998). A neuromodulatory role for the human amygdala in processing emotional facial expressions. Brain: A Journal of Neurology, 121 ( Pt 1, 47-57. https://doi.org/10.1093/brain/121.1.47

Mueller, A., Armbruster, D., Moser, D. A., Canli, T., Lesch, K., Brocke, B., \& Kirschbaum, C. (2011). Interaction of Serotonin Transporter Gene-Linked Polymorphic Region and Stressful Life Events Predicts Cortisol Stress Response. Neuropsychopharmacology, 36(7), 1332-1339. https://doi.org/10.1038/npp.2011.11

Murphy, D. L., \& Moya, P. R. (2011). Human Serotonin Transporter Gene (SLC6A4) Variants: Their Contributions to Understanding Pharmacogenomic and Other Functional G x G and G x E Differences in Health and Disease. Current Opinion in Pharmacology, 11(1), 3-10. https://doi.org/10.1016/j.coph.2011.02.008.Human

Narayanan, A., White, C. A., Saklayen, S. S., Abduljalil, A., Schmalbrock, P., Pepper, T. H., ... Beversdorf, D. Q. (2012). Functional connectivity during language processing in acute cocaine withdrawal : A pilot study. Neurocase, 18(6), 441-449.

Narayanan, A., White, C. A., Saklayen, S., Scaduto, M. J., Carpenter, A. L., Abduljalil, A., ... Beversdorf, D. Q. (2010). Effect of propranolol on functional connectivity in autism spectrum disorder-A pilot study. Brain Imaging and Behavior, 4(2), 189-197. https://doi.org/10.1007/s11682-010-9098-8

Nesse, R. M., Bhatnagar, S., \& Ellis, B. (2016). Evolutionary Origins and Functions of the Stress Response System. Stress: Concepts, Cognition, Emotion, and Behavior: Handbook of Stress, 1, 95-101. https://doi.org/10.1016/B978-0-12-800951-2.00011-X 
Neubauer, A. C., \& Fink, A. (2009). Intelligence and neural efficiency. Neuroscience and Biobehavioral Reviews, 33(7), 1004-1023.

https://doi.org/10.1016/j.neubiorev.2009.04.001

Oei, N. Y. L., Veer, I. M., Wolf, O. T., Spinhoven, P., Rombouts, S. A. R. B., \& Elzinga, B. M. (2012). Stress shifts brain activation towards ventral' affective' areas during emotional distraction. SCAN, 7, 403-412. https://doi.org/10.1093/scan/nsr024

Onur, O. A., Walter, H., Schlaepfer, T. E., Rehme, A. K., Schmidt, C., Keysers, C., ... Hurlemann, R. (2009). Noradrenergic enhancement of amygdala responses to fear. Social Cognitive and Affective Neuroscience, 4(2), 119-126.

https://doi.org/10.1093/scan/nsn049

Ossewaarde, L., Qin, S., Van Marle, H. J. F., van Wingen, G. A., Fernández, G., \& Hermans, E. J. (2011). Stress-induced reduction in reward-related prefrontal cortex function. NeuroImage, 55(1), 345-352. https://doi.org/10.1016/j.neuroimage.2010.11.068

Pechtel, P., \& Pizzagalli, D. A. (2011). Effects of early life stress on cognitive and affective function : an integrated review of human literature. Psychopharmacology, 214(1), 55-70. https://doi.org/10.1007/s00213-010-2009-2

Pezawas, L., Meyer-lindenberg, A., Drabant, E. M., Verchinski, B. A., Munoz, K. E., Kolachana, B. S., ... Weinberger, D. R. (2005). 5-HTTLPR polymorphism impacts human cingulate- amygdala interactions : a genetic susceptibility mechanism for depression. Nature Neuroscience, 8(6), 828-834. https://doi.org/10.1038/nn1463

Pezawas, L., Meyer-Lindenberg, A., Drabant, E. M., Verchinski, B. A., Munoz, K. E., Kolachana, B. S., ... Weinberger, D. R. (2005). 5-HTTLPR polymorphism impacts 
human cingulate-amygdala interactions: A genetic susceptibility mechanism for depression. Nature Neuroscience, 8(6), 828-834. https://doi.org/10.1038/nn1463

Pruessner, J. C., Dedovic, K., Khalili-mahani, N., Engert, V., Pruessner, M., Buss, C., ... Lupien, S. (2008). Deactivation of the Limbic System During Acute Psychosocial Stress : Evidence from Positron Emission Tomography and Functional Magnetic Resonance Imaging Studies. Biol Psychiatry, 63, 234-240. https://doi.org/10.1016/j.biopsych.2007.04.041

Pruessner, J. C., Dedovic, K., Pruessner, M., Lord, C., Buss, C., Collins, L., ... Lupien, S. J. (2010). Stress regulation in the central nervous system: evidence from structural and functional neuroimaging studies in human populations - 2008 Curt Richter Award Winner. Psychoneuroendocrinology, 35(1), 179-191.

https://doi.org/10.1016/j.psyneuen.2009.02.016

Pruessner, M., Pruessner, J. C., Hellhammer, D. H., Bruce Pike, G., \& Lupien, S. J. (2007). The associations among hippocampal volume, cortisol reactivity, and memory performance in healthy young men. Psychiatry Research - Neuroimaging, 155(1), 1-10. https://doi.org/10.1016/j.pscychresns.2006.12.007

Qin, S., Cousijn, H., Rijpkema, M., Luo, J., Franke, B., Hermans, E. J., \& Fernández, G. (2012). The effect of moderate acute psychological stress on working memory-related neural activity is modulated by a genetic variation in catecholaminergic function in humans. Frontiers in Integrative Neuroscience, 6(May), 1-12. https://doi.org/10.3389/fnint.2012.00016

Qin, S., Hermans, E. J., van Marle, H. J. F., \& Fernandez, G. (2012). Understanding Low Reliability of Memories for Neutral Information Encoded under Stress: Alterations in Memory-Related Activation in the Hippocampus and Midbrain. Journal of Neuroscience, 
32(12), 4032-4041. https://doi.org/10.1523/JNEUROSCI.3101-11.2012

Qin, S., Hermans, E. J., van Marle, H. J. F., Luo, J., \& Fernández, G. (2009). Acute Psychological Stress Reduces Working Memory-Related Activity in the Dorsolateral Prefrontal Cortex. Biological Psychiatry, 66(1), 25-32. https://doi.org/10.1016/j.biopsych.2009.03.006

Quaedflieg, C. W. E. M., Van De Ven, V., Meyer, T., Siep, N., Merckelbach, H., \& Smeets, T. (2015). Temporal dynamics of stress-induced alternations of intrinsic amygdala connectivity and neuroendocrine levels. PLoS ONE, 10(5), 1-16. https://doi.org/10.1371/journal.pone.0124141

Rabinak, C. A., Angstadt, M., Welsh, R. C., Kenndy, A. E., Lyubkin, M., Martis, B., \& Luan Phan, K. (2011). Altered amygdala resting-state functional connectivity in posttraumatic stress disorder. Frontiers in Psychiatry, 2(NOV), 1-8. https://doi.org/10.3389/fpsyt.2011.00062

Rauch, S. L., Whalen, P. J., Shin, L. M., McInerney, S. C., Macklin, M. L., Lasko, N. B., ... Pitman, R. K. (2000). Exaggerated amygdala response to masked facial stimuli in posttraumatic stress disorder: a functional MRI study. Biol.Psychiatry, 47(9), 769-776. https://doi.org/10.1016/S0006-3223(00)00828-3

Renner, K. H., \& Beversdorf, D. Q. (2010). Effects of naturalistic stressors on cognitive flexibility and working memory task performance. Neurocase, 16(4), 293-300. https://doi.org/10.1080/13554790903463601

Reuter, M., Roth, S., Holve, K., \& Hennig, J. (2006). Identification of first candidate genes for creativity: A pilot study. Brain Research, 1069(1), 190-197.

https://doi.org/10.1016/j.brainres.2005.11.046 
Risch, N., Herrell, R., Lehner, T., Liang, K.-Y., Eaves, L., Hoh, J., ... Merikangas, K. R. (2009). Interaction Between the Serotonin Transporter Gene (5-HTTLPR), Stressful Life Events, and Risk of Depression. JAMA, 301(23), 2462-2471.

Rodríguez-Aranda, C., \& Martinussen, M. (2006). Age-related differences in performance of phonemic verbal fluency measured by Controlled Oral Word Association Task (COWAT): A meta-analytic study. Developmental Neuropsychology, 30(2), 697717. https://doi.org/10.1207/s15326942dn3002_3

Roy, A. K., Shehzad, Z., Margulies, D. S., Kelly, A. M. C., Uddin, Q. L., Gotimer, K., ... Milham, M. P. (2010). Functional connectivity of the human amygdala using resting state fMRI. NeuroImage, 45(2), 614-626.

https://doi.org/10.1016/j.neuroimage.2008.11.030.Functional

Rudolph, K. D., \& Hammen, C. (1999). Age and Gender as Determinants of Stress Exposure, Generation, and Reactions in Youngsters : A Transactional Perspective Author ( s ): Karen D . Rudolph and Constance Hammen Published by: Wiley on behalf of the Society for Research in Child Development. Child Development, 70(3), 660-677. Sandi, C. (2013). Stress and cognition. WIREs Cogn Sci, 4, 245-261. https://doi.org/10.1002/wcs.1222

Sapolsky, R. M., Krey, L. C., \& McEwen, B. S. (1986). The Neuroendocrinology of Stress and Aging: The Glucocorticoid Cascade Hypothesis. Endocrine Reviews, 7(3), 284-301. https://doi.org/10.1210/edrv-7-3-284

Schultz, R. T. (2005). Developmental deficits in social perception in autism: The role of the amygdala and fusiform face area. International Journal of Developmental Neuroscience, 23(2-3 SPEC. ISS.), 125-141. 
https://doi.org/10.1016/j.ijdevneu.2004.12.012

Shields, G. S., Sazma, M. A., \& Yonelinas, A. P. (2016). The effects of acute stress on core executive functions: A meta-analysis and comparison with cortisol. Neuroscience and Biobehavioral Reviews, 68, 651-668.

https://doi.org/10.1016/j.neubiorev.2016.06.038

Smith, S. M. (2002). Fast robust automated brain extraction. Human Brain Mapping, 17(3), 143-155. https://doi.org/10.1002/hbm.10062

Smolka, M. N. (2005). Catechol-O-Methyltransferase val158met Genotype Affects Processing of Emotional Stimuli in the Amygdala and Prefrontal Cortex. Journal of Neuroscience, 25(4), 836-842. https://doi.org/10.1523/JNEUROSCI.1792-04.2005

Soares, J. M., Sampaio, A., Ferreira, L. M., Santos, N. C., Marques, P., Marques, F., ... Sousa, N. (2013). Stress Impact on Resting State Brain Networks. PLoS ONE, 8(6), 1-9. https://doi.org/10.1371/journal.pone.0066500

Sripada, R. K., King, A. P., Garfinkel, S. N., Wang, X., Sripada, C. S., Welsh, R. C., \& Liberzon, I. (2012). Altered resting-state amygdala functional connectivity in men with posttraumatic stress disorder. Journal of Psychiatry and Neuroscience, 37(4), 241-249. https://doi.org/10.1503/jpn.110069

Stevens, J. S., Jovanovic, T., Fani, N., Ely, T. D., Glover, E. M., Bradley, B., \& Ressler, K. J. (2013). Disrupted amygdala-prefrontal functional connectivity in civilian women with posttraumatic stress disorder. Journal of Psychiatric Research, 47(10), 1469-1478. https://doi.org/10.1016/j.jpsychires.2013.05.031

Strauss, E., Sherman, E. M., \& Spreen, O. (1998). A compendium of neuropsychological 
tests: Administration, norms, and commentary. Oxford University Press, Oxford Ed.

Sweeten, T. L., Posey, D. J., Shekhar, A., \& McDougle, C. J. (2002). The amygdala and related structures in the pathophysiology of autism. Pharmacology Biochemistry and Behavior, 71(3), 449-455. https://doi.org/10.1016/S0091-3057(01)00697-9

ter Horst, J. P., de Kloet, E. R., Schachinger, H., \& Oitzl, M. S. (2012). Relevance of Stress and Female Sex Hormones for Emotion and Cognition. Cell Mol Neurobiol, 32, 725-735. https://doi.org/10.1007/s10571-011-9774-2

Tivarus, M. E., Hillier, A., Schmalbrock, P., \& Beversdorf, D. Q. (2008). Functional connectivity in an fMRI study of semantic and phonological processes and the effect of $\mathrm{L}$ -Dopa. Brain and Language, 104(1), 42-50. https://doi.org/10.1016/j.bandl.2007.02.007

Toffoletto, S., Lanzenberger, R., Gingnell, M., Sundström-poromaa, I., \& Comasco, E. (2014). Emotional and cognitive functional imaging of estrogen and progesterone effects in the female human brain: A systematic review. Psychoneuroendocrinology, 50, 28-52. https://doi.org/10.1016/j.psyneuen.2014.07.025

Tomasi, D., \& Volkow, N. D. (2012). Resting functional connectivity of language networks: Characterization and reproducibility. Molecular Psychiatry, 17(8), 841-854. https://doi.org/10.1038/mp.2011.177

Uttl, B. (2002). North American Adult Reading Test: Age Norms, Reliability, and Validity. Journal of Clinical and Experimental Neuropsychology, 24(8), 1123-1137.

Vaisvaser, S., Lin, T., Admon, R., Podlipsky, I., Greenman, Y., Stern, N., ... Hendler, T. (2013). Neural traces of stress: cortisol related sustained enhancement of amygdalahippocampal functional connectivity. Frontiers in Human Neuroscience, 7(July), 1-11. 
https://doi.org/10.3389/fnhum.2013.00313

Vamvakopoulos, N. C., \& Chrousos, G. P. (1993). Evidence of direct estrogenic regulation of human corticotropin-releasing hormone gene expression. Potential implications for the sexual dimophism of the stress response and immune / inflammatory reaction . Find the latest version: The Journal of Clinical Investigation, 92(4), 18961902.

Vamvakopoulos, N. C., \& Chrousos, G. P. (1994). Hormonal Regulation of Human Corticotropin-Releasing Hormone Gene Expression : Implications for the Stress Response and Immune / Inflammatory Reaction. Endocrine Reviews, 15(4), 409-420.

van der Meer, D., Hartman, C. A., Pruim, R. H. R. R., Mennes, M., Heslenfeld, D., Oosterlaan, J., ... Hoekstra, P. J. (2016). The interaction between 5-HTTLPR and stress exposure influences connectivity of the executive control and default mode brain networks. Brain Imaging and Behavior, 11(5), 1-11. https://doi.org/10.1007/s11682-0169633-3

van der Meer, D., Hartman, C. A., Richards, J., Bralten, J. B., Franke, B., Oosterlaan, J., ... Hoekstra, P. J. (2014). The serotonin transporter gene polymorphism 5-HTTLPR moderates the effects of stress on attention-deficit / hyperactivity disorder. The Journal of Child Psychology and Psychiatry, 55(12), 1363-1371. https://doi.org/10.1111/jcpp.12240

Van Der Meer, D., Hoekstra, P. J., Zwiers, M., Mennes, M., Schweren, L. J., Franke, B., ... Hartman, C. A. (2015). Brain correlates of the interaction between 5-HTTLPR and psychosocial stress mediating attention deficit hyperactivity disorder severity. American Journal of Psychiatry, 172(8), 768-775. https://doi.org/10.1176/appi.ajp.2015.14081035

van Marle, H. J. F., Hermans, E. J., Qin, S., \& Fernández, G. (2009). From Specificity to 
Sensitivity: How Acute Stress Affects Amygdala Processing of Biologically Salient Stimuli. Biological Psychiatry, 66(7), 649-655.

https://doi.org/10.1016/j.biopsych.2009.05.014

van Marle, H. J. F., Hermans, E. J., Qin, S., \& Fernández, G. (2010). Enhanced restingstate connectivity of amygdala in the immediate aftermath of acute psychological stress. NeuroImage, 53(1), 348-354. https://doi.org/10.1016/j.neuroimage.2010.05.070

Van Stegeren, A. H., Goekoop, R., Everaerd, W., Scheltens, P., Barkhof, F., Kuijer, J. P. A., \& Rombouts, S. A. R. B. (2005). Noradrenaline mediates amygdala activation in men and women during encoding of emotional material. NeuroImage, 24(3), 898-909. https://doi.org/10.1016/j.neuroimage.2004.09.011

Veer, I. M., Oei, N. Y. L., Spinhoven, P., van Buchem, M. A., Elzinga, B. M., \& Rombouts, S. A. R. B. (2011). Beyond acute social stress: Increased functional connectivity between amygdala and cortical midline structures. NeuroImage, 57(4), 1534-1541. https://doi.org/10.1016/j.neuroimage.2011.05.074

Veer, I. M., Oei, N. Y. L., Spinhoven, P., Van Buchem, M. A., Elzinga, B. M., \& Rombouts, S. A. R. B. (2012). Endogenous cortisol is associated with functional connectivity between the amygdala and medial prefrontal cortex. Psychoneuroendocrinology, 37(7), 1039-1047. https://doi.org/10.1016/j.psyneuen.2011.12.001

Volman, I., Verhagen, L., den Ouden, H. E. M., Fernandez, G., Rijpkema, M., Franke, B., ... Roelofs, K. (2013). Reduced Serotonin Transporter Availability Decreases Prefrontal Control of the Amygdala. Journal of Neuroscience, 33(21), 8974-8979. https://doi.org/10.1523/JNEUROSCI.5518-12.2013 
Vuilleumier, P., Richardson, M. P., Armony, J. L., Driver, J., \& Dolan, R. J. (2004).

Distant influences of amygdala lesion on visual cortical activation during emotional face processing. Nature Neuroscience, 7(11), 1271-1278. https://doi.org/10.1038/nn1341

Wang, J., Korczykowski, M., Rao, H., Fan, Y., Pluta, J., Gur, R. C., ... Detre, J. A. (2007). Gender difference in neural response to psychological stress. SCAN, 2(3), 227239. https://doi.org/10.1093/scan/nsm018

Weis, S., Hausmann, M., Stoffers, B., \& Sturm, W. (2011). Dynamic changes in functional cerebral connectivity of spatial cognition during the menstrual cycle. Human Brain Mapping, 32(10), 1544-1556. https://doi.org/10.1002/hbm.21126

Weiss, E. M., Hofer, A., Golaszewski, S., Siedentopf, C., Brinkhoff, C., Kremser, C., ... Fleischhacker, W. W. (2004). Brain activation patterns during a verbal fluency test - a functional MRI study in healthy volunteers and patients with schizophrenia.

Schizophrenia Research, 70, 287-291. https://doi.org/10.1016/j.schres.2004.01.010

Weissman, M. M., \& Klerman, G. L. (1977). Sex Differences and the Epidemiology of Depression. Archives of General Psychiatry, 34(1), 98-111. https://doi.org/10.1001/archpsyc.1977.01770130100011

Whalen, P. J., Shin, L. M., McInerney, S. C., Fischer, H., Wright, C. I., \& Rauch, S. L. (2001). A Functional MRI Study of Human Amygdala Responses to Facial Expressions of Fear Versus Anger. Emotion, 1(1), 70-83. https://doi.org/10.1037/1528-3542.1.1.70

Williams, L. M., Das, P., Harris, A. W. F., Liddell, B. B., Brammer, M. J., Olivieri, G., ... Gordon, E. (2004). Dysregulation of arousal and amygdala-prefrontal systems in paranoid schizophrenia. The American Journal of Psychiatry, 161(3), 480-489. https://doi.org/10.1176/appi.ajp.161.3.480 
Worsley, K. J. (2001). Statistical analysis of activation images. In Functional MRI: An Introduction to Methods, Oxford University Press (p. Chapter 14).

Wrase, J., Klein, S., Gruesser, S. M., Hermann, D., Flor, H., Mann, K., ... Heinz, A. (2003). Gender differences in the processing of standardized emotional visual stimuli in humans: A functional magnetic resonance imaging study. Neuroscience Letters, 348(1), 41-45. https://doi.org/10.1016/S0304-3940(03)00565-2

Zhao, Q., Zhou, Z., Xu, H., Fan, W., \& Han, L. (2014). Neural pathway in the right hemisphere underlies verbal insight problem solving. Neuroscience, 256, 334-341. https://doi.org/10.1016/j.neuroscience.2013.10.019 


\section{VITA}

Neetu Nair was born in New Delhi, India and grew up in Kochi, Kerala, India. She did most of her schooling at Bhavan's Adarsha Vidyalaya, Kochi, Kerala, India. She graduated with a Bachelor of Technology degree in Electronics and Biomedical Engineering from Model Engineering College, Cochin University of Science and Technology, Kochi, Kerala, India, in 2008. She started graduate school in the Neuroscience program at West Virginia University, Morgantown, West Virginia in 2011. In 2015, she transferred into the Interdisciplinary Neuroscience Program at the University of Missouri, Columbia, Missouri to complete her degree in Doctor of Philosophy. Upon completing her degree, Neetu will take up a post-doctoral fellowship position and continue research into novel and effective neuroimaging methods, especially, treatment prediction biomarkers, for neuropsychiatric conditions. 